\title{
Molecular mechanisms of substrate selection and protein folding on the ribosome
}

\author{
Dissertation \\ for the award of the degree \\ "Doctor rerum naturalium" (Dr. rer. nat.) \\ Division of Mathematics and Natural Sciences of \\ the Georg-August-Universität Göttingen
}

\author{
Submitted by \\ Jörg Mittelstät \\ from Oberhausen, Germany
}

Göttingen 2012 
Members of the Thesis Committee:

Prof. Dr. Marina V. Rodnina ( $1^{\text {st }}$ reviewer $)$

Department of Physical Biochemistry

Max Planck Institute for Biophysical Chemistry

Prof. Dr. Holger Stark ( $2^{\text {nd }}$ reviewer $)$

3D-Cryo Electron Microscopy

Max Planck Institute for Biophysical Chemistry

Prof. Dr. Ralf Ficner

Department of Molecular Structural Biology

Göttingen Center for Molecular Biosciences

Date of oral examination: June $19^{\text {th }}, 2012$ 


\section{Affidavit}

I hereby declare that I prepared the dissertation "Molecular mechanisms of substrate selection and protein folding on the ribosome" on my own and with no other sources and aids than quoted.

Jörg Mittelstät

Göttingen, May $21^{\text {st }}, 2012$ 


\section{TABLE OF CONTENTS}

SUMMARY 1

$\begin{array}{ll}\text { INTRODUCTION } & 2\end{array}$

1. Protein synthesis in living cells 2

1.1 The four main phases of protein biosynthesis on the ribosome 3

2. The fidelity of protein biosynthesis 5

2.1 Fidelity of aminoacylation and aa-tRNA binding to EF-Tu 6

2.2 Fidelity mechanisms on the ribosome 7

2.3 Bypassing of translational fidelity mechanisms to incorporate $\begin{array}{ll}\text { non-canonical amino acids into proteins } & 15\end{array}$

3. Non-uniform rates of protein synthesis 19

4. Co-translational protein folding 20

AIMS OF THE THESIS $\quad 24$

I DISTORTION OF tRNA UPON NEAR-COGNATE CODON RECOGNITION ON THE RIBOSOME 25

II KINETIC ANALYSIS OF THE INCORPORATION OF A FLUORESCENT NON-CANONICAL AMINO ACID ON THE $\begin{array}{ll}\text { RIBOSOME } & 40\end{array}$

III ANALYSIS OF NON-UNIFORM ELONGATION KINETICS DURING mRNA TRANSLATION BY SINGLE RIBOSOMES 60

IV REAL-TIME CO-TRANSLATIONAL FOLDING OF THE N-TERMINAL DOMAIN OF PROTEIN $\begin{array}{ll}\text { METHYLTRANSFERASE PrmC } & 76\end{array}$

REFERENCES $\quad 86$

$\begin{array}{ll}\text { ACKNOWLEDGEMENTS } & 102\end{array}$

$\begin{array}{ll}\text { CURRICULUM VITAE } & 103\end{array}$ 


\section{SUMMARY}

Aminoacyl-tRNA (aa-tRNA) is delivered to the ribosome in a ternary complex with elongation factor Tu (EF-Tu) and GTP. Cognate codon-anticodon interaction induces rapid GTP hydrolysis and allows aa-tRNA to accommodate on the ribosome and take part in peptide bond formation. To investigate how conformational changes in the aa-tRNA contribute to the induced-fit mechanism of aa-tRNA selection, we monitored the timing and extent of transient tRNA distortions upon reading cognate or near-cognate codons. Despite rather different GTP hydrolysis rates, the rearrangements are similar on cognate and near-cognate codons, suggesting that the tRNA distortion alone does not provide a specific switch for the preferential activation of GTP hydrolysis in EF-Tu on the cognate codon. While selection of canonical cognate aa-tRNAs on the ribosome is rapid and efficient, recruitment of tRNAs carrying non-canonical amino acids can be disfavored at several stages. Binding of $\varepsilon_{N_{2}}$-Bodipy576/589-Lys-tRNA ${ }^{\text {Lys }}$ to EF-Tu is strongly affected by the presence of the modified amino acid, but its incorporation on the ribosome occurs with speed and efficiency comparable to that of canonical lysine. The modified aatRNA is not significantly delayed at the accommodation gate and peptide bond formation is not rate-limiting for the incorporation, suggesting that ternary complex formation is the major bottleneck for the incorporation of non-canonical amino acids into proteins. Nonuniform translation elongation kinetics can affect many co-translational processes including protein folding. By pre-steady-state kinetic analysis of translation elongation on natural mRNAs, we find that translation is transiently paused at distinct sites, many of which (albeit not all) seem to be associated with rare codon clusters. By analysis of formation and decay kinetics of nascent peptides we find that chain elongation proceeds in a stochastic rather than sequential manner, which might provide a source of gene-intrinsic noise during protein expression. Co-translational protein folding is a general mechanism to prevent misfolding and aggregation of newly synthesized proteins. Here the cotranslational folding of the $\mathrm{N}$-terminal domain of protein PrmC was analyzed in real time by monitoring FRET between two fluorescently labeled amino acids incorporated at distinct positions of the polypeptide. The results indicate that folding of the N-terminal domain is an early event in the co-translational protein folding pathway, which is independent of the folding of the C-terminal domain. 


\section{INTRODUCTION ${ }^{1}$}

\section{Protein synthesis in living cells}

Proteins take essential functions in virtually every cellular process. Every protein is composed of an individual sequence of amino acids, which is encoded in the genetic code. The information necessary for the synthesis of a protein is extracted in two steps. First, the genetic code (DNA) is transcribed into messenger RNA (mRNA) by RNA polymerase, and then the mRNA is translated into proteins by ribosomes. The high fidelity and processivity during each step of protein biosynthesis are therefore critical parameters for the fitness of living cells. Ribosomes are large macromolecular machines composed of two subunits (Figure 1). In prokaryotes the $70 \mathrm{~S}$ ribosome has a mass of approximately $2.5 \mathrm{MDa}$ and is composed of a small $30 \mathrm{~S}$ and a large $50 \mathrm{~S}$ subunit. The small subunit consists of $16 \mathrm{~S}$ ribosomal RNA (rRNA) and 21 proteins and harbors the mRNA decoding center. Here mRNA codons are recognized based on base-pairing interactions with the anticodon of transfer RNAs (tRNAs) esterified to a specific amino acid at their acceptor end.

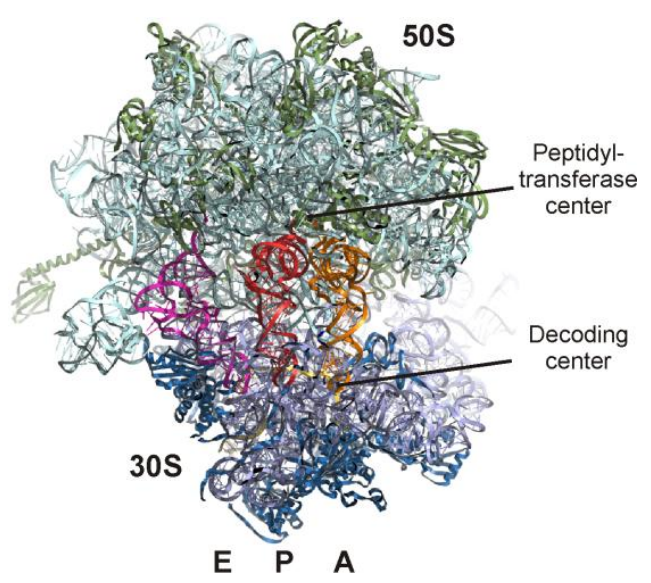

Figure 1 The 70S ribosome with three tRNAs and mRNA ((Jenner et al, 2010b), PDB codes: 3I8F and 3I8G). A, P, and E site tRNAs are shown in orange, red, and magenta, respectively. Messenger RNA is shown in yellow. 23S and 5S rRNA are palegreen, and 16S rRNA is palecyan. 50S and 30S ribosomal proteins are indicated in green and blue, respectively.

The large subunit is composed of $23 \mathrm{~S}$ and $5 \mathrm{~S}$ rRNA and 31 proteins. Its main function is the catalysis of peptide bond formation in the peptidyltransferase center. Three binding sites for tRNA have been identified on the ribosome, the aminoacyl-tRNA binding site (A site), the peptidyl-tRNA binding site (P site) and a binding site for deacylated tRNA called

\footnotetext{
${ }^{1}$ Parts of this review are published in the following review article: Wohlgemuth I, Pohl C, Mittelstaet J, Konevega AL, Rodnina MV (2011) Evolutionary optimization of speed and accuracy of decoding on the ribosome. Philos Trans R Soc Lond B Biol Sci 366: 2979-2986
} 
the exit site (E site). Structures derived from X-ray crystallography have provided insights into the atomic details of the ribosome structure (reviewed in (Bashan \& Yonath, 2008; Schmeing \& Ramakrishnan, 2009; Steitz, 2008)) and have supported the view that the ribosome is a ribozyme in which RNA not only serves as a structural framework but also acts as the catalyst for peptide bond formation (Cech, 2000; Nissen et al, 2000).

The central function of adaptor molecules, connecting the genetic code to the sequence of amino acids defining the primary structure of proteins, is performed by tRNAs. A tRNA molecule is composed of 73-93 nucleotides. Specific base pair interactions define the tRNA secondary structure comprising the acceptor arm, the D arm, the anticodon arm, the variable arm, and the TYC arm. Together, base pairing and base stacking interactions are responsible for the formation of the overall compact tertiary structure of the molecule which has a characteristic L-shape. tRNA bases are often specifically modified (Agris, 2004), which can be important for stabilizing tertiary structure elements but can also have an impact on the decoding properties and the interaction with proteins, such as aminoacyltRNA synthetases (aaRSs).

\subsection{The four main phases of protein biosynthesis on the ribosome}

On the ribosome protein synthesis proceeds in four main phases: Initiation, elongation, termination, and recycling. The fast and accurate progression through each of these steps requires the assistance of protein factors, many of them being GTPases (Figure 2). During the first step of translation initiation, the $30 \mathrm{~S}$ subunit associates with the initiation factors (IFs), mRNA, and initiator tRNA (fMet-tRNA ${ }^{\text {fMet }}$ ) to form the $30 \mathrm{~S}$ pre-initiation complex (Milon \& Rodnina, 2012). Selection of the mRNA is influenced by the secondary structure around its translation intiation region and is often guided by the interaction of the ShineDalgarno sequence 8-10 nt upstream of the initiation codon with the anti-Shine-Dalgarno sequence at the 3'end of 16S rRNA (Shine \& Dalgarno, 1974; Studer \& Joseph, 2006; Yusupova et al, 2006). This is followed by the recognition of the initiator codon by fMet$\mathrm{tRNA}^{\mathrm{fMet}}$ in the $\mathrm{P}$ site resulting in the formation of the $30 \mathrm{~S}$ initiation complex (Julian et al, 2011; Milon et al, 2012). Subsequently, the 50S subunit joins the 30S initiation complex, which triggers GTP hydrolysis in IF2 and the release of IF1 and IF3 (Milon et al, 2008; Tomsic et al, 2000). Dissociation of IF2 leads to the formation of the $70 \mathrm{~S}$ initiation complex which is ready for entering translation elongation.

Translation elongation is a cyclic process which requires two GTPases, elongation factors $\mathrm{Tu}$ and $\mathrm{G}$. In the first step of elongation, aminoacyl-tRNA (aa-tRNA) is delivered 
to the A site of the ribosome in a tight complex with EF-Tu and GTP. Codon-anticodon interaction takes place, GTP is hydrolyzed and EF-Tu loses its affinity for aa-tRNA, allowing the accommodation of aa-tRNA on the 50S subunit. Subsequent peptide bond formation transfers the peptide chain from the P site-bound peptidyl-tRNA (pept-tRNA) to the aa-tRNA in the A site. This results in peptide chain elongation by one amino acid, pepttRNA in the A site, and deacylated tRNA in the P site. This is followed by translocation, the movement of the ribosome by one codon triplet relative to the mRNA and the tRNAs, which is catalyzed by EF-G. EF-G in complex with GTP binds to the ribosome and hydrolyzes GTP, accelerating productive conformational rearrangements in the ribosome that ultimately lead to translocation (Peske et al, 2004; Rodnina et al, 1997; Savelsbergh et al, 2003). During translocation pept-tRNA moves from the A to the $\mathrm{P}$ site and deacylated tRNA is displaced from the $\mathrm{P}$ to the $\mathrm{E}$ site, resulting in a new mRNA codon presented in the vacant A site after EF-G has dissociated.

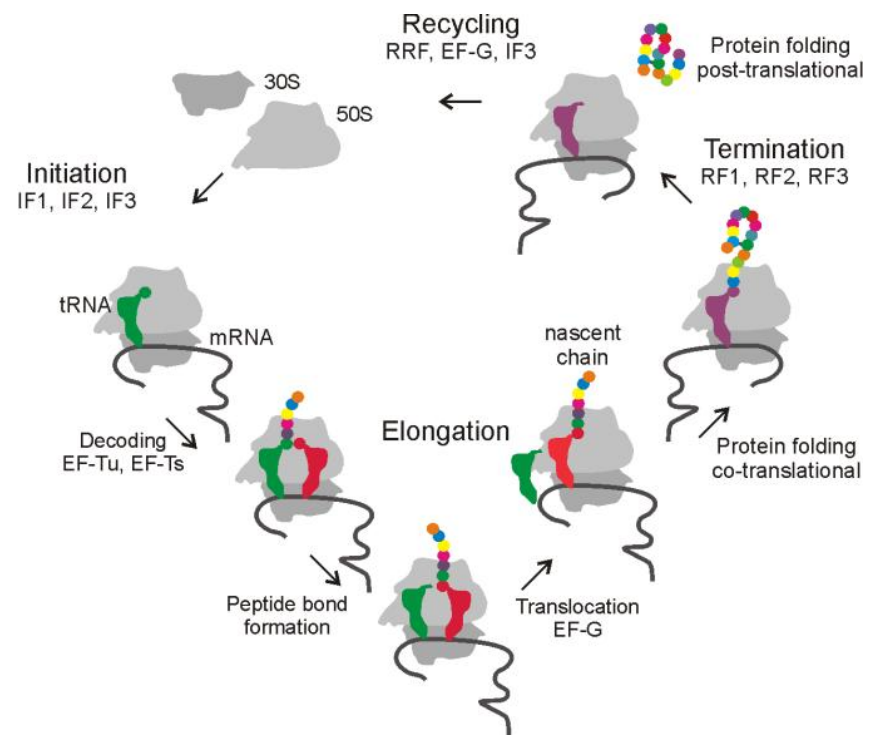

Figure 2 The main phases of translation. During initiation, the 50S and 30S subunit, together with initiator tRNA, mRNA, and with the help of initiation factors (IFs), form the 70S initiation complex. The 70S initiation complex then enters the elongation cycle during which aa-tRNA is delivered to the A site of the ribosome in ternary complex with EF-Tu and GTP. After accommodation of the aa-tRNA, peptide bond formation takes place. EF-G in complex with GTP then catalyzes the translocation reaction. During continued elongation, some proteins may already start to fold on the ribosome, while others fold posttranslationally. Once a stop codon is presented in the A site, elongation is terminated with the help of release factors (RFs). Finally, ribosome recycling leads to dissociation of the ribosomal subunits, deacylated tRNA, and mRNA.

During cyclic elongation the nascent protein chain is gradually increasing in length and traverses through the 50S ribosomal tunnel which is about $100 \AA$ long and $10-20 \AA$ wide (Ban et al, 2000). While small proteins fold post-translationally, due to the restrictions of the ribosomal tunnel which can accommodate peptides of about 30 aa length, larger 
multidomain proteins may undergo sequential folding events while they are being synthesized on the ribosome (Hartl et al, 2011).

Once a stop codon is presented in the A site, RF1 or RF2 bind to the ribosome and trigger hydrolytic cleavage of the peptide chain from pept-tRNA. In bacteria both factors recognize the UAA stop codon, while UAG is only recognized by RF1 and UGA only by RF2. This codon specificity is defined by a distinct tripeptide sequence in both factors (Ito et al, 2000). Another tripeptide sequence, the GGQ motif, which is post-translationally modified by methylation (Dincbas-Renqvist et al, 2000) is responsible for positioning the hydrolytic water molecule in the active site of the ribosome and essential for the catalytic activity of both release factors (Laurberg et al, 2008; Shaw \& Green, 2007; Weixlbaumer et al, 2008). After cleavage of the peptide chain, a third release factor RF3, which is a GTPase, binds to the ribosome and in a process that requires GTP hydrolysis, stimulates the dissociation of RF1/RF2 from the complex. The resulting posttermination complex the 70S ribosome with deacylated tRNA in the P site - is disassembled to allow for a new round of initiation. This recycling process requires the action of the ribosome recycling factor (RRF), EF-G and IF3. While RRF and EF-G are required for subunit dissociation, IF3 stimulates the dissociation of deacylated tRNA from the 30S P site and prevents subunit reassociation (Peske et al, 2005).

\section{The fidelity of protein biosynthesis}

Speed and accuracy of protein biosynthesis are fundamental parameters in the quality control of translation. Misincorporation of amino acids may reduce the amount of active proteins, produce proteins that are toxic for the cell, or increase the probability of misfolding. Estimations of error frequencies of translation range between $10^{-5}$ and $10^{-3}$, depending on the type of measurement, concentrations and nature of tRNAs that perform misreading, and the mRNA context (Drummond \& Wilke, 2009; Kramer \& Farabaugh, 2007; Parker, 1989). Different approaches were taken to measure these values. For instance, error frequencies of amino acid incorporation at a particular position of the protein were estimated based on different physicochemical properties of native and altered proteins or their fragments (Parker, 1989). Alternatively, error frequencies were obtained using reporter constructs expressing proteins which gain enzymatic activity upon misincorporation (Kramer \& Farabaugh, 2007; Sergiev et al, 2005). The overall measured error rate of protein synthesis reflects the accumulated mistakes from all steps involved in translation, of which tRNA aminoacylation and decoding arguably are the most error- 
prone. To prevent the incorporation of incorrect amino acids into proteins, the protein biosynthesis machinery employs numerous quality control mechanisms (Reynolds et al, 2010).

\subsection{Fidelity of aminoacylation and aa-tRNA binding to EF-Tu}

The first step at which the fidelity of protein biosynthesis is controlled is the aminoacylation of tRNA by aa-tRNA synthetases. Aminoacylation is a two-step mechanism. The first step is the activation of the amino acid with ATP to form an aminoacyl-adenylate (aa-AMP). During the second step the amino acid is transferred to the tRNA. Quality control mechanisms by aaRSs exist at both steps, the pre-transfer editing, i.e. the hydrolysis of mischarged aminoacyl-adenylates, and the post-transfer editing, i.e. the hydrolysis of mischarged aa-tRNAs in the editing site of the aaRS (Ling et al, 2009). Furthermore, several trans-editing factors exist which hydrolyze misacylated tRNA after its dissociation from the synthetase, e.g. the Haemophilus influenzae YbaK which is involved in Cys-tRNA ${ }^{\text {Pro }}$ hydrolysis (An \& Musier-Forsyth, 2005).

After aminoacylation of the tRNA by the aaRS, aa-tRNA is bound by EF-Tu.GTP, forming the ternary complex EF-Tu-GTP-aa-tRNA, which serves to protect the aa-tRNA from hydrolysis at physiological $\mathrm{pH}$ and makes it a substrate for protein synthesis on the ribosome. Structurally, EF-Tu is composed of an N-terminal G domain and two antiparallel $\beta$-barrel domains (Kjeldgaard \& Nyborg, 1992). The interaction of EF-Tu and aa-tRNA in the ternary complex involves interactions between the acceptor arm and the amino acid of the tRNA and all three domains of EF-Tu (Nissen et al, 1995). In the GTP form EF-Tu binds to all canonical aa-tRNAs with similar high affinities (Louie \& Jurnak, 1985; Louie et al, 1984; Ott et al, 1990), which initially suggested that EF-Tu is not able to discriminate the amino acids esterified to the acceptor ends of aa-tRNAs. This view might also have been supported by the seminal experiment showing that the translation of a poly-UG mRNA template (coding for cysteine) by a misacylated Ala-tRNA ${ }^{\mathrm{Cys}}$, produced by Raney Nickel reduction, resulted in incorporation of alanine into nascent peptides (Chapeville et al, 1962). A different case is observed in organisms which lack GlnRS and produce GlntRNA $^{\text {Gln }}$ via the transamidation pathway. This indirect aminoacylation pathway occurs in two steps. First, tRNA ${ }^{\text {Gln }}$ is misacylated by GluRS, then Glu-tRNA ${ }^{\text {Gln }}$ is converted to GlntRNA $^{\text {Gln }}$ by an amidotransferase (Ibba \& Söll, 2000). However, Glu is not misincorporated at Gln codons, because Glu-tRNA ${ }^{\text {Gln }}$ is not accepted by the translation machinery. It has been shown that EF-Tu binds Glu-tRNA ${ }^{\text {Gln }}$ only poorly (Stanzel et al, 1994), allowing the 
exclusion of this aa-tRNA from protein synthesis and an efficient competition for GlutRNA $^{\text {Gln }}$ by the amidotransferase. In experiments using different misacylated tRNAs it was shown that EF-Tu shows specificity for both the amino acid and the tRNA body (LaRiviere et al, 2001). Each amino acid side chain makes different interactions with the amino acid binding pocket of EF-Tu resulting in variable thermodynamic contributions to the binding interaction. It has been hypothesized that each tRNA sequence has been evolutionarily optimized to compensate for the contributions of the amino acid to result in a uniformly tight binding to EF-Tu (Dale \& Uhlenbeck, 2005a). This specificity might contribute to the fidelity of protein biosynthesis in a way that some misacylated aa-tRNAs might not form a stable ternary complex with EF-Tu, and are therefore excluded from ribosomal protein synthesis. On the other hand, misacylated aa-tRNAs could also form a very tight ternary complex with a higher stability than their canonical counterparts. These complexes are delivered to the ribosome but exhibit slower rates of peptide bond formation, presumably due to difficulties in rapid dissociation of the aa-tRNA from EF-Tu (Schrader et al, 2011).

\subsection{Fidelity mechanisms on the ribosome}

The fidelity of substrate selection on the ribosome is controlled by a multistep kinetic mechanism which has been described in detail using single molecule and bulk kinetic approaches (Marshall et al, 2008; Rodnina \& Wintermeyer, 2001; Wohlgemuth et al, 2011) (Figure 3). The first interaction of the ternary complex and the ribosome is established by contacts between EF-Tu and one of the multiple copies of the ribosomal protein L7/12 (Diaconu et al, 2005; Kothe et al, 2004). The interaction is highly dynamic, characterized by high association $\left(\mathrm{k}_{1}\right)$ and dissocation $\left(\mathrm{k}_{-1}\right)$ rates, and is independent of the codon presented in the ribosomal A site (Rodnina et al, 1996). The following step is codon reading $\left(\mathrm{k}_{\text {read }}, \mathrm{k}_{\text {-read }}\right)$ in which the aa-tRNA undergoes rapid conformational sampling, that allows the anticodon of the tRNA to interact with the codon presented in the decoding center of the ribosomal A site, while it still remains bound to EF-Tu (Blanchard et al, 2004; Geggier et al, 2010). The decoding center of the 30S subunit harbors the conserved residues A1492, A1493 and G530 of 16S rRNA. In the absence of a tRNA substrate A1492 and A1493 are not well ordered and stacked in the interior of an internal loop of helix 44 (Carter et al, 2000). Upon binding of a cognate tRNA substrate and formation of a stable codon-anticodon duplex $\left(\mathrm{k}_{2}, \mathrm{k}_{-2}\right)$, A1492 and A1493 flip out of the loop in helix 44 and monitor the Watson-Crick base pair geometry in the minor groove of the codonanticodon duplex through A-minor interactions (Figure 4). The first position of the codon- 
anticodon duplex is monitored by A1493, forming hydrogen bonds with the 2'OH of the bases of the codon and the anticodon. The second position is monitored by A1492, which forms hydrogen bonds with the 2'OH of the codon base and G530, which forms a hydrogen bond with the 2'OH of the tRNA base.

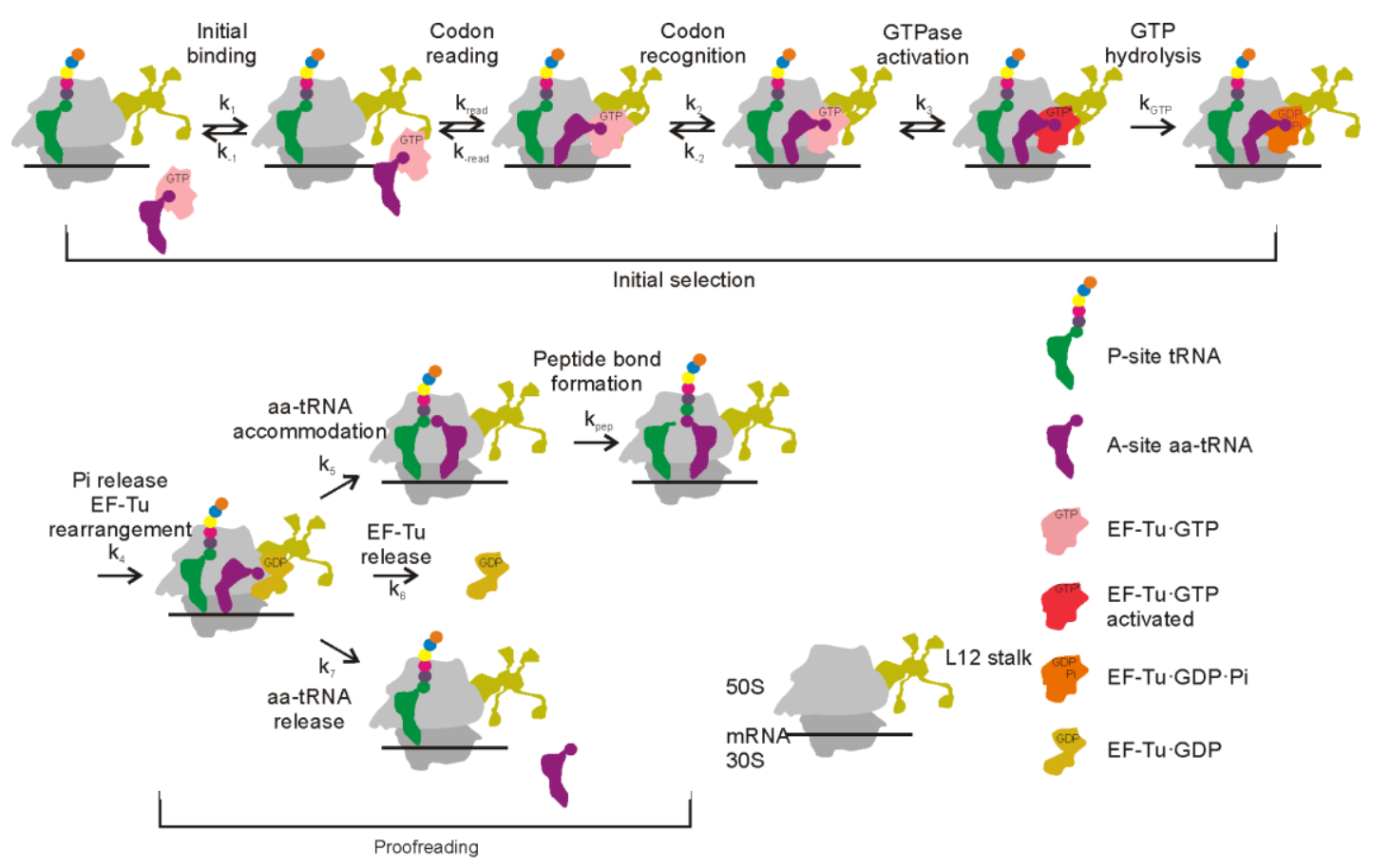

Figure 3 Schematic of EF-Tu-dependent aa-tRNA binding to the A site. Kinetically resolved steps are indicated by the rate constants $\mathrm{k}_{1}$ to $\mathrm{k}_{7}$ (forward reactions) and $\mathrm{k}_{-1}$ and $\mathrm{k}_{2}$ (backward reactions). The rate of codon reading (presumably a readily reversible step (Geggier et al, 2010)) could not be determined by rapid kinetics; the values available from single-molecule FRET experiments (Blanchard et al, 2004; Geggier et al, 2010) are not comparable with the values obtained in bulk experiments due to differences in experimental conditions. Rate constants of the two chemical steps (that are rate-limited by the respective preceding step) are designated $\mathrm{k}_{\mathrm{GTP}}$ and $\mathrm{k}_{\mathrm{pep}}$.

The third position is monitored less stringently as it accepts also non-canonical (wobble) base pairing. The position of the 2'OH of the codon base in the third position of the codonanticodon duplex is monitored by hydrogen bonds with G530, and indirectly by coordination of $\mathrm{a} \mathrm{Mg}^{2+}$ ion with C518 of 16S rRNA and Pro48 of ribosomal protein S12 (Ogle et al, 2001). These local structural changes in the 16S rRNA induce global conformational changes in the 30S subunit, causing a movement of its head and shoulder relative to each other (Ogle et al, 2002). This closure of the $30 \mathrm{~S}$ subunit occurs along with a series of conformational rearrangements in the aa-tRNA and EF-Tu, resulting in a distorted conformation of the aa-tRNA which allows the simultaneous interaction of aatRNA with the 30S decoding center and EF-Tu (Schmeing et al, 2009; Schuette et al, 2009; Stark et al, 2002; Valle et al, 2003; Villa et al, 2009). During the GTPase activation ( $\mathrm{k}_{3}$, $\mathrm{k}_{-3}$ ), which follows directly after the codon recognition step (Rodnina et al, 1995), all the 
residues necessary for catalyzing GTP hydrolysis in the active site of EF-Tu are oriented into a productive conformation. Histidine 84 plays an essential role for the catalysis, and mutation of this residue to alanine leads to a $10^{6}$-fold reduction in the rate of GTP hydrolysis (Daviter et al, 2003). The reaction involves the attack of a hydrolytic water molecule on the $\gamma$-phosphate of GTP. It has been suggested that a hydrophobic gate formed by residues Val20 and Ile60 prevents His84 from entering the active site, in the nonactivated form of the factor. Upon activation this gate is opened and His84 is allowed to enter and approach the catalytic water molecule (Krab \& Parmeggiani, 1999; Villa et al, 2009). Positioning of His 84 in the active site is furthermore facilitated by the interaction with A2662 of 23S rRNA (Voorhees et al, 2010).

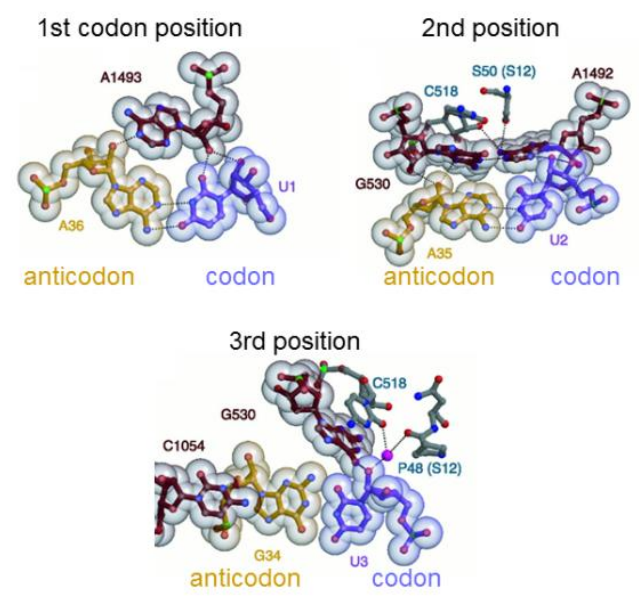

Figure 4 Interaction of the ribosome with the base pairs of the codon-anticodon duplex. In the first position of the codon A1493 of $16 \mathrm{~S}$ rRNA forms A-minor interactions with the minor groove of the A36-U1 base pair. In the second position G530 and A1492 both interact with the minor groove of the A35-U2 base pair. The third (wobble) position is monitored less stringently. Here, G530 interacts directly with U3 of the G34U3 base pair and C518 and Pro48 of ribosomal protein S12 interact indirectly with U3 by coordinating a $\mathrm{Mg}^{2+}$ ion (adapted from (Ogle et al, 2001)).

GTPase activation is followed by rapid GTP hydrolysis ( $\mathrm{k}_{\mathrm{GTP}}$ ) (Gromadski et al, 2006; Gromadski \& Rodnina, 2004a; Gromadski \& Rodnina, 2004b; Pape et al, 1999), the precise catalytic mechanism of which is still controversial. One possible mechanism involves His84 acting as a general base which abstracts a proton form the catalytic water molecule, which then attacks the $\gamma$-phosphate (Voorhees et al, 2010). Alternatively, the $\gamma$ phosphate itself might abstract a proton from the catalytic water (Liljas et al, 2011) and His84 might play a role in stabilizing the transition state (Adamczyk \& Warshel, 2011; Daviter et al, 2003).

Following GTP hydrolysis in EF-Tu phosphate is released from the factor, which then undergoes a major conformational rearrangement $\left(\mathrm{k}_{4}\right)$, leading to its dissociation $\left(\mathrm{k}_{6}\right)$ from aa-tRNA and the ribosome (Berchtold et al, 1993; Kothe \& Rodnina, 2006). This allows 
aa-tRNA to move into a fully accommodated A/A state $\left(\mathrm{k}_{5}\right)$ and take part in peptide bond formation $\left(\mathrm{k}_{\mathrm{pep}}\right)$, or be rejected $\left(\mathrm{k}_{7}\right)$ during the proofreading step. Finally, EF-Tu.GDP is converted back to its GTP form with the help of EF-Ts which is a guanine-nucleotide exchange factor (for review see (Vetter \& Wittinghofer, 2001)) facilitating the exchange with GTP (Gromadski et al, 2002) and allowing it to bind the next aa-tRNA.

In a number of studies the elemental rate constants which govern the selection of cognate and near-cognate ternary complex on the ribosome have been determined (Gromadski et al, 2006; Gromadski \& Rodnina, 2004a; Pape et al, 1999). Four elemental reactions have different rates for cognate and near-cognate aa-tRNAs, whereas others are very similar (Table 1).

Table1 Rate constants of elemental steps of decoding the cognate UUU and near-cognate CUC codons by Phe-tRNA $^{\text {Phe }}$ (HiFi buffer, $20^{\circ} \mathrm{C}$ ) (Gromadski et al, 2006; Gromadski \& Rodnina, 2004a). The $\mathrm{k}_{6}$ value (Figure 3) was not measured at HiFi conditions ( $3.5 \mathrm{mM} \mathrm{Mg}^{2+}$, polyamines); at $10 \mathrm{mM} \mathrm{Mg}^{2+}, \mathrm{k}_{6}=3 \mathrm{~s}^{-1}$.

\begin{tabular}{lrr}
\hline Rate constant & UUU & CUC \\
\hline $\mathrm{k}_{1}, \mu \mathrm{M}^{-1} \mathrm{~s}^{-1}$ & 140 & 140 \\
$\mathrm{k}_{-1}, \mathrm{~s}^{-1}$ & 85 & 85 \\
$\mathrm{k}_{2}, \mathrm{~s}^{-1}$ & 190 & 190 \\
$\mathrm{k}_{-2}, \mathrm{~s}^{-1}$ & 0.2 & 80 \\
$\mathrm{k}_{3}, \mathrm{~s}^{-1}$ & 260 & 0.4 \\
$\mathrm{k}_{4}, \mathrm{~s}^{-1}$ & $\geq 10^{\mathrm{a}}$ & $\mathrm{n} . \mathrm{d}$. \\
$\mathrm{k}_{5}, \mathrm{~s}^{-1}$ & 20 & $0.26^{\mathrm{b}}$ \\
$\mathrm{k}_{7}, \mathrm{~s}^{-1}$ & $<0.3$ & $7^{\mathrm{b}}$ \\
\hline
\end{tabular}

${ }^{\mathrm{a}}$ The value was measured at $1 \mu \mathrm{M}$ substrate concentration (Mittelstaet et al, 2011).

b The values cannot be determined at $20^{\circ} \mathrm{C}$ in $\mathrm{HiFi}$ buffer, because the preceding GTP hydrolysis step is strongly rate-limiting (Gromadski et al, 2006). Values given are for $\mathrm{HiFi}$ at $37^{\circ} \mathrm{C}$ (Wohlgemuth et al, 2010). Similar values $\left(\mathrm{k}_{5}=0.1 \mathrm{~s}^{-1}, \mathrm{k}_{7}=6 \mathrm{~s}^{-1}\right)$ were obtained at $10 \mathrm{mM} \mathrm{MgCl}_{2}$ without polyamines at $20^{\circ} \mathrm{C}$ (Pape et al, 1999).

The ribosome controls the differences in the stabilities of the codon-anticodon complexes $\left(\mathrm{k}_{-2}, \mathrm{k}_{7}\right)$ and specifically increases the rate constants of GTPase activation $\left(\mathrm{k}_{3}\right)$ and accommodation $\left(\mathrm{k}_{5}\right)$ for correct substrates, implicating both an increased stability of tRNA binding and induced fit as sources of selectivity (Rodnina \& Wintermeyer, 2001). Kinetic partitioning between GTPase activation and ternary complex dissociation strongly favors the acceptance of cognate and rejection of near-cognate ternary complexes. Likewise, cognate aa-tRNA is preferentially accommodated during proofreading while near-cognate tRNA is largely rejected. Furthermore, it has been discovered recently that the fidelity of protein biosynthesis is also controlled at the level of peptidyl-tRNA (Zaher \& Green, 2009b). 


\section{Codon recognition}

Base pairs in the codon-anticodon duplex of near-cognate substrates deviate from Watson-Crick geometry and cannot form hydrogen bonds with the hydrogen-bonding partners at the codon-anticodon minor groove (Ogle et al, 2001; Ogle et al, 2002) (Figure 4). This by itself leads to a relatively unstable codon-anticodon interaction on the $30 \mathrm{~S}$ subunit relative to cognate substrates. Moreover, near-cognate tRNAs fail to induce the structural rearrangements in the $30 \mathrm{~S}$ subunit leading to its closure and stabilization of the tRNA on the ribosome (Ogle et al, 2002). Further support of the importance of conformational changes in the $30 \mathrm{~S}$ subunit for the induced fit mechanism as a source of selectivity, comes from studies using antibiotics. Streptomycin has been shown to induce misreading (Davies et al, 1964) by decreasing the rate of GTP hydrolysis for cognate substrates and accelerating the rate of GTP hydrolysis for near-cognate substrates (Gromadski \& Rodnina, 2004b). This effect might be explained by a restriction of the conformational flexibility of the $30 \mathrm{~S}$ subunit by streptomycin which interferes with subunit closure but sustains a moderate level of GTPase activation for both cognate and nearcognate substrates (Carter et al, 2000; Gromadski \& Rodnina, 2004b). Paromomycin, which also induces misreading, has been shown to induce structural rearrangements of the 30S subunit typical for a cognate codon-anticodon interaction also in the presence of nearcognate substrates (Ogle et al, 2001; Ogle et al, 2002), resulting in acceleration of the rate of GTP hydrolysis for near-cognate substrates, while the rate of GTP hydrolysis of cognate substrates remains unaffected (Gromadski \& Rodnina, 2004b; Pape et al, 2000). Recently, the importance of subunit closure in the selection of cognate substrates was challenged by a study in which it was shown that the structural changes in the 30S subunit can also be induced by near-cognate substrates (Demeshkina et al, 2012), albeit at conditions where the ribosome was not able to proofread the incorrect substrates.

\section{GTPase activation}

During GTPase activation, which is the rate limiting step for GTP hydrolysis, the conformational signal of cognate codon-anticodon interaction on the $30 \mathrm{~S}$ subunit has to be transmitted to the active site of EF-Tu where GTP hydrolysis takes place. The decoding center of the 30S subunit and the GTP binding pocket of EF-Tu on the 50S subunit are located almost $80 \AA$ apart from each other. How exactly cognate codon-anticodon interaction accelerates GTP hydrolysis in EF-Tu is not known. It has been shown that an intact but distorted aa-tRNA is required for the efficient GTPase activation in EF-Tu, 
suggesting that the tRNA plays an active role in the transmission of the signal (Cochella $\&$ Green, 2005; Piepenburg et al, 2000). The distorted conformation of cognate aa-tRNA in the $\mathrm{A} / \mathrm{T}$ state of decoding has been detected using transient kinetic approaches based on fluorescence changes of a proflavin label introduced at the elbow region of aa-tRNA (Rodnina et al, 1994). Furthermore, it has been observed in structural studies of stalled ribosomal complexes in presence of kirromycin (Schmeing et al, 2009; Schuette et al, 2009; Stark et al, 2002; Valle et al, 2003; Villa et al, 2009), an antibiotic that stabilizes the ternary complex on the ribosome without affecting any of the steps prior to GTP hydrolysis and phosphate release (Kothe \& Rodnina, 2006), or in the presence of GDPCP (Voorhees et al, 2010), a non-hydrolyzable GTP analogue. The distortion of the aa-tRNA involves changes in the position of the $\mathrm{D}$ stem relative to the $\mathrm{T}$ stem along with a partial unfolding of the D loop and a reduction of the helical twist in the anticodon stem (Schmeing et al, 2009; Schuette et al, 2009) (Figure 5). Moreover, the position of the 3' end of aa-tRNA (residues 72-75), which interacts with EF-Tu, is displaced in comparison to the free ternary complex (Nissen et al, 1995).
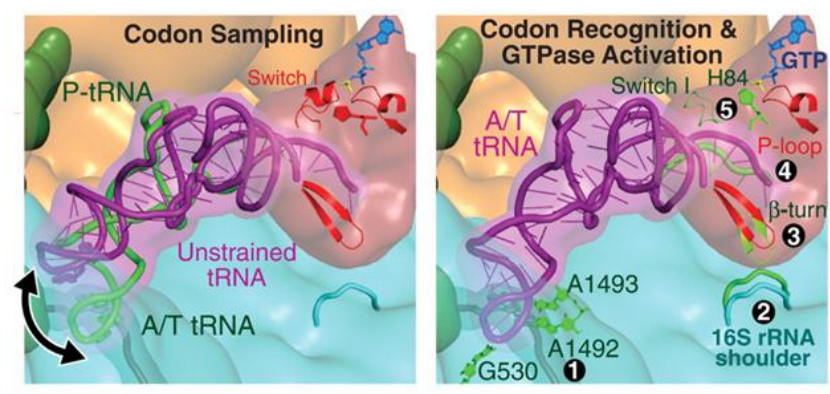

Figure 5 Distorted conformation of aa-tRNA on the ribosome. (Left panel) Distortion induced during codon reading. (Right panel) Structural rearrangements induced by cognate codon-anticodon interaction (for details see text). The formation of the codon-anticodon duplex is monitored by residues A1492, A1493, and G530 of $16 \mathrm{~S}$ rRNA (1). 30S domain closure is triggered (2), which brings the $30 \mathrm{~S}$ shoulder into contact with EF-Tu and shifts the position of the $\beta$ loop (res 230-237) of domain 2 (3). The conformation of the acceptor end of aa-tRNA changes (4), resulting in the disruption of contacts with the switch I region of EF-Tu, which then becomes disordered (5), and positions His84 into the active site for GTP hydrolysis (adapted from (Schmeing et al, 2009)).

The distorted conformation of the aa-tRNA is stabilized by contacts with the shoulder domain of 16S rRNA, ribosomal protein S12 (Schmeing et al, 2009; Stark et al, 2002) and the L11 region of 23S rRNA (Schmeing et al, 2009). Functionally, the distortion changes the relative orientation of EF-Tu and tRNA and might induce structural rearrangements in the factor that influence the precise positioning of EF-Tu at the sarcin-ricin loop of $23 \mathrm{~S}$ rRNA, facilitating the interaction of His84 with A2662 (Voorhees et al, 2010). 
An important role of the tRNA distortion in the decoding mechanism is supported by studies of tRNAs with mutations distant from the anticodon loop. The most prominent example is the Hirsh suppressor tRNA ${ }^{\text {Trp }}$ which has a G24A mutation in the D stem and exhibits an increased readthrough of UGA stop codons (Hirsh, 1971; Hirsh \& Gold, 1971). Kinetic studies have shown that in the presence of the G24A mutation, GTP hydrolysis and peptide bond formation are accelerated on the UGA codon in comparison to the wt tRNA $^{\text {Trp }}$ (Cochella \& Green, 2005). Guanine at position 24 is normally paired to U11 in the $\mathrm{D}$ stem, and the mutation to adenine allows the formation of an additional hydrogen bond of A24 to G44 which was suggested to stabilize the distorted conformation of the tRNA on the ribosome (Schmeing et al, 2011). A UV-induced crosslink between residues 8 and 13, which lie inside the loop of the tRNA elbow close to the G24A, is able to suppress the misreading phenotype of the G24A mutation (Vacher \& Buckingham, 1979), probably by disfavouring the A24 to G44 interaction. Another example of a mutation distant from the anticodon, but causing suppression of the UGA stop codon by tRNA ${ }^{\text {Trp }}$ is the A9C mutation (Smith \& Yarus, 1989a; Smith \& Yarus, 1989b). The A9 base is normally involved in formation of a base triple with residues U12 and A23 in the D stem. Disruption of this interaction by the $\mathrm{A} 9 \mathrm{C}$ mutation, results in the destabilization of this base triple, which has been suggested to render the tRNA more flexible (Schmeing et al, 2011) and allow it to sample the distorted conformation more easily.

Given these examples, it seems that the distortion of the aa-tRNA might be a precisely tuned mechanism for the activation of the GTPase in EF-Tu on the ribosome, and specific for cognate substrates. However, recent dynamic fluorescence quenching experiments suggested that near-cognate codon-anticodon interaction, which does not induce efficient GTPase activation, leads to a similar distortion of the elbow region of aa-tRNA as observed on the cognate codon (Mittelstaet et al, 2011(Chapter I)). The opening of the elbow region of aa-tRNA was furthermore supported by a structural study of a nearcognate ternary complex stalled on the ribosome by kirromycin (Agirrezabala et al, 2011). In light of these experiments it seems that the distortion of aa-tRNA in the codon recognition complex might be a necessary but non-exclusive trigger for GTPase activation in EF-Tu. Rather, GTPase activation of EF-Tu on the ribosome seems to involve a finetuned global response mechanism, which involves conformational changes of both the $30 \mathrm{~S}$ subunit and the aa-tRNA, resulting in a precise docking of EF-Tu on the sarcin-ricin loop and allowing an efficient interaction of A2662 and His84. In this framework, tRNA mutants that facilitate activation of GTP hydrolysis on a near-cognate codon appear to 
have found their own unique conformational solution to position EF-Tu His84 close to A2662 of the sarcin-ricin loop (Voorhees et al, 2010). Furthermore, other contacts in the codon recognition complex might affect the stringency of decoding, such as helix 8 and 14 of $16 \mathrm{~S}$ rRNA, which negatively regulate GTP hydrolysis (McClory et al, 2010), or the interactions between helix 5 and domain 2 of EF-Tu (Vorstenbosch et al, 1996). Finally, the ribosome may play an active role in monitoring the correct codon-anticodon interaction using a network formed by helices 18 and 44 of 16S rRNA, helices 38, 69 and 89 of $23 \mathrm{~S}$ rRNA, and proteins S13, S19, L16, L25, L27 and L31 from both ribosomal subunits, as suggested by the crystal structure of a ribosome-tRNA complex in the proofreading stage (Jenner et al, 2010a).

\section{Accommodation and proofreading}

Accommodation involves a large-scale displacement of the 3' acceptor end of the aatRNA from EF-Tu to the A site of the 50S subunit by about $90 \AA$ along with a rotation of the tRNA around the anticodon stem loop axis, and can be considered as the rate-limiting step for peptide bond formation (Pape et al, 1999; Wohlgemuth et al, 2010). The trajectory of aa-tRNA movement during accommodation was studied in molecular dynamics simulations (Sanbonmatsu et al, 2005; Whitford et al, 2010) and described as a stepwise movement, in which the tRNA interacts with various conserved rRNA bases forming an accommodation corridor. It has been suggested that accommodation involves reversible excursions of the aa-tRNA along multiple pathways (Whitford et al, 2010), and that the precise positioning of the acceptor arm in the accommodation corridor, which is facilitated by a correct codon-anticodon interaction, is important for high accommodation rates. On the other hand, incorrect codon-anticodon interaction might cause a misalignment of the acceptor arm resulting in slower accommodation rates for near-cognate substrates (Sanbonmatsu, 2006). The final accommodation event furthermore involves an interaction of the acceptor end of aa-tRNA with the universally conserved bases U2492, C2556, and C2573 of 23S rRNA (Kim \& Green, 1999). These residues were proposed to act as a gate stalling the acceptor stem of aa-tRNA before it enters the peptidyl transferase center (Sanbonmatsu et al, 2005). Indeed mutations of bases U2492 and U2555 at the accommodation gate have been reported to decrease the fidelity of translation (O'Connor \& Dahlberg, 1993; O'Connor \& Dahlberg, 1995). However, mutations of C2573 and the neighbouring A2572 did not affect aa-tRNA accommodation, peptide bond formation, or the fidelity of protein synthesis (Burakovsky et al, 2010), suggesting that the misalignment 
of near-cognate tRNAs during accommodation might be monitored by a more sophisticated network of interactions with rRNA and proteins from the $50 \mathrm{~S}$ and $30 \mathrm{~S}$ subunits (Jenner et al, 2010a).

\section{Retrospective editing}

The fidelity of protein synthesis is also controlled at the level of the peptidyl-tRNA. If an amino acid from a non-cognate tRNA is incorporated into the nascent polypeptide, a general loss of specificity of the A site is observed, which leads to a reduced fidelity of subsequent incorporations and a stimulation of release factor-dependent termination of polypeptide synthesis (Zaher \& Green, 2009b). The rate of retrospective editing is further enhanced by RF3, which was recently shown to be important for controlling the fidelity of protein biosynthesis in vivo (Zaher \& Green, 2011). The fact that the GTPase activation and accommodation of near-cognate aa-tRNAs is accelerated, leading to an incorporation of near-cognate aa-tRNA with similar efficiency as cognate tRNAs, has led to the suggestion that the structural integrity of the codon-anticodon duplex in the $\mathrm{P}$ site can directly influence the specificity of the A site (Zaher \& Green, 2010).

\subsection{Bypassing of translational fidelity mechanisms to incorporate non-canonical amino acids into proteins}

The protein biosynthetic machinery has been evolutionarily optimized for the incorporation of 20 standard amino acids into proteins. Furthermore, two additional proteinogenic amino acids pyrrolysine and selenocysteine have been recognized (Driscoll \& Copeland, 2003; Krzycki, 2005). The extension of the genetic code by incorporation of non-canonical amino acids, which represent a chemically more diverse repertoire of protein building blocks, is an emerging field of synthetic biology and provides a means to design proteins with novel functions (for reviews see (Davis \& Chin, 2012; Johnson et al, 2010)). One of the first examples of protein biosynthesis using artificial amino acids was the substitution of methionine by selenomethionine, which later on proved to be a valuable tool for structure elucidation in X-ray crystallography (Cowie \& Cohen, 1957; Hendrickson et al, 1990). To date, the set of non-canonical amino acids which have been incorporated into proteins was greatly expanded by photocrosslinkers to study protein interactions (Chin et al, 2002), photocaged amino acids to activate functions in response to light (Lemke et al, 2007), fluorescent amino acids (Kajihara et al, 2006; Lepthien et al, 2008; Woolhead et al, 2004), post-translational modifications such as phosphoserine (Park 
et al, 2011), spectroscopic probes (Ye et al, 2010), and many others. The incorporation of a non-canonical amino acid can be residue-specific, i.e. all residues of a certain type are substituted by a non-canonical amino acid over an organism's whole proteome. These approaches often utilize auxotrophic host strains, which are defective in the biosynthetic pathway for the synthesis of a particular amino acid. Alternatively, non-canonical amino acid incorporation can be site-specific, meaning that only one amino acid which is encoded by a unique codon, e.g. a UAG stop codon, is substituted. The latter approach is based on the use of orthogonal tRNA/aaRS pairs, which specifically charge the non-canonical amino acid onto a unique tRNA (Wang et al, 2001; Xie \& Schultz, 2005).

Important limitations to the incorporation of non-canonical amino acids, independent of the strategy used, are defined by the same mechanisms that control the fidelity of translation and favor the incorporation of canonical amino acids into proteins. The first and probably most stringent stage at which the identity of the amino acid is monitored is the binding and activation of the amino acid by the aminoacyl-tRNA synthetase. Each of those enzymes harbours a highly selective amino acid binding pocket which leaves little room for structural heterogeneity and can effectively discriminate against non-cognate amino acids (Figure 6). Furthermore, some aaRS employ post-transfer editing mechanisms to hydrolyze incorrectly aminoacylated tRNAs (Ling et al, 2009). In order to be efficiently incorporated into proteins, non-canonical amino acids have to pass these selection stages. To improve the incorporation of non-canonical amino acids different approaches have been used ranging from increasing the cellular activity of a particular aaRS simply by its overexpression (Kiick et al, 2000; Tang \& Tirrell, 2001), to changing the substrate specificity of the aaRS by rational design, which is facilitated by the availability of the crystal structures for most aaRS (Kast \& Hennecke, 1991; Tang et al, 2009). Moreover, directed evolution of aaRSs has been used to improve the acceptance for certain noncanonical substrates (Neumann et al, 2008; Tanrikulu et al, 2009; Xie \& Schultz, 2005).

Once the tRNA has been charged with a non-canonical amino acid it has to bind to EF-Tu. As EF-Tu binds to all elongator aa-tRNAs, the amino acid binding pocket is spacious and allows more flexibility in the binding of different amino acids, compared to most aaRS (Figure 6). However, given that the affinity of aa-tRNA to EF-Tu is characterized by fine-balanced contributions of the tRNA sequence and the amino acid side chain (Dale \& Uhlenbeck, 2005a), it seems likely that non-canonical amino acids can influence this interaction. Examples of this are the low affinity of tRNAs aminoacylated with pyrenylalanine, phosphoserine, and a fluorescent derivative of lysine to EF-Tu, 
preventing the formation of a stable ternary complex (Doi et al, 2007; Park et al, 2011; Chapter II).
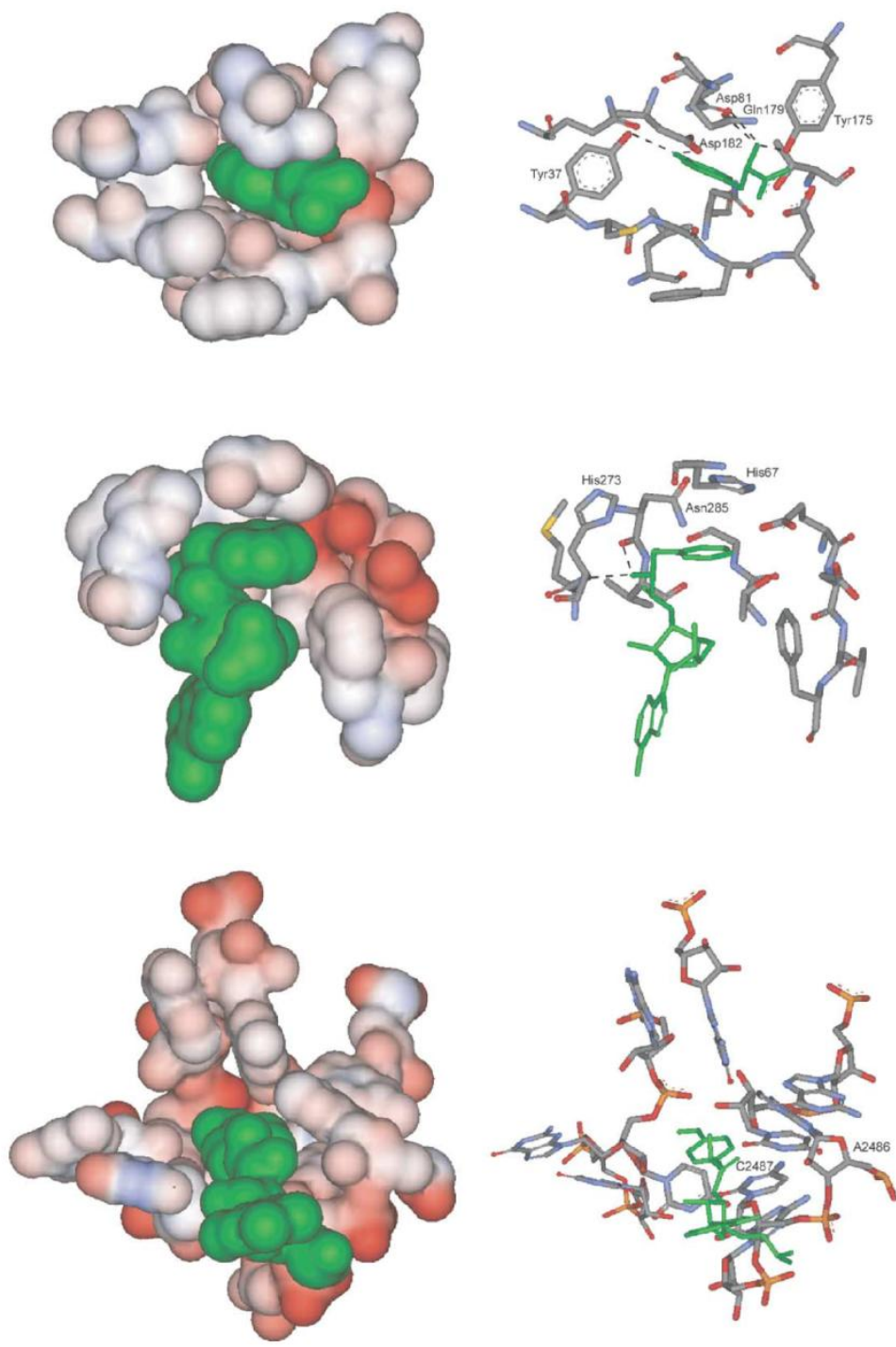

Figure 6 Amino acid-binding pockets of an aaRS, EF-Tu, and the ribosome viewed from the solvent accessible site. (Top) Tyrosine binding pocket of E. coli TyrRS ((Kobayashi et al, 2005), PDB code: 1X8X). The surface representation shows a precise fit of tyrosine (green) in the substrate binding pocket of the enzyme (colours by electrostatic potential: red, negative, blue, positive). In the stick representation (right) putative hydrogen bonds between the protein and the p-hydroxyl group and the $\alpha$-amino group of tyrosine, which might aid in substrate binding and discrimination, are highlighted. (Middle) Amino acid-binding pocket of Thermus aquaticus EF-Tu ((Nissen et al, 1995), PDB code: 1TTT). Phenylalanine and the 3 'terminal adenosine are shown in green. The amino acid-binding pocket of EF-Tu is rather large, as it has to accommodate all 20 different amino acids. The $\alpha$-amino group of phenylalanine forms hydrogen bonds with residues Asn285 and His273 of EF-Tu. These interactions can be formed by all amino acids except proline. (Bottom) A site of the 50S subunit of Haloarcula marismortui ribosomes ((Schmeing et al, 2002), PDB code: $1 \mathrm{KQS}$ ) bound to puromycin (green), which is an analogue to the 3'terminal adenosine and esterified amino acid of aa-tRNA. The asymmetric hydrophobic cleft formed by $23 \mathrm{~S}$ rRNA residues can easily accommodate the aromatic p-methoxyphenyl side chain of puromycin but might exhibit some substrate specificity (adapted from (Dale \& Uhlenbeck, 2005a)).

Similar to the approaches used to increase aminoacylation efficiency using non-canonical amino acids, site-specific mutagenesis and directed evolution of residues in the amino acid binding pocket of EF-Tu were used to improve the affinity to tRNAs aminoacylated to 
non-canonical amino acids (Chapman et al, 2012; Doi et al, 2007; Park et al, 2011). Alternatively, EF-Tu concentration can be optimized to promote ternary complex formation and efficient incorporation of fluorescent non-canonical amino acids into peptides using in vitro translation systems (Chapter II, Chapter IV). Furthermore, tRNA nucleotides at the interaction surface with EF-Tu have been subjected to directed evolution, resulting in tRNA species exhibiting improved incorporation efficiencies for different non-canonical amino acids (Guo et al, 2009).

In order to pass selection by the ribosome, tRNAs aminoacylated to non-canonical amino acids must be efficiently released from EF-Tu and accommodate in the $50 \mathrm{~S}$ ribosomal A site. Experiments using derivatives of the low molecular weight aa-tRNA analogue puromycin, indicate that the A site on the $50 \mathrm{~S}$ subunit shows some specificity for the amino acid side chain (Bhuta et al, 1981; Starck et al, 2003). This is supported by crystal structures of the 50S subunit bound to puromycin, which show that the amino acid side chain is bound to a hydrophobic cleft which might exhibit some substrate specificity (Nissen et al, 2000; Schmeing et al, 2002) (Figure 6). The binding of aa-tRNA to the A site on the 70S ribosome is also dependent on the nature of the amino acid side chain, however only in cases of a weak codon-anticodon interaction (Dale et al, 2009; Dale \& Uhlenbeck, 2005b). In fact, native tRNAs misacylated to different natural amino acids are efficiently selected as substrates by the ribosome (Effraim et al, 2009), indicating that the specific contribution of the amino acid side chain to A-site binding is relatively small compared to the contribution of the tRNA body. This is consistent with the observation that peptide bond formation with a fluorescent derivate of lysine esterified to a native tRNA ${ }^{\text {Lys }}$ can occur at rates comparable to these of natural aa-tRNAs into peptides (Chapter II, Chapter IV). Furthermore, by synthetic evolution of $16 \mathrm{~S}$ rRNA orthogonal ribosomes have been created which exhibit an improved decoding of UAG stop codons and quadruplet codons by nonsense and frameshift suppressor tRNAs and can be used to direct non-canonical amino acid incorporation to unique codons on orthogonal mRNAs (Neumann et al, 2010; Wang et al, 2007). 


\section{Non-uniform rates of protein synthesis}

Translation of cellular mRNAs proceeds through repetitive cycles of the elongation phase (Figure 2). The rate of protein elongation in bacteria is between 4 and 22 amino acids per second at $37^{\circ} \mathrm{C}$ (Liang et al, 2000; Mitarai et al, 2008; Proshkin et al, 2010; Sorensen \& Pedersen, 1991); thus a protein of an average length of 330 amino acids (Netzer \& Hartl, 1997) is completed in about 10-80 s. The intrinsic rates governing the selection of different aa-tRNAs on their respective cognate codons are very similar (Ledoux \& Uhlenbeck, 2008), with the exception of Pro-tRNA ${ }^{\text {Pro }}$, which has been shown to exhibit slower rates peptide bond formation (Pavlov et al, 2009). Furthermore, the rate of GTP hydrolysis by EF-Tu can vary 2.5-fold on a cognate codon depending on the presence of a Watson-Crick or a wobble base pair in the third position of the codonanticodon duplex (Gromadski et al, 2006; Thomas et al, 1988). Despite the intrinsic uniformity of rates governing the basic steps of substrate selection on the ribosome, translation elongation has been suggested to be a non-uniform process characterized by discontinuous elongation rates (Varenne et al, 1984).

The reasons for the discontinuity of elongation may be manifold. First, the arrival of a particular aa-tRNA at the A site is limited by diffusion of the ternary complex through the cytoplasm. Due to considerable variations in cellular concentrations of individual tRNA isoacceptors (Dong et al, 1996; Ikemura, 1985), diffusion processes can result in different arrival times of particular ternary complexes at the A site (Fluitt et al, 2007). Given the redundancy of the genetic code, synonymous triplets coding for the same amino acid can be decoded with different rates depending on the concentration of the corresponding aatRNA. In this way codon usage can determine local translation rates (Sorensen et al, 1989). Particularly clusters of rare codons have been suggested to cause transient pausing of translation elongation (Clarke \& Clark, 2008; Komar, 2009; Zhang et al, 2009). Aa-tRNA concentrations in the cell are further influenced by the availability of amino acids and may change under conditions of amino acid starvation (Dittmar et al, 2005). Stable mRNA secondary structures which need to be resolved by the intrinsic helicase activity of the ribosome can be yet another source of non-uniform translation rates ( $\mathrm{Qu}$ et al, 2011; Takyar et al, 2005; Wen et al, 2008). Furthermore, electrostatic interaction of positively charged amino acids in the nascent polypeptide chain with the negatively charged ribosomal tunnel can slow down elongation kinetics (Lu \& Deutsch, 2008). Recently, it was suggested that the interaction of the ribosomal anti-Shine-Dalgarno sequence of $16 \mathrm{~S}$ rRNA with internal Shine-Dalgarno-like sequences of mRNAs is a major cause of 
translational pausing ( $\mathrm{Li}$ et al, 2012). While all of these factors may contribute to the nonuniformity of translation in minimal model systems of protein biosynthesis in vitro (Chapter III), additional factors certainly play a role in the cell. One important factor is that single mRNA molecules can be translated by multiple ribosomes which can result in the formation of polysomes in which ribosomes assume tightly packed spatial organization forms (Brandt et al, 2009), but can also lead to collisions between individual ribosomes which thereby mutually influence their translation speed (Mitarai et al, 2008). Moreover, translation rates might also be affected by cooperation between the RNA polymerase machinery and translating ribosomes (Burmann et al, 2010; Proshkin et al, 2010). Variation of the rate of protein biosynthesis is functionally important for many cellular processes such as metabolic regulation of gene expression (Yanofsky, 2004). Moreover, it was suggested that transient pausing events during protein chain elongation can have an important role in guiding nascent chain protein folding on the ribosome (Komar, 2009; Purvis et al, 1987). Finally, it might be important to note that virtually all processes that govern translation elongation, probably except the chemical steps of GTP hydrolysis and peptide bond formation, are governed by thermal fluctuations and diffusion processes. In particular, the random diffusion of ternary complexes through the medium may contribute to a stochastic behavior of ribosomes during translation elongation (Garai et al, 2009) (Chapter III) and might have impact on all co-translational processes that depend on variations of the elongation rate.

\section{Co-translational protein folding}

How proteins fold into their native three-dimensional structures is one of the most exciting questions in modern biology. Since Christian Anfinsen's postulate that the amino acid sequence of a protein contains all the information necessary to specify its native three dimensional structure (Anfinsen, 1973) many of the principles underlying this complex process have been investigated in studies of protein unfolding and renaturation in vitro. Yet, protein folding cannot be completely understood without the biological context of protein chain elongation on the ribosome.

Protein folding, at least for proteins of more than 100 aa, is characterized by the formation of intermediate states along the folding pathway (Brockwell \& Radford, 2007) before the native state with its global free energy minimum is reached. Studies of protein chain renaturation in vitro have shown that formation of some intermediates is commonly observed on a millisecond time scale (Roder \& Colon, 1997). Given the rate of protein 
synthesis on the ribosome, protein folding in vivo - at least for the formation of secondary and simple tertiary structures - is rate-limited by polypeptide synthesis on the ribosome (Cabrita et al, 2010; Fedorov \& Baldwin, 1997). The nature of the synthesis and the rate of protein folding events therefore define protein folding as a vectorial process, which can involve sequential structuring of the nascent polypeptide on the ribosome. Co-translational folding is thought to allow nascent chains to retain an overall lower conformational energy and to avoid kinetic traps (local energy minima) that might be entered during refolding of a completely unstructured polypeptide in solution (Figure 7).

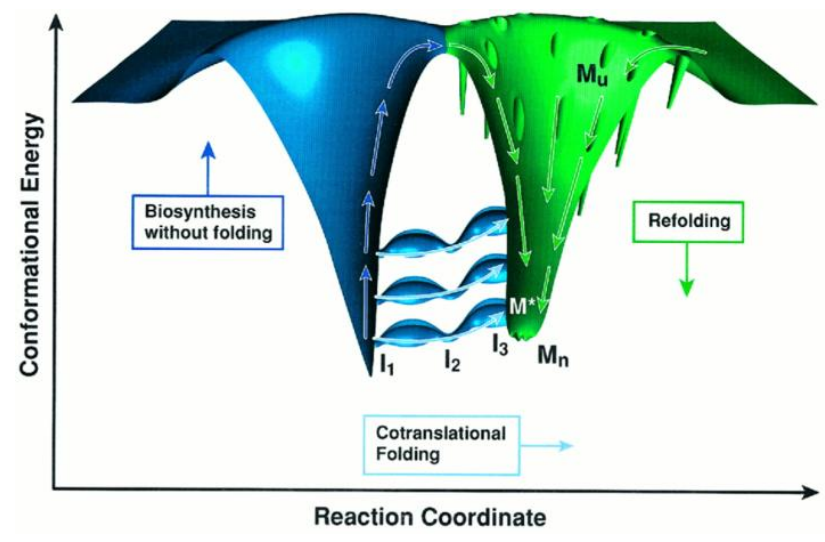

Figure 7 The concept of protein biosynthesis in the absence of folding compared to co-translational protein folding. The overall reaction coordinate of protein biosynthesis proceeds from left to right. Polypeptide synthesis in the absence of folding is represented by the blue surface. The circumference of the funnel shows the conformational space available to the nascent chain. The green surface represents the formation of noncovalent interactions in the polypeptide which are associated with protein folding. Co-translational protein folding proceeds through several intermediates $\left(I_{1}, I_{2}, I_{3}\right)$ and is represented by tunnels, connecting the left and the right funnel. $\mathrm{Mu}$, is the unfolded protein, $\mathbf{M}^{*}$, the full length nascent polypeptide after cotranslational folding, and $\mathrm{Mn}$ is the native state of the protein (adapted from (Fedorov \& Baldwin, 1997)).

In this way co-translational protein folding can reduce the complexity of folding pathways, and in many cases should be faster and more efficient than refolding. Indeed, this notion is supported by experiments comparing protein folding rates on the ribosome with refolding rates of denatured proteins in solution (Fedorov \& Baldwin, 1999; Kolb et al, 1994). Another feature of co-translational protein folding is defined by the structure of the ribosome. The ribosomal exit tunnel has a length of $80-100 \AA$ and a diameter of $10-20$ $\AA$ (Ban et al, 2000) and can accommodate polypeptides of about 30 aa assuming an extended conformation. While some structures are able to fold in the confined space of the ribosomal tunnel e.g. $\alpha$-helices (Lu \& Deutsch, 2005a; Lu \& Deutsch, 2005b; Woolhead et al, 2004), many other folding events, e.g. formation of $\beta$-sheets are excluded. Due to the large size of ribosomes, partially folded proteins are prevented from rapid diffusion through the cytoplasm, which minimizes their potential for aggregation (Fedorov \& 
Baldwin, 1997). Furthermore, ribosomes can associate with chaperones like trigger factor (Ferbitz et al, 2004), which maintain nascent polypeptide chains in a non-aggregated state and inhibit premature misfolding (Hartl et al, 2011).

Co-translational protein folding has been demonstrated in a number of studies using different experimental approaches (for review see (Fedorov \& Baldwin, 1997; Komar, 2009). These observations range from the detection of early tertiary structures in the nascent protein chain e.g. by monitoring the formation of disulphide bonds (Bergman \& Kuehl, 1979; Peters \& Davidson, 1982) to the detection of folded domains by NMR spectroscopy (Cabrita et al, 2009; Hsu et al, 2007) or by fluorescence depolarization decay measurements (Ellis et al, 2008; Weinreis et al, 2010). Furthermore, co-translational incorporation of fluorescently modified amino acids was used to detect protein folding inside and outside of the ribosomal tunnel by fluorescence resonance energy transfer (FRET) (Khushoo et al, 2011; Woolhead et al, 2004). Probably the most elegant way to monitor co-translational protein folding is the detection of ligand-binding or enzymatic activity which is specific for the folded protein in the ribosome-bound nascent chain. Examples for such experiments are the binding of heme to nascent chains of $\alpha$-globin or the binding of ATP to the nucleotide binding domain 1 of the cystic fibrosis transmembrane conductance regulator (Khushoo et al, 2011; Komar et al, 1997). Another example is the Semliki Forest virus capsid protein, which contains a chymotrypsin-like protease domain that folds into its active conformation while it is still attached to the ribosome, and thereby allows its own cleavage from a larger polyprotein precursor (Nicola et al, 1999). With firefly luciferase no enzymatic activity is detected while the nascent chain is attached to the ribosome, however upon its release the activity increases within a few seconds, while obtaining renatured protein after in vitro denaturation takes minutes, suggesting a sequential co-translational folding pathway (Frydman et al, 1999; Kolb et al, 1994). Indeed if the structural constraints of the ribosomal tunnel in these experiments are bypassed by extension of the nascent protein chain at the $\mathrm{C}$-terminal end, enzymatic activity of firefly luciferase can be detected in the nascent chain which is still bound to the ribosome (Makeyev et al, 1996). Similar to the latter example, the fluorescence of GFP derivatives has been detected in ribosome nascent chains which were extended at the $\mathrm{C}$ terminal end (Katranidis et al, 2009; Kelkar et al, 2012).

Based on the observation that polypeptide chain elongation rates on the ribosome are non-uniform (Buchan \& Stansfield, 2007) (Chapter III), it was suggested that translational pausing at certain sites along an mRNA molecule might separate distinct protein folding 
events and therefore serve as a kinetic guide for co-translational nascent chain folding on the ribosome (Komar, 2009; Purvis et al, 1987; Zhang et al, 2009). Support for this hypothesis comes from studies in which the activity or specificity of proteins was changed by the introduction of silent mutations which do not change the primary sequence of the encoded protein but are supposed to alter translation kinetics. Examples for such cases are the decreased enzymatic activities of the yeast Trp3 protein and E. coli chloramphenicol acetyltransferase after the mutation of rare codons in the corresponding genes to synonymous more frequent ones (Crombie et al, 1994; Crombie et al, 1992; Komar et al, 1999). Furthermore, a single silent mutation in the coding sequence of the multidrugresistance 1 (MDR1) gene in mammalian cells led to a change in the substrate specificity of the encoded p-glycoprotein, which was suggested to be caused by an altered conformation resulting from a change in the kinetics of co-translational protein folding (Kimchi-Sarfaty et al, 2007). Moreover, silent mutations in the gene coding for the protein Suf1 in E. coli, have been suggested to result in a different conformation of the protein making it more susceptible to limited proteolysis (Zhang et al, 2009). Altogether, these examples suggest that protein chain elongation kinetics have a critical role in coordinating co-translational folding events and might be important for the accuracy of gene expression. 


\section{AIMS OF THE THESIS}

The rate of protein biosynthesis is evolutionarily optimized with respect to accuracy of substrate selection, speed of synthesis, and possibly coordination of co-translational protein folding. The general aim of the thesis is to understand the fundamental mechanisms determining the speed and accuracy of translation and co-translational protein folding. The following questions were addressed:

- GTPase activation in EF-Tu is a critical step for the discrimination of cognate against near-cognate aa-tRNAs on the ribosome and thereby makes important contributions to translational fidelity. It is not clear why GTPase activation is impaired with near-cognate aa-tRNAs. One aim of this thesis is to understand the role of transient conformational changes of aa-tRNA in the induced fit mechanism leading to GTPase activation in EF-Tu.

- Non-uniform rates of translation elongation have been suggested to serve as a kinetic guide for sequential protein folding events on the ribosome. To directly test this hypothesis the development of methods that allow monitoring of protein elongation and folding kinetics in real time is necessary, which is the second aim of the thesis. This requires establishment of an in vitro translation system which synthesizes natural proteins with the speed and fidelity comparable to those in vivo. Furthermore, to follow folding by FRET, the co-translational incorporation of fluorescent non-canonical amino acids into nascent peptide chains needs to be optimized. 


\title{
CHAPTER I: DISTORTION OF TRNA UPON NEAR-COGNATE CODON RECOGNITION ON THE RIBOSOME ${ }^{2}$
}

\begin{abstract}
The accurate decoding of the genetic information by the ribosome relies on the communication between the decoding center of the ribosome, where the tRNA anticodon interacts with the codon, and the GTPase center of EF-Tu, where GTP hydrolysis takes place. In the $\mathrm{A} / \mathrm{T}$ state of decoding, the tRNA undergoes a large conformational change that results in a more open, distorted tRNA structure. Here we use a real-time transient fluorescence quenching approach to monitor the timing and the extent of the tRNA distortion upon reading cognate or near-cognate codons. The tRNA is distorted upon codon recognition and remains in that conformation until the tRNA is released from EF-Tu, although the extent of distortion gradually changes upon transition from the pre- to the post-hydrolysis steps of decoding. The timing and extent of the rearrangement is similar on cognate and near-cognate codons, suggesting that the tRNA distortion alone does not provide a specific switch for the preferential activation of GTP hydrolysis on the cognate codon. Thus, although the tRNA plays an active role in signal transmission between the decoding and GTPase centers, other regulators of signaling must be involved.
\end{abstract}

\section{Introduction}

Proteins are synthesized from aminoacyl-tRNAs (aa-tRNAs) that are delivered to the ribosome in ternary complexes with elongation factor Tu (EF-Tu) and GTP. The ribosome selects aa-tRNAs according to the sequence of codons in the mRNA template and rejects the bulk of aa-tRNAs with anticodons that do not match the given codon in each round of elongation. Correct base pairing between the mRNA codon and the anticodon of the tRNA on the 30S subunit of the ribosome provides a signal that is then transmitted to the GTPase center of EF-Tu on the 50S subunit and results in the activation of GTP hydrolysis by EFTu. Mismatches in the codon-anticodon complex impair GTPase activation, thereby allowing the ribosome to reject incorrect ternary complexes prior to GTP hydrolysis. Deciphering the mechanism and the specificity of signal transmission between the decoding center and the GTPase center of EF-Tu is one of the central questions in understanding the fidelity of translation.

\footnotetext{
${ }^{2}$ As published in: Mittelstaet J, Konevega AL, Rodnina MV (2011) Distortion of tRNA upon near-cognate codon recognition on the ribosome. J Biol Chem 286: 8158-8164
} 
Decoding entails a number of elemental steps. Initial binding of the ternary complex EF-Tu.GTP-aa-tRNA to the ribosome takes place codon-independently, mainly through contacts of EF-Tu with ribosomal protein L7/12, and is followed by rapid and reversible codon reading (Figure 1A; reviewed in (Rodnina et al, 2005; Rodnina \& Wintermeyer, 2001; Schmeing \& Ramakrishnan, 2009; Zaher \& Green, 2009a)).
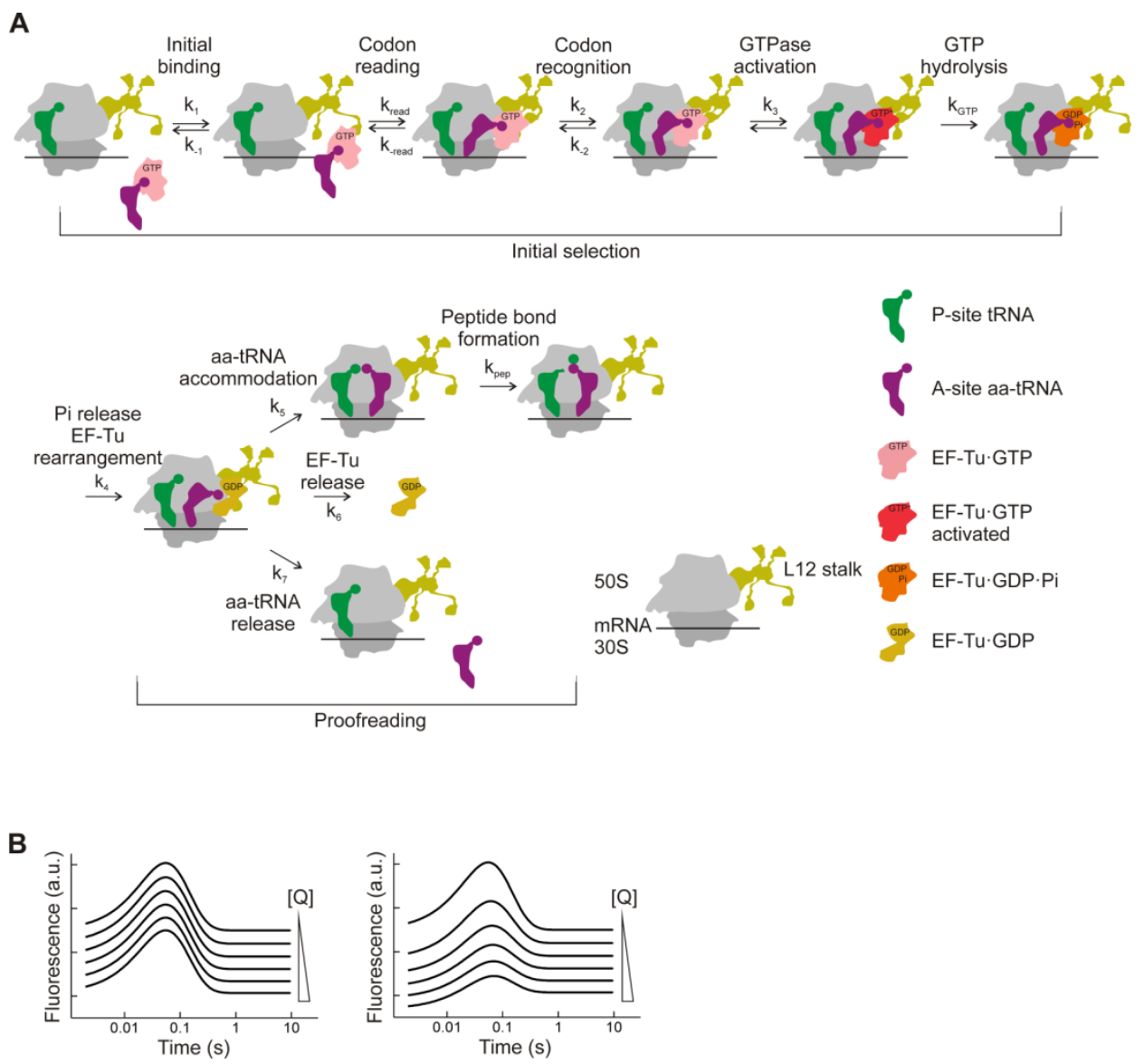

Figure 1 Experimental setup. (A) Schematic of EF-Tu-dependent aa-tRNA binding to the A site. Kinetically resolved steps are indicated by the rate constants $\mathrm{k}_{1}$ to $\mathrm{k}_{7}$ (forward reactions) and $\mathrm{k}_{-1}$ and $\mathrm{k}_{-2}$ (backward reactions). The rate of codon reading (presumably a readily reversible step (Geggier et al, 2010)) could not be determined by rapid kinetics; the values available from single-molecule FRET experiments (Blanchard et al, 2004; Geggier et al, 2010) are not comparable with the values given here due to differences in buffer conditions. Rate constants of the two chemical steps that are rate-limited by the respective preceding step are designated $\mathrm{k}_{\mathrm{GTP}}$ and $\mathrm{k}_{\mathrm{pep}}$. (B) Possible alternative outcomes of a transient fluorescence quenching experiment. Simulated time courses indicate fluorescence changes in aa-tRNA upon decoding at increasing (top to bottom) concentration of quencher (Q). Left panel: Initial, final, and transient high-fluorescence states are quenched to the same extent. Right panel: The transient intermediate is quenched more than the initial and final states.

The formation of the fully complementary codon-anticodon duplex induces local and global conformational changes at the decoding center of the ribosome, which lock the aatRNA in the codon-bound state and activate EF-Tu for rapid GTP hydrolysis (Gromadski \& Rodnina, 2004; Ogle et al, 2001; Ogle et al, 2002; Pape et al, 1999). Binding of nearcognate ternary complexes that entail single mismatches between codon and anticodon 
does not induce these structural rearrangements or rapid GTP hydrolysis, explaining why initial tRNA selection is more accurate than can be accounted for by the energetic differences between fully matched and mismatched codon-anticodon pairs alone. Hydrolysis of GTP and dissociation of inorganic phosphate (Pi) leads to a conformational rearrangement of EF-Tu which is followed by the release of aa-tRNA from EF-Tu.GDP and the dissociation of the factor from the ribosome (Kothe \& Rodnina, 2006). Aa-tRNA is then either accommodated in the peptidyl transferase center or rejected in a proofreading mechanism. Following codon recognition and prior to the release from EF-Tu, the aatRNA is bound in the so-called A/T state in which it transiently assumes a conformation that is more open or distorted compared to the unbound tRNA (Schmeing et al, 2009; Schuette et al, 2009; Stark et al, 2002; Valle et al, 2002; Villa et al, 2009; Voorhees et al, 2010). The timing or the exact step at which the tRNA changes the conformation is not known. The important role of the tRNA distortion is to pull EF-Tu into its productive, GTPase-activated conformation (Schmeing et al, 2009; Voorhees et al, 2010). It is attractive to speculate that the tRNA distortion might be a key regulator of signaling between the decoding site and the GTP binding site of EF-Tu. Mismatches in the codonanticodon complex might impair or abrogate the tRNA distortion; as a result, the GTPase conformation of EF-Tu would not be induced; hence the slow GTP hydrolysis in a nearcognate complex. In fact, the physical properties of the tRNA body are important for accurate decoding (Cochella \& Green, 2005; Ledoux et al, 2009; Piepenburg et al, 2000; Schultz \& Yarus, 1994), which would be in line with the model.

The structural details of the tRNA distortion and the concomitant rearrangements of EF-Tu that take place upon reading a correct codon on the ribosome are well-documented (Schmeing et al, 2009; Schuette et al, 2009; Stark et al, 2002; Valle et al, 2002; Villa et al, 2009; Voorhees et al, 2010). However, the conformation of the tRNA reading a nearcognate codon is not known. Here we compare the formation of the transient distorted tRNA intermediate upon reading cognate or near-cognate codons. We took advantage of a rearrangement in the D stem of aa-tRNA resulting in the $\sim 5 \AA$ displacements in the distorted tRNA in the A/T state on a cognate codon (Schmeing et al, 2009). A fluorescence reporter group, proflavin, inserted at positions $16 / 17$ in the D loop produces a fluorescent signal upon codon recognition (Rodnina et al, 1994). The effect can be explained by partial unstacking of proflavin from the neighboring guanines at positions 15 and 18 (Rodnina et al, 1994), which releases the fluorophore from static quenching (Wintermeyer \& Zachau, 1979), probably caused by photoinduced electron transfer between the fluorophore and the 
guanines (Doose et al, 2009). The distortion increases the accessibility of the proflavin reporter group for fluorescence quenchers such as iodide ions (Rodnina et al, 1994), consistent with a more open structure in the D arm region of the aa-tRNA (Schmeing et al, 2009; Schuette et al, 2009; Villa et al, 2009; Voorhees et al, 2010). Here we study the time-resolved distortion of aa-tRNA at different stages of decoding on cognate and nearcognate codons by monitoring the transient fluorescence quenching in real time.

\section{Experimental procedures}

\section{Biochemical procedures}

All experiments were carried out in buffer A (similar to HiFi (Gromadski \& Rodnina, 2004)) (50 mM Tris-HCl, pH 7.5, $50 \mathrm{mM} \mathrm{NH}_{4} \mathrm{Cl}, 50 \mathrm{mM} \mathrm{KCl}, 3.5 \mathrm{mM} \mathrm{MgCl} 2,0.5 \mathrm{mM}$ spermidine, $8 \mathrm{mM}$ putrescine, $2 \mathrm{mM} \mathrm{DTT}$ ) at $20^{\circ} \mathrm{C}$, if not stated otherwise. Titrations with fluorescence quencher were done in buffer A containing KI (suprapur grade, Merck) as indicated and $\mathrm{KCl}$, keeping the concentration of $\mathrm{KI}+\mathrm{KCl}$ constant at $150 \mathrm{mM}$. Buffer $\mathrm{B}$ (50 mM Tris- $\mathrm{HCl}, \mathrm{pH} 7.5,100 \mathrm{mM} \mathrm{NH}_{4} \mathrm{Cl}, 7 \mathrm{mM} \mathrm{MgCl}_{2}$ ) was used for the preparation of initiation and ternary complexes. Ribosomes, EF-Tu, and fMet-tRNA ${ }^{\text {fMet }}$ from E. coli were prepared as described (Gromadski \& Rodnina, 2004; Milon et al, 2007; Rodnina et al, 1994; Rodnina \& Wintermeyer, 1995). The mRNAs (28 nucleotides long) with a UUC (cognate) or CUC (near-cognate) codon following the AUG start codon were purchased from Microsynth.

The preparation of proflavin-labeled yeast tRNA ${ }^{\text {Phe }}$ proceeds in two steps: reduction of the dihydroU base at position $16 / 17$ in the $\mathrm{D}$ loop by borohydride treatment followed by the attachment of proflavin (Wintermeyer \& Zachau, 1979). tRNA ${ }^{\text {Phe }}\left(10 \mathrm{~A}_{260}\right.$ units/ml in $0.2 \mathrm{M}$ Tris- $\mathrm{HCl}(\mathrm{pH} 7.5)$ ) was mixed with $\mathrm{NaBH}_{4}$ solution (100 mg in $\left.1 \mathrm{ml} \mathrm{KOH}\right)$. After incubation for $30 \mathrm{~min}$ at $0^{\circ} \mathrm{C}$ in the dark, the reaction was stopped by the addition of acetic acid to $\mathrm{pH} 4-5$, and the tRNA was precipitated with cold ethanol and $0.3 \mathrm{M}$ potassium acetate ( $\mathrm{pH}$ 4.5). Ethanol precipitation was repeated 3-4 times to remove traces of borohydride. Proflavin labeling was carried out by adding borohydride-treated tRNA ${ }^{\text {Phe }}$ to $3 \mathrm{mM}$ proflavin in $0.1 \mathrm{M}$ sodium acetate $\mathrm{pH} 4.3$. After incubation for $2 \mathrm{~h}$ at $37^{\circ} \mathrm{C}$ in the dark, the reaction was stopped by the addition of $1 \mathrm{M}$ Tris- $\mathrm{HCl}(\mathrm{pH}$ 9) to $\mathrm{pH} 7.5$. Free dye was removed by phenol extraction and ethanol precipitation. Incorporation of proflavin was quantified photometrically by measuring optical density at $260 \mathrm{~nm}$ and $460 \mathrm{~nm}$. For fully labeled tRNA, the $\mathrm{A}_{460}: \mathrm{A}_{260}$ ratio is 0.055 . 
Initiation complexes were formed in buffer B by incubating ribosomes $(1 \mu \mathrm{M})$, mRNA $(3 \mu \mathrm{M}), \mathrm{f}\left[{ }^{3} \mathrm{H}\right] \mathrm{Met}^{-t R N A}{ }^{\mathrm{fMet}}(1.5 \mu \mathrm{M}), \mathrm{IF} 1, \mathrm{IF} 2, \mathrm{IF} 3(1.5 \mu \mathrm{M}$ each), and GTP $(1 \mathrm{mM})$ for $1 \mathrm{~h}$ at $37^{\circ} \mathrm{C}$, and purified by centrifugation through a $1.1 \mathrm{M}$ sucrose cushion in buffer $\mathrm{B}$ $(400 \mu \mathrm{l})$ for $2 \mathrm{~h}$ at 259,000 x $g$ in a Beckmann Optima Max-XP ultracentrifuge at $4^{\circ} \mathrm{C}$. After centrifugation, pellets were dissolved in buffer B, shock-frozen in liquid nitrogen and stored at $-80^{\circ} \mathrm{C}$. Ternary complex EF-Tu.GTP. $\left[{ }^{14} \mathrm{C}\right] \mathrm{Phe}-\mathrm{tRNA}{ }^{\mathrm{Phe}}(\mathrm{Prf})$ was prepared by incubating EF-Tu (wild-type or H84A mutant) $(50 \mu \mathrm{M})$, GTP $(1 \mathrm{mM})$, phosphoenol pyruvate $(3 \mathrm{mM})$, pyruvate kinase $(0.05 \mathrm{mg} / \mathrm{ml}), \operatorname{tRNA}^{\text {Phe }}(\operatorname{Prf})(25 \mu \mathrm{M}), \mathrm{CTP}(1 \mathrm{mM})$, ATP $(3 \mathrm{mM}),\left[{ }^{14} \mathrm{C}\right]$-phenylalanine $(40 \mu \mathrm{M})$, nucleotidyl-terminal transferase and phenylalanyl-tRNA synthetase $(0.5 \% \mathrm{v} / \mathrm{v}$ each $)$ in buffer B containing $20 \mathrm{mM} \mathrm{MgCl}_{2}$ and purified by gel filtration on 2xSuperdex $75 \mathrm{HR}$ columns (GE Healthcare) in buffer B. Ternary complex and initiation complexes were adjusted to buffer A immediately before the experiments.

\section{Kinetic measurements}

Fluorescence stopped-flow experiments were performed using a SX-20MV apparatus (Applied Photophysics, Leatherhead, UK), monitoring proflavin fluorescence. Excitation was at $463 \mathrm{~nm}$ and the fluorescence was measured after passing a 500-nm cutoff filter (KV 500, Schott). Time courses were measured at pseudo-first-order conditions in excess of initiation complexes $(1 \mu \mathrm{M})$ over ternary complexes $(0.2 \mu \mathrm{M})$ and were evaluated by fitting an exponential function, $\mathrm{F}=\mathrm{F}_{\infty}+\mathrm{A} \times \exp \left(-\mathrm{k}_{\mathrm{app}} \times \mathrm{t}\right)$. If necessary, additional exponential terms were included. The differential amplitudes obtained at different KI concentrations were fitted according to the Stern-Volmer equation in the form $A_{0}-A=A_{0}$ $\times\left(1-1 /\left(1+\mathrm{K}_{\mathrm{SV}} \times[\mathrm{KI}]\right)\right)$ to yield Stern-Volmer constants, $\mathrm{K}_{\mathrm{SV}}$, for collisional quenching. Steady-state fluorescence measurements were carried out in a Fluorolog-3 (Horiba Jobin Yvon) spectrofluorimeter. Excitation was at $463 \mathrm{~nm}$ and the emission was measured at 502 $\mathrm{nm}$; the $\mathrm{K}_{\mathrm{SV}}$ values were calculated as described (Robertson \& Wintermeyer, 1981). Calculations were performed using TableCurve (Jandel Scientific) or Prism (Graphpad Software). Modeling of reaction intermediates was performed in Scientist (Micromath) based on the following kinetic scheme:

$$
\begin{aligned}
& \begin{array}{lllll}
k_{1} & k_{2} & k_{3} & k_{4} & k_{5}
\end{array} \\
& I C+T C \underset{k_{-1}}{\stackrel{k_{-2}}{\leftrightarrows}} A \underset{k_{7}}{\leftrightarrows} B \rightarrow C \underset{F}{D} \underset{ }{\leftrightarrows} E
\end{aligned}
$$


where ternary complex (TC) and initiation complex (IC) form the initial binding complex A, which is converted to codon recognition complex B. C represents the state after GTPase activation and GTP hydrolysis, D the state after phosphate release from EF-Tu, and E the state after accommodation of the aa-tRNA into the A site. F represents the proofreading pathway, in which aa-tRNA is rejected from the ribosome. For the cognate ternary complex, the following rate constants were used: $\mathrm{k}_{1}=140 \mu \mathrm{M}^{-1} \mathrm{~s}^{-1}$ and $\mathrm{k}_{-1}=85 \mathrm{~s}^{-1}$ for initial binding, $\mathrm{k}_{2}=190 \mathrm{~s}^{-1}$ and $\mathrm{k}_{-2}=0.23 \mathrm{~s}^{-1}$ for codon recognition, $\mathrm{k}_{3}=260 \mathrm{~s}^{-1}$ for GTPase activation and GTP hydrolysis and $\mathrm{k}_{5}=23 \mathrm{~s}^{-1}$ for the accommodation and peptide bond formation (Gromadski \& Rodnina, 2004). The rate of Pi release $\left(\mathrm{k}_{4}=10 \mathrm{~s}^{-1}\right.$, data not shown) was measured for the present conditions as described (Kothe \& Rodnina, 2006). For the near-cognate ternary complexes, $\mathrm{k}_{1}=140 \mu \mathrm{M}^{-1} \mathrm{~s}^{-1}, \mathrm{k}_{-1}=85 \mathrm{~s}^{-1}, \mathrm{k}_{2}=190 \mathrm{~s}^{-1}, \mathrm{k}_{-2}=80$ $\mathrm{s}^{-1}$, and $\mathrm{k}_{3}=0.1 \mathrm{~s}^{-1}$ were used ((Gromadski \& Rodnina, 2004) and data not shown). The $\mathrm{k}_{5}$ and $\mathrm{k}_{7}$ values for the accommodation and rejection of the near-cognate tRNA could not be determined at HiFi conditions, because the GTPase activation is strongly rate-limiting. Because the efficiency of proofreading is essentially the same in HiFi (Gromadski \& Rodnina, 2004) and the buffer with $10 \mathrm{mM} \mathrm{Mg}^{2+}$ (Pape et al, 1999), the values from the latter work were used, $\mathrm{k}_{5}=0.1 \mathrm{~s}^{-1}$ and $\mathrm{k}_{7}=6 \mathrm{~s}^{-1}$. The concentration of IC and TC used for modeling were the same as in the biochemical experiments, $1 \mu \mathrm{M}$ and $0.2 \mu \mathrm{M}$, respectively. 


\section{Results}

\section{Transient fluorescence quenching approach}

To assess the extent of tRNA distortion at the D loop, the binding of the ternary complex EF-Tu.GTP.Phe-tRNA ${ }^{\text {Phe }}(\operatorname{Prf})$ to the ribosome was followed in a stopped-flow apparatus, monitoring proflavin fluorescence in the presence of increasing concentrations of the fluorescence quencher KI, while keeping the ionic strength constant. Changes of proflavin fluorescence report the transient formation of several intermediates of decoding (Pape et al, 1998; Rodnina et al, 1994). If the fluorescence in all intermediates were quenched to the same extent, then the relative amplitudes of the various kinetic steps would be expected to be the same in the presence or the absence of the quencher (Figure 1B). Alternatively, if the fluorescence of a certain intermediate would be quenched more than that of other states, this would indicate a higher exposure of the fluorophore and hence a more open tRNA conformation in that intermediate. To determine the quenching constant of an intermediate, the time courses obtained in the presence of various concentrations of $\mathrm{KI}$ (denoted as I) are subtracted from the one obtained without quencher $\left(\mathrm{I}_{0}\right)$. The resulting differential curves $\left(\mathrm{I}_{0}-\mathrm{I}\right)$ can be deconvoluted into exponential terms which are characterized by the apparent rate constants $\left(\mathrm{k}_{\mathrm{app}}\right)$ and amplitudes $\left(\mathrm{A}_{0}-\mathrm{A}\right)$ of the respective steps. To determine the Stern-Volmer quenching constant, $\mathrm{K}_{\mathrm{SV}}$, which depends on the accessibility of the fluorophore for the quencher and, therefore, is a measure for the "openness" of the tRNA, the differential amplitudes of each step were plotted against the concentration of KI, and the plots were evaluated according to the Stern-Volmer relationship (Methods). The transient fluorescence quenching approach is particularly suitable for the analysis of transient intermediates in rapid, forward-committed reactions, such as the EF-Tu-dependent aa-tRNA binding to the A site. Other advantages of the transient quenching approach are the possibilities (i) to isolate a distorted tRNA intermediate from the coexisting ensemble of states that are not distorted as the reaction proceeds and (ii) to selectively monitor those tRNAs that bind to the ribosome, because only those contribute to fluorescence changes.

\section{Transient distortions}

We first monitored the changes of the tRNA conformation that take place upon reading a cognate codon. The ternary complex, EF-Tu.GTP.Phe-tRNA ${ }^{\text {Phe }}(\operatorname{Prf})$, was mixed with the ribosomal initiation complex, 70S-mRNA.fMet-tRNA ${ }^{\mathrm{fMet}}$ exposing a cognate UUC codon in the A site and the changes in Prf fluorescence were monitored (Figure 2A). 
As observed previously, the proflavin fluorescence transiently increased during the time course of the reaction. According to our previous detailed step assignment, the fluorescence increase reflects all steps starting from ternary complex binding to the ribosome up to GTPase activation (Gromadski \& Rodnina, 2004; Pape et al, 1998; Rodnina et al, 1994) (Figure 1A). The decrease in fluorescence coincides with Pi release from EF-Tu following GTP hydrolysis, the release of the aa-tRNA from EF-Tu, and the subsequent accommodation of the aa-tRNA in the A site (Figure 1A).
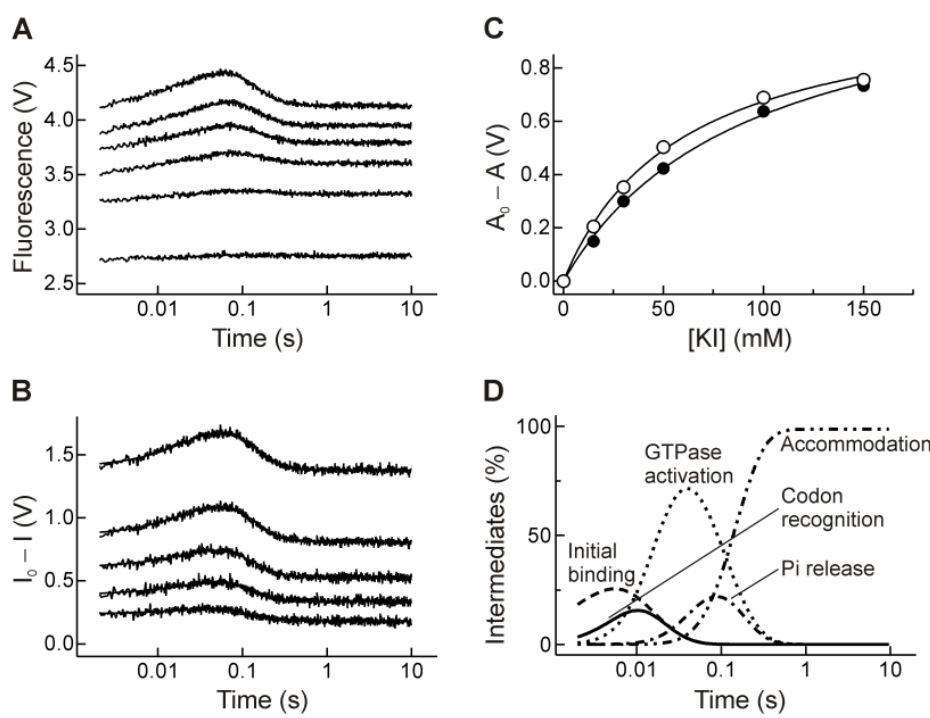

Figure 2 Transient distortion of Phe-tRNA ${ }^{\text {Phe }}$ (Prf) upon ternary complex binding to ribosomes with a cognate UUC codon in the A site. (A) Fluorescence change at increasing KI concentrations (top to bottom: 0, 15, 30, 50, 100, and $150 \mathrm{mM} \mathrm{KI).} \mathrm{(B)} \mathrm{Deconvolution} \mathrm{of} \mathrm{intermediates.} \mathrm{Differential} \mathrm{curves} \mathrm{obtained} \mathrm{by} \mathrm{the}$ subtraction of the quenched traces (indicated as I) from the trace measured in absence of quencher $\left(\mathrm{I}_{0}\right)$ (top to bottom: $\mathrm{I}_{0}-\mathrm{I}$ at $[\mathrm{KI}]=150,100,50,30$, and $15 \mathrm{mM}$ ). Solid lines show two-exponential fits with $\mathrm{k}_{\mathrm{app} 1}=30 \mathrm{~s}^{-}$ ${ }^{1}$ and $\mathrm{k}_{\mathrm{app} 2}=10 \mathrm{~s}^{-1}$. (C) Stern-Volmer plots for quenching during the early (codon recognition, GTPase activation, GTP hydrolysis (Figure 1A); closed circles) and late (post-hydrolysis steps of Figure. 1A; open circles) steps of ternary complex binding. The data were analyzed using the Stern-Volmer equation (Methods) and the results are summarized in Table 1. (D) Evolution of intermediate states of decoding. Time courses were modeled as described in Methods. The intermediates indicated are states after: Initial binding (dashed line); codon recognition (solid line); GTPase activation and GTP hydrolysis (dotted line); Pi release and conformational change of EF-Tu (dash-dot line); accommodation and peptide bond formation (dash-dotdot).

When the stopped-flow experiments were carried out in the presence of KI, the fluorescence of Phe-tRNA ${ }^{\text {Phe }}(\operatorname{Prf})$ in both initial and final states was decreased due to quenching. In comparison, the fluorescence of the transient intermediate was decreased to a larger extent, indicating a higher accessibility of the fluorophore for the quencher and thus a more open tRNA intermediate (the scenario depicted in the right panel of Figure 1B). Notably, at the highest KI concentration used, the transient fluorescence increase was no longer observed, indicating complete quenching and suggesting that the majority of the aa-tRNA assumes the distorted, more open state during the decoding of a cognate codon (see amplitudes in Table 1). The differential curves could be accurately evaluated with a 
two-exponential function, accounting for the distortion of the tRNA and the relaxation back into the undistorted conformation (Figure $2 \mathrm{~B}$ ). The $\mathrm{K}_{\mathrm{SV}}$ values determined from the amplitudes of the distortion and relaxation steps (Figure 2C) were in the range of 11$17 \mathrm{M}^{-1}$, much higher than the value of $5 \mathrm{M}^{-1}$ obtained for the tRNA free in solution or in the ternary complex with EF-Tu.GTP (Table 1). Given that all individual rate constants are known (Gromadski et al, 2006; Gromadski \& Rodnina, 2004), the elemental step (Figure 1A) can be identified at which tRNA changes the conformation (Figure 2D). The formation of the distorted intermediate proceeds with the same rate as codon recognition, about $30 \mathrm{~s}^{-1}$ at the ligand concentrations used.

Table 1 Distortion of Phe-tRNA ${ }^{\text {Phe }}($ Prf) upon decoding of cognate and near-cognate codons

\begin{tabular}{lllll}
\hline \multirow{2}{*}{ State } & \multicolumn{2}{c}{ Cognate } & \multicolumn{2}{c}{ Near-cognate } \\
\cline { 2 - 5 } & $\mathrm{K}_{\mathrm{SV}}, \mathrm{M}^{-1}$ & $\mathrm{~A}_{0}-\mathrm{A}$ & $\mathrm{K}_{\mathrm{SV}}, \mathrm{M}^{-1}$ & $\mathrm{~A}_{0}-\mathrm{A}$ \\
\hline No ribosome $^{\mathrm{a}}$ & $5.4 \pm 0.2$ & n.a. & $5.4 \pm 0.2$ & n.a. \\
Initial binding $^{\mathrm{b}}$ & $7 \pm 2$ & 0.32 & $7 \pm 2$ & 0.32 \\
Early steps $^{\mathrm{c}}$ & $11 \pm 1$ & 1.00 & $14 \pm 2$ & 0.56 \\
Late steps $^{\mathrm{c}}$ & $17 \pm 1$ & 1.00 & $14 \pm 2$ & 0.08 \\
Stalled by EF-Tu(H84A) & $10 \pm 1$ & 0.46 & $11 \pm 2$ & 0.30 \\
Stalled by kirromycin & $16 \pm 4$ & 0.44 & n.d. ${ }^{\mathrm{d}}$ & n.d. $^{\text {d }}$ \\
\hline
\end{tabular}

${ }^{\mathrm{a}} \mathrm{K}_{\mathrm{SV}}$ in the absence of ribosomes was measured for the ternary complex EF-Tu.GTP.Phe-tRNA ${ }^{\text {Phe }}($ Prf) at steady-state conditions (Robertson \& Wintermeyer, 1981); the calculation of the transient differential amplitudes $\mathrm{A}_{0}-\mathrm{A}$ is not applicable (n.a.). The $\mathrm{K}_{\mathrm{SV}}$ values for the free $\mathrm{tRNA}^{\mathrm{Phe}}(\mathrm{Prf})$ and free proflavin are 5.3 $\pm 0.1 \mathrm{M}^{-1}$ and $70 \pm 1 \mathrm{M}^{-1}$, respectively.

b $\mathrm{K}_{\mathrm{SV}}$ value for the initial binding complex was measured in a model system with non-programmed ribosomes (Rodnina et al, 1996)

${ }^{c}$ Early steps include pre-hydrolysis and GTP hydrolysis intermediates; late steps reflect post-hydrolysis steps (Figure 1A). Distribution of intermediates is shown in Figs. 2D and 3D.

${ }^{\mathrm{d}}$ n.d., complex not detectable

The distorted intermediate accumulates through the early steps of decoding, i.e. codon recognition, GTPase activation, and GTP hydrolysis (Figure 1A). The tRNA relaxation takes place at the same rate, about $10 \mathrm{~s}^{-1}$, as the steps following GTP hydrolysis (Figure 1A), which in the following are collectively denoted as the "late steps" of decoding, in contrast to the "early steps" at which the distorted tRNA intermediate is formed. Thus, the tRNA is distorted upon codon recognition and remains in an open conformation through the pre- and post-hydrolysis steps (Figure 1A) until it is released from EF-Tu. On the other hand, the difference in the degree of tRNA distortion at the early and late states $\left(\mathrm{K}_{\mathrm{SV}}\right.$ values of 11 vs. $17 \mathrm{M}^{-1}$ ) may suggest that the structural details of the conformational rearrangement gradually change upon transition from the early to the late steps of decoding (Figure 2C and Table 1). When analogous experiments were carried out with ribosome complexes exposing a near-cognate CUC codon, a fluorescence increase of PhetRNA $^{\text {Phe }}($ Prf) was observed followed by a very slow decrease (Figure 3A). Previous 
analysis suggested that most of the amplitude of the fluorescence increase is due to the formation of the codon-recognition complex ((Gromadski \& Rodnina, 2004) and below). Subsequent GTPase activation is impaired and GTP hydrolysis proceeds with a rate of about $0.1 \mathrm{~s}^{-1}$, which is rate-limiting for the following steps of accommodation and proofreading that are observed as a fluorescence decrease (Figure 3A). Because of slow GTPase activation, the codon-recognition complex accumulates as a high-fluorescence intermediate, and the addition of KI predominantly decreases the fluorescence of that intermediate (Figure 3B).
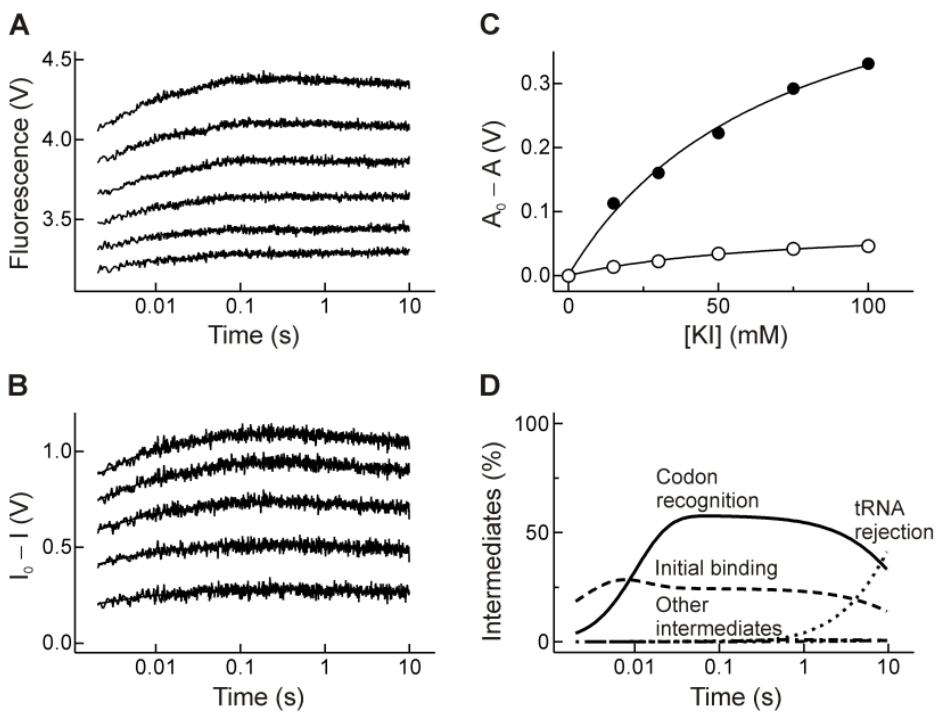

Figure 3 Transient distortion of Phe-tRNA ${ }^{\text {Phe }}(\mathrm{Prf})$ upon ternary complex binding to ribosomes with a nearcognate CUC codon in the A site. (A) Fluorescence change at increasing KI concentrations (top to bottom: 0, 15, 30, 50, 75, and $100 \mathrm{mM} \mathrm{KI}$ ). (B) Deconvolution of intermediates as described in Figure 2B. (C) SternVolmer plots for quenching during the early (closed circles) and late (open circles) steps of ternary complex binding. The data were analyzed using the Stern-Volmer equation (Methods) and the results are summarized in Table 1. (D) Evolution of intermediate states of decoding. Time courses were modeled as described in Methods. The intermediates indicated are states after: Initial binding (dashed line); codon recognition (solid line); and tRNA rejection (dotted line). The intermediates after GTPase activation and GTP hydrolysis (dotted line); Pi release and conformational change of EF-Tu (dash-dot line); accommodation and peptide bond formation (dash-dot-dot) do not accumulate.

As determined from the KI dependence of the differential amplitudes (Figure 3C), the $\mathrm{K}_{\mathrm{SV}}$ values of the early and late steps are $14 \mathrm{M}^{-1}$ (Table 1 ), which indicates the formation of an open tRNA intermediate also when the codon-anticodon complex contains a mismatch. Thus, upon reading a near-cognate codon, tRNA is distorted at the same step (upon codon recognition) and to an extent similar to that on a cognate codon.

Kinetic modeling based on the known kinetic constants (Gromadski \& Rodnina, 2004) suggests that $53 \%$ of the ribosome-bound tRNA is in the codon-recognition complex (Figure 3D), which is in excellent agreement with the $56 \%$ fluorescence amplitude of the intermediate that is strongly quenched by KI (Table 1). From the distribution of the 
decoding intermediates, about $25 \%$ of aa-tRNA remains in the initial binding complex throughout the reaction (Figure 3D), which may reduce the overall $\mathrm{K}_{\mathrm{SV}}$ value observed for an ensemble represented by a mixture of states. Therefore, the true value of $\mathrm{K}_{\mathrm{SV}}$ of either early or late steps is likely to be even higher than $14 \mathrm{M}^{-1}$. This would suggest that the tRNA distortion in the near-cognate pre-hydrolysis state may be even more extensive than in the cognate case (>14 $\mathrm{M}^{-1}$ compared to $11 \mathrm{M}^{-1}$ ). The $\mathrm{K}_{\mathrm{SV}}$ values of the post-hydrolysis states are not significantly different $\left(>14 \mathrm{M}^{-1}\right.$ and $17 \mathrm{M}^{-1}$ on near-cognate and cognate codons, respectively), suggesting that the conformation of the tRNA is similar in those states.

\section{Conformation of the tRNA in the isolated intermediates}

To further substantiate the assignment of early and late states, we have chosen conditions at which several intermediates of A-site binding can be stalled. As a model for the initial, codon-independent binding complex, we have used EF-Tu.GTP.PhetRNA $^{\text {Phe }}$ (Prf) bound to vacant ribosomes (Rodnina et al, 1996). The $K_{S v}$ value of that complex, as determined by transient fluorescence quenching, $7 \mathrm{M}^{-1}$ (Table 1) is in excellent agreement with the value determined at steady-state conditions (MVR, unpublished data). The value is not much different from that of unbound or EF-Tu-bound tRNA, suggesting that in the initial binding complex the tRNA preferentially assumes a non-distorted conformation.

To stall the codon-recognition complex, GTP hydrolysis had to be inhibited. For this purpose, we utilized a mutant of EF-Tu which was impaired in GTP hydrolysis by replacing the catalytic His84 with Ala (Daviter et al, 2003). Upon binding to the cognate codon, the ternary complex EF-Tu(H84A) GTP.Phe-tRNA ${ }^{\text {Phe }}$ proceeds through all steps up to codon recognition with rates similar to the wild-type complex, but is stalled prior to GTP hydrolysis (Daviter et al, 2003) (Figure 4A). The GTPase-activated state may be transiently sampled in this complex (Daviter et al, 2003), but cannot be stabilized by the interactions of His84 with the sarcin-ricin loop of the ribosome, as seen with the wild-type EF-Tu (Voorhees et al, 2010), and therefore does not accumulate. The overall amplitude of the early steps of decoding appears smaller with mutant compared to the wild type EF-Tu, (Figure 4A); this can be explained by small structural differences between the A/T complexes in the pre- and post-hydrolysis states (Schmeing et al, 2009; Voorhees et al, 2010). The $\mathrm{K}_{\mathrm{SV}}$ value of the pre-hydrolysis state, as determined by the transient fluorescence quenching approach, is about $10 \mathrm{M}^{-1}$, which again indicates the aa-tRNA 
conformation that is more open than in the ternary complex or in free tRNA. The value is similar to that estimated for the early steps, but is somewhat lower than for the late steps of the uninterrupted decoding with wild-type EF-Tu. The contribution of the undistorted initial binding state should be relatively small in this case, as the tRNA is predominantly present in the codon-recognition state (Figure 4B). This suggests that the $\mathrm{K}_{\mathrm{SV}}=10 \mathrm{M}^{-1}$ reflects the distortion of aa-tRNA during decoding of a cognate codon.
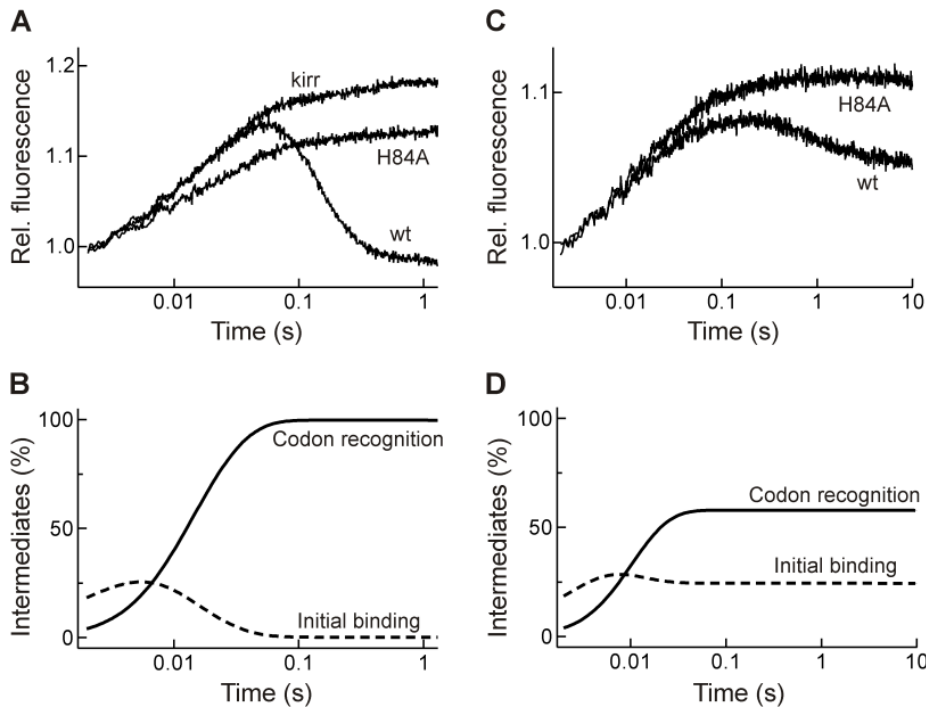

Figure 4 Isolation of early and late decoding intermediates. (A) Stalling the A/T state of cognate tRNA using mutant EF-Tu(H84A) or kirromycin (kirr); wt, wild-type EF-Tu. (B) Modeled distribution of tRNA intermediates upon decoding the cognate codon in the presence of EF-Tu(H84A). The intermediates indicated are: Initial binding (dashed line) and codon recognition (solid line); GTP hydrolysis is abolished. (C) Stalling of the near-cognate A/T state using EF-Tu(H84A). (D) Modeled distribution of the tRNA intermediates with EF-Tu(H84A) upon decoding a near-cognate codon. Intermediates are as indicated in (B).

When a near-cognate codon was used, the aa-tRNA bound in the A/T complex with EF$\mathrm{Tu}(\mathrm{H} 84 \mathrm{~A})$ is also distorted (Figure $4 \mathrm{C}$ ); the $\mathrm{K}_{\mathrm{Sv}}$ value of $11 \mathrm{M}^{-1}$ is similar to that in the cognate complex (Table 1). However, because in the near-cognate case about $25 \%$ of aatRNA remains in the initial binding step (Figure 4D), which is characterized by a lower $\mathrm{K}_{\mathrm{SV}}$, the true $\mathrm{K}_{\mathrm{SV}}$ value for the near-cognate codon-recognition complex is likely $>11 \mathrm{M}^{-1}$. The data suggest that in the pre-hydrolysis complex the tRNA assumes a distorted conformation, regardless of whether the A-site codon is cognate or near-cognate.

Finally, for better comparison with the structures of the complexes obtained by cryoEM and crystallography, we also determined the $\mathrm{K}_{\mathrm{Sv}}$ value for aa-tRNA stalled in the A/T state by kirromycin. The antibiotic does not affect the early steps of decoding up to GTP hydrolysis and Pi release from EF-Tu, but blocks the conformational change of EF-Tu that leads to the release of aa-tRNA from the factor (Kothe \& Rodnina, 2006; Rodnina et al, 1994). In this state, the cognate aa-tRNA is strongly distorted with a $\mathrm{K}_{\mathrm{SV}}=16 \mathrm{M}^{-1}$ (Table 
1), consistent with the value obtained for the late decoding state in full decoding. This finding further substantiates the notion that the conformation of the tRNA gradually changes towards a more open structure upon progressing from the pre- to the posthydrolysis states. As kirromycin did not stabilize the binding of aa-tRNA on the nearcognate codon (data not shown), analogous experiments with the near-cognate A/T state were not feasible.

\section{Discussion}

\section{The timing of tRNA distortion}

The present results suggest the following timing of the conformational rearrangements of aa-tRNA during decoding. In the ternary complex bound to the ribosome before codon reading (Figure 1), aa-tRNA retains its undistorted, closed conformation, although transient excursions into an open conformation may occur. Upon cognate codon recognition, the tRNA assumes a conformation which is significantly more open at the D loop than in the preceding initial binding complex or in the free ternary complex with EFTu.GTP (Table 1). The solvent exposure of the D loop increases even further in the posthydrolysis state up to the step when the tRNA is released from EF-Tu and accommodated in the A site (Figure 1), which allows the tRNA to relax into the undistorted conformation. The gradual change in the tRNA arrangement between the early and late steps of decoding may reflect structural differences between the pre- and post-hydrolysis states, although the crystal structures of the tRNA in the two states are very similar (Schmeing et al, 2009; Voorhees et al, 2010). Alternatively, as the codon-recognition step presumably entails a number of substeps and the tRNA rapidly samples between different conformations and substates (Geggier et al, 2010; Ogle et al, 2001; Ogle et al, 2002), the observed solvent exposure of the given state may be a global value that represents a mixture of undistorted and distorted states. The open tRNA conformation may be favored by the contacts with the ribosome as soon as codon recognition takes place and may be further stabilized in the post-hydrolysis state.

\section{Distortion of tRNA in the near-cognate A/T state}

The tRNA plays an important role in activation of GTP hydrolysis. An intact aa-tRNA is required for the GTPase activation of EF-Tu (Piepenburg et al, 2000) and mutations in the D arm affect GTP hydrolysis (Cochella \& Green, 2005; Pan et al, 2008). The structures suggest how the tRNA distortion on a cognate codon may result in the GTPase activation 
of EF-Tu (Schmeing et al, 2009; Voorhees et al, 2010). This raises the question whether the tRNA distortion provides a switch that signals the formation of a correctly matched cognate codon-anticodon complex to the GTPase center. In the simplest case, a mismatch would impair the formation of the distorted tRNA intermediate, thus precluding the structural rearrangements that are induced by cognate codon recognition and are required for GTPase activation of EF-Tu. The present data demonstrate that the formation of the open tRNA intermediate does not depend on cognate codon recognition (Table 1): When a near-cognate codon is recognized, the tRNA is distorted as well, and the timing of the rearrangements is similar to the one on the cognate codon; yet GTP hydrolysis is more than 2000-fold slower in the near-cognate compared to the cognate complex. Thus, the tRNA distortion alone does not seem to provide the specific signal for the preferential activation of GTP hydrolysis by EF-Tu in the cognate case. Additional regulators, presumably ribosome elements, must play a role in sensing and transmitting the signals that communicate the decoding to GTPase activation (see below).

In a more complicated scenario the details of the distortion may be different on the cognate and near-cognate codons, thereby affecting the GTPase activation in different ways. Given the similarity of the quenching constants of the distorted intermediates formed on cognate and near-cognate codons, large differences in the respective tRNA conformations seem unlikely, although small differences cannot be excluded. The D loop of the tRNA bound on the near-cognate codon may be even slightly more open than on the cognate one (see Results). Structural differences in tRNA regions distant from the D loop cannot be ruled out, but seem unlikely, given the rigidity of the molecule and the coupling in rearrangements of the tRNA elbow region and the acceptor stem interacting with EF-Tu (Sanbonmatsu, 2006; Schmeing et al, 2009; Whitford et al, 2010).

\section{Consequences for GTP hydrolysis}

GTP hydrolysis proceeds through the attack of the hydrolytic water molecule on the $\gamma$ phosphate of GTP in EF-Tu. His84 in Escherichia coli EF-Tu is the active site residue that stabilizes the GTPase transition state (Berchtold et al, 1993; Daviter et al, 2003; Voorhees et al, 2010). Upon GTPase activation on the ribosome, His84 has to move towards the $\gamma$ phosphate, and this movement should be induced only when a correct codon-anticodon complex is formed. The tRNA distortion affects the relative orientation between tRNA and EF-Tu (Schmeing et al, 2009; Schuette et al, 2009; Villa et al, 2009; Voorhees et al, 2010), which leads to subtle rearrangement in EF-Tu and stabilization of the catalytically active 
orientation of His84 by A2662 of the sarcin-ricin loop of 23S rRNA (Voorhees et al, 2010), thereby ultimately resulting in GTPase activation. The mechanism of activation must be precisely tuned for each cognate aa-tRNA, as all of them exhibit similar kinetic properties despite a wide variety of structural features (Ledoux \& Uhlenbeck, 2008). Similarly, any mismatch in the codon-anticodon complex impairs GTPase activation, regardless of the thermodynamic stability of the respective codon-anticodon complexes or their docking partners at the decoding site (Gromadski et al, 2006). The uniformity of mismatch recognition suggests a global response mechanism, which would be consistent with the idea that all conformational changes that occur upon cognate codon recognition, including domain closure of the $30 \mathrm{~S}$ subunit, distortions of the tRNA, and rearrangements in EF-Tu, are essential for the precise positioning of the GTPase center of EF-Tu at the sarcin-ricin loop. Although the tRNA is distorted also in the near-cognate A/T state, even subtle changes in the orientation of tRNA and EF-Tu could cause defects in the GTPase activation by preventing A2662 from properly placing His84 into the active site (Voorhees et al, 2010). In this framework, the tRNA mutants that activate GTP hydrolysis on a nearcognate codon (Cochella \& Green, 2005; Ledoux et al, 2009) appear to have found their own unique conformational solution to dock EF-Tu on the sarcin-ricin loop. However, other contacts in the decoding complex may specifically affect the stringency of decoding, e.g. helix 14 and helix 8 of 16S rRNA that negatively regulate GTP hydrolysis (McClory et al, 2010), or the interactions between helix 5 and domain 2 of EF-Tu (Vorstenbosch et al, 1996). The structural basis for the very strong effect of ribosomal protein L7/12 on the GTPase activity of EF-Tu (Kothe et al, 2004; Mohr et al, 2002) remains to be clarified. Finally, the ribosome may play an active role in monitoring the correct codon-anticodon interaction using a network of rRNA and proteins from both ribosomal subunits, as suggested by the recent crystal structure of the proofreading complex (Jenner et al, 2010a). Further experiments will be necessary to determine the role of each interaction element in the $\mathrm{A} / \mathrm{T}$ state in the codon-specific control of the GTPase activation of EF-Tu. 


\title{
CHAPTER II: KINETIC ANALYSIS OF THE INCORPORATION OF A FLUORESCENT NON-CANONICAL AMINO ACID ON THE RIBOSOME
}

\begin{abstract}
Non-canonical amino acids have a multitude of applications in synthetic biology. The site-specific incorporation of fluorescent amino acids is of particular interest in studies of protein folding and protein-ligand interactions. Many reports have shown the successful incorporation of fluorescent amino acids into proteins. However, little is known about the factors that determine the efficiency and speed of non-canonical amino acid incorporation on the ribosome. Here the incorporation of a fluorescent non-canonical amino acid $\left(\varepsilon \mathrm{NH}_{2}-\right.$ Bodipy576/589-Lysine) was analyzed using pre-steady state kinetic approaches. While the binding of $\varepsilon \mathrm{NH}_{2}$-Bodipy576/589-Lys-tRNA ${ }^{\text {Lys }}$ to EF-Tu is strongly affected by the presence of the modified amino acid, the incorporation of the non-canonical amino acid on the ribosome occurs with comparable speed and efficiency as for canonical lysine. By monitoring the dissociation of $\varepsilon \mathrm{NH}_{2}$-Bodipy576/589-Lys-tRNA ${ }^{\mathrm{Lys}}$ from EF-Tu and its accommodation on the ribosome, using a time-resolved FRET assay, the trajectory of noncanonical aa-tRNA incorporation on the ribosome was resolved. We find that the accommodation of $\varepsilon \mathrm{NH}_{2}$-Bodipy576/589-Lys-tRNA ${ }^{\text {Lys }}$ is not significantly delayed at the accommodation gate of the ribosome and that peptide bond formation is not rate-limiting the incorporation of the non-canonical amino acid. Altogether, this study emphasizes the role of the quality control mechanism of aa-tRNA recognition by EF-Tu as a bottleneck for the incorporation of non-canonical amino acids into proteins but also provides conditions for an efficient incorporation of non-canonical amino acids on the ribosome.
\end{abstract}

\section{Introduction}

The genetic code is highly redundant, with 64 base triplets coding for only 20 different standard amino acids and the termination of polypeptide synthesis. Furthermore, 2 nonstandard proteinogenic amino acids, selenocysteine and pyrrolysine, can be encoded by the stop codons UGA and UAG, but require an additional mRNA sequence which forms a structural element downstream of the insertion site called SECIS and PYLIS, respectively (Driscoll \& Copeland, 2003; Krzycki, 2005). The extension of the genetic code beyond the standard canonical 20 amino acids by the incorporation of non-canonical amino acids (ncaas) into proteins provides a mean to produce proteins with novel functions (Davis \& Chin, 2012). These ncaas include photocrosslinkers to study protein interactions (Chin et 
al, 2002; Liu et al, 2010), photocaged amino acids to activate a function in response to light (Lemke et al, 2007), post-translational modifications e.g. phosphoserine (Park et al, 2011), and spectroscopic probes (Ellis et al, 2008; Ye et al, 2010). Incorporation of fluorescent probes into proteins for intramolecular FRET measurements is of major interest in studies focusing on the investigation of conformational changes of proteins upon ligandbinding (Iijima \& Hohsaka, 2009; Kajihara et al, 2006; Khushoo et al, 2011), folding into the native conformation in solution (Borgia et al, 2011), upon interaction with chaperones (Sharma et al, 2008), or during co-translational protein folding on the ribosome (Woolhead et al, 2004). In many of those studies labeling was achieved by the co-translational incorporation of ncaas. In most cases a quantitative labeling of the model protein is of high importance to facilitate the interpretation of the fluorescence data. A future direction in this field might also be the investigation of translation rates and co-translational protein folding on the ribosome (Komar, 2009; Zhang et al, 2009). In this case additional requirements exist for the co-translational incorporation of ncaas like the incorporation rate, i.e. the incorporation of a ncaa should not slow down translation kinetics to any significant extent compared to a canonical amino acid. Non-canonical amino acids are incorporated during ribosomal protein synthesis by ncaa-tRNAs. Site specific incorporation is achieved by sense codon reassignment (using natural tRNAs carrying the non-canonical amino acid), suppression techniques such as nonsense suppression (Chin et al, 2003), or frameshift suppression by decoding of non-triplet coding units such as quadruplets using noncanonical tRNAs (Neumann et al, 2010; Rodriguez et al, 2006; Taki et al, 2002).

Protein biosynthesis has been evolutionarily optimized for the translation of canonical amino acids. The error frequency of protein synthesis ranges between $10^{-5}$ and $10^{-3}$ (Wohlgemuth et al, 2011). The same mechanisms that contribute to the fidelity of protein synthesis limit the incorporation of non-canonical amino acids. This includes the aminoacyl-tRNA synthetase, which acylates the amino acid to the corresponding tRNA, elongation factor $\mathrm{Tu}(\mathrm{EF}-\mathrm{Tu})$, which delivers the aminoacyl-tRNA (aa-tRNA) to the ribosome, and the ribosome which selects cognate aminoacyl-tRNAs based on the geometry of the codon-anticodon duplex and catalyzes peptide bond formation. To address the first step of the fidelity mechanism that ensures incorporation of correct canonical amino acids into proteins, aminoacyl-tRNA synthetase/tRNA pairs have been orthogonalized by in vivo selection to specifically aminoacylate their respective tRNAs with ncaas, like the leucyl-tRNA synthetase/tRNA ${ }^{\text {Leu }}$ pair (Wu et al, 2004), or the pyrrolysyl-tRNA synthetase/tRNA ${ }^{\text {Pyl }}$ pair (Hancock et al, 2010). EF-Tu in the GTP form 
binds to all cognate aa-tRNAs with similar high affinities (Louie \& Jurnak, 1985; Louie et al, 1984; Ott et al, 1989) which is essential for its function of tRNA delivery to the ribosome. However, experiments using misacylated tRNAs showed that EF-Tu binding to aminoacyl-tRNA is specific for the side chain of the esterified amino acid and that thermodynamic compensation is used to establish binding to all cognate aminoacyl-tRNAs with similar affinities (LaRiviere et al, 2001). In this context each aminoacyl-tRNA sequence seems to have evolved to adjust the affinity for binding to EF-Tu in a way that compensates for the affinity of its cognate amino acid (Dale \& Uhlenbeck, 2005a). In a similar way as the side chain of canonical amino acids can affect the acceptance of aminoacyl-tRNA by EF-Tu, artificial side chains of non-canonical amino acids can have a strong influence on the affinity of the ternary complex. An example of this is the lack of acceptance of (phosphoserine) Sep-tRNA ${ }^{\text {Sep }}$ by EF-Tu in E. coli (Park et al, 2011) or the low affinity of tRNA aminoacylated with bulky pyrenylalanine derivatives to EF-Tu (Doi et al, 2007). This problem has been addressed by introducing mutations in the amino acid binding pocket of EF-Tu, which aims at improving the binding of certain ncaa-tRNAs (Chapman et al, 2012; Doi et al, 2007; Park et al, 2011). Finally, synthetically evolved orthogonal ribosomes have been generated which show improved decoding of UAG stop codons (Wang et al., 2007) and quadruplet codons (Neumann et al, 2010) by certain ncaa$\mathrm{tRNA}^{\mathrm{aa}} \mathrm{s}$ in vivo.

Incorporation of ncaas has a multitude of applications in biological studies. One of the primary limitations of this technique still is the acceptance of ncaas by the translational apparatus. It has been reported that incorporation efficiencies of ncaas in many cases can be compromised depending on the nature of the site chain. In all of those studies the total incorporation efficiencies were quantified by the total amount of protein synthesized carrying ncaas compared to the amount synthesized using canonical amino acids (Doi et al, 2007; Hohsaka et al, 1999; Kajihara et al, 2006). This does not provide information about at which step of protein synthesis ncaas are disfavoured in comparison to canonical amino acids. Especially in the view of studies that must rely on an incorporation of a ncaa with speed and efficiency comparable to a canonical amino acid, e.g. investigation of cotranslational processes like protein folding, it would be desirable to understand the mechanisms that provide those limitations in kinetic detail and optimize the conditions that favor the incorporation of the ncaa. So far only one study has investigated the incorporation of ncaas by rapid kinetic analysis focusing on proline and other $\mathrm{N}$ alkylamino acids (Pavlov et al, 2009), establishing that the accommodation/peptidyl 
transfer step on the ribosome is rate limiting the incorporation for this particular class of ncaas.

Here we have chosen to analyze the incorporation of a fluorescent analog of lysine $\left(\varepsilon \mathrm{NH}_{2}\right.$-Bodipy576/589-Lys or BOP-Lys, Figure 1), which has already been shown to be useful in studies of protein folding (Woolhead et al, 2004), using rapid kinetic methods.

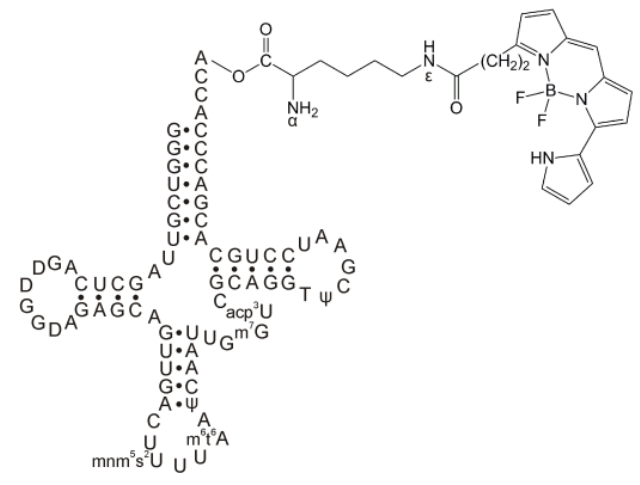

Figure 1 Cloverleaf structure of $\mathrm{tRNA}^{\mathrm{Lys}}$ from $E$. coli aminoacylated with the non-canonical $\varepsilon \mathrm{NH}_{2}-$ Bodipy576/589-Lysine, abbreviations for modified nucleotides are taken from (Sprinzl et al, 1998).

We identify steps during which the incorporation is compromised and optimize the conditions for an efficient incorporation, comparable to that of the canonical lysine. This study represents the first example of a pre-steady state kinetic analysis of the incorporation of a fluorescent ncaa during ribosomal protein synthesis. 


\section{Materials and Methods}

All chemicals were purchased from Merck, Sigma Aldrich, and Life Technologies. Isotope labeled amino acids were from Hartmann Analytic and Perkin Elmer. Messenger RNA (5'-GGCAAGGAGGUAAAUAAUGAAAUUCGUUAC-3' A site codon coding for Lys is underlined) was purchased from Microsynth.

\section{Buffers}

Buffer A Tris- $\mathrm{HCl}(50 \mathrm{mM}) \mathrm{pH} 7.5, \mathrm{NH}_{4} \mathrm{Cl}(70 \mathrm{mM}), \mathrm{KCl}(30 \mathrm{mM}), \mathrm{MgCl}_{2}(7 \mathrm{mM})$

Buffer B Hepes-KOH $(50 \mathrm{mM}) \mathrm{pH} 7.5, \mathrm{NH}_{4} \mathrm{Cl}(70 \mathrm{mM}), \mathrm{KCl}(30 \mathrm{mM}), \mathrm{MgCl}_{2}$ $(20 \mathrm{mM})$

Buffer C Tris- $\mathrm{CCl}(50 \mathrm{mM}) \mathrm{pH} 7.5, \mathrm{NH}_{4} \mathrm{Cl}(60 \mathrm{mM}), \mathrm{KCl}(30 \mathrm{mM}), \mathrm{MgCl}_{2}(7 \mathrm{mM})$, GDP $(50 \mu \mathrm{M})$, imidazole $(10 \mathrm{mM})$, glycerin $(15 \% \mathrm{v} / \mathrm{v}), \beta$-mercaptoethanol (5 mM), phenylmethylsulfonylfluorid $(0.1 \mathrm{mM})$

The $\mathrm{pH}$ of all buffers was adjusted at $20^{\circ} \mathrm{C}$, and the solutions were filtered through a $0.2 \mu \mathrm{m}$ cellulose acetate filter (Sartorius Stedim). $\beta$-mercaptoethanol and phenylmethylsulfonylfluorid were added immediately before use.

\section{Preparation of fMet-tRNA ${ }^{\text {fMet }}$ and $\alpha$ BodipyFL-Met-tRNA ${ }^{\text {fMet }}$}

Initiator fMet-tRNA ${ }^{\mathrm{fMet}}$ from E. coli was prepared as described (Milon et al, 2007). Met-tRNA ${ }^{\text {fMet }}$ was prepared in essentially the same way except that $\mathrm{N}^{10}$ formyltetrahydrofolate was omitted in the aminoacylation reaction. Met-tRNA ${ }^{\mathrm{fMet}}$ was modified using the aminoreactive sulfosuccinimidylester derivative of 4,4-difluoro-5,7dimethyl-4-bora-3a,4a-diaza-s-indacene-3-propionic acid (BodipyFL, Life Technologies, D6140) following the described protocol (Gite et al, 2000). After labeling, $\alpha$ BodipyFLMet-tRNA $^{\mathrm{fMet}}\left(\mathrm{BOF}-\mathrm{Met}_{\mathrm{TRNA}} \mathrm{fMet}^{\mathrm{fM}}\right.$ ) was ethanol precipitated at least 3 times to remove the excess of unreacted free dye. Traces of ethanol were removed by drying the tRNA pellet in a speedvac apparatus (Thermo Fisher). The dried tRNA pellet was dissolved in water and stored in aliquots at $-80^{\circ} \mathrm{C}$. The degree of fluorescence labeling was found to be complete as tested by hydrophobic interaction chromatography using isocratic elution in Buffer A on a Biosuite $250 \mathrm{HR}$ gel filtration column (Waters). 


\section{Preparation of $\mathrm{NH}_{2}$-Bodipy576/589-Lys-tRNA ${ }^{\mathrm{Lys}}$}

tRNA $^{\text {Lys }}$ was prepared from E. coli total tRNA (Roche) by purification on Sepharose 4B using reverse salt gradients and Phenyl-Sepharose (Cayama et al, 2000; Holmes et al, 1975). Aminoacylation of tRNA ${ }^{\text {Lys }}(30 \mu \mathrm{M})$ was carried out in buffer B supplemented with DTT $(2 \mathrm{mM})$, ATP $(3 \mathrm{mM}),\left[{ }^{14} \mathrm{C}\right]$ Lys $(40 \mu \mathrm{M})$ and $E$. coli lysyl-tRNA synthetase $(1 \% \mathrm{v} / \mathrm{v})$ (the plasmid coding for LysRS was kindly provided by R. Green, Johns Hopkins University) for $1 \mathrm{~h}$ at $37^{\circ} \mathrm{C}$. After the reaction was complete the reaction mix was phenolized (RNA-grade Phenol, Roth) and the tRNA, located in the water phase, precipitated with ethanol at $-20^{\circ} \mathrm{C}$. Aminoacyl-tRNA was centrifuged at $5000 \mathrm{~g}$ and $4{ }^{\circ} \mathrm{C}$ for $60 \mathrm{~min}$ and the pellet dissolved in water. Labeling of Lys-tRNA ${ }^{\text {Lys }}$ with the succinimidyl ester of 4,4-difluoro-5-(2-pyrrolyl)-4-bora-3a,4a-diaza-s-indacene-3propionic acid (Bodipy 576/589, Life Technologies D2225) was performed as described (Woolhead et al, 2004). Because labeling of Lys-tRNA ${ }^{\text {Lys }}$ with amine reactive dyes can yield a mixture of products, translationally active $\varepsilon \mathrm{NH}_{2}$-modified Lys-tRNA ${ }^{\text {Lys }}$ was separated from the $\alpha \mathrm{NH}_{2}$ - and $\alpha / \varepsilon \mathrm{NH}_{2}$-modified and unlabeled species using an ethanol gradient (5-40\%) on a reversed phase C-18 resin (LiChrospher, WP300, Merck).

\section{Preparation of translation factors and ribosomes}

70S ribosomes from E. coli MRE 600 and initiation factors were prepared as described (Milon et al, 2007; Rodnina \& Wintermeyer, 1995). Site-directed mutagenesis of tufB (EF$\mathrm{Tu}, \mathrm{E} 215 \mathrm{~A}$ and D216A) in the vector pET24a was carried out by quick change mutagenesis. DpnI digested DNA was transformed into E. coli Novablue cells (EMD) according to the manufacturer's protocol. SOC medium $(250 \mu \mathrm{l})$ was added, and the cells were grown for $30 \mathrm{~min}$ at $37^{\circ} \mathrm{C}$ without antibiotic and then streaked out on LB (Luria Bertani) agar plates supplemented with kanamycin $(30 \mu \mathrm{g} / \mathrm{ml})$ followed by incubation for $16 \mathrm{~h}$ at $37^{\circ} \mathrm{C}$. Single colonies were picked from the plates to inoculate $3 \mathrm{ml} \mathrm{LB}$ (Kan $30 \mu \mathrm{g} / \mathrm{ml}$ ) which was grown over night at $37^{\circ} \mathrm{C}$ in an orbital shaker (New Brunswick Innova 44) at $200 \mathrm{rpm}$. From these cultures plasmid DNA was prepared using the NucleoSpin Plasmid kit (Macherey Nagel). DNA was sequenced by Seqlab (Göttingen) and positive clones were identified by DNA alignment using the Lasergene Seqman software (DNASTAR). For expression, the plasmid DNA was transformed into E. coli BL(21)DE3 cells by heat shock treatment for $2 \mathrm{~min}$ at $42^{\circ} \mathrm{C}$, streaked out on LB agar plates (Kan $30 \mu \mathrm{g} / \mathrm{ml}$ ) and grown at $37^{\circ} \mathrm{C}$ for $16 \mathrm{~h}$. A single colony was picked from these plates and used to inoculate a 20-100 ml LB (Kan $30 \mu \mathrm{g} / \mathrm{ml}$ ) flask which was incubated over 
night at $37^{\circ} \mathrm{C}$ and vigorous shaking. On the next day, 6-8 LB (Kan $30 \mu \mathrm{g} / \mathrm{ml}$ ) flasks with $400 \mathrm{ml}$ medium each were inoculated from the preculture (inoculum size $1 \%(\mathrm{v} / \mathrm{v})$ ). These cultures were grown at $37^{\circ} \mathrm{C}$ until the $\mathrm{OD}_{595}$ reached 0.5 (after 3-4 h). The expression was induced by the addition of IPTG $(1 \mathrm{mM})$ and cells were grown for additional three hours, before they were harvested by centrifugation in a Beckman Avanti J-30I centrifuge at $10000 \mathrm{rpm},(\mathrm{JA}-10,10976 \mathrm{~g})$ at $4^{\circ} \mathrm{C}$ for $30 \mathrm{~min}$. Cell pellets were kept on ice, resuspended in buffer $\mathrm{C}$ using a homogenizer (Wheaton) and opened by Emulsiflex (Avestin) at $4{ }^{\circ} \mathrm{C}$. Cell lysates were centrifuged in a Beckman ultracentrifuge (Ti 50.2, $35000 \mathrm{rpm}$ $\left.(111338 \mathrm{~g}), 4^{\circ} \mathrm{C}\right)$ for $45 \mathrm{~min}$ and applied to a Protino column (6 ml, Macherey Nagel), operated in gravity flow mode. The resin was washed with 8 bed volumes buffer $\mathrm{C}$ before the protein was eluted in buffer $\mathrm{C}$ containing $250 \mathrm{mM}$ imidazole. The protein was subsequently concentrated in a Vivaspin 20 (30000 MWCO, Sartorius Stedim) and the buffer exchanged to buffer A using a diafiltration cup. The protein was concentrated to a final concentration of $300-500 \mu \mathrm{M}$, frozen in small aliquots in liquid nitrogen and stored at $-80^{\circ} \mathrm{C}$.

\section{Initiation and binary complex formation}

Initiation complexes were prepared in buffer A supplemented with DTT (2 mM) and GTP $(1 \mathrm{mM})$ by incubating $70 \mathrm{~S}$ ribosomes $(1 \mu \mathrm{M})$ in presence of IF 1,2 , and $3(1.5 \mu \mathrm{M}$ each), mRNA $(3 \mu \mathrm{M})$ and $\left[{ }^{3} \mathrm{H}\right] \mathrm{fMet}-\mathrm{RNA}{ }^{\mathrm{fMet}}(1.5 \mu \mathrm{M})$ or $\alpha \mathrm{NH}_{2}$-BodipyFL- $\left[{ }^{3} \mathrm{H}\right] \mathrm{Met}-$ $\operatorname{tRNA}^{\mathrm{fMet}}(1.5 \mu \mathrm{M})$ for $30 \mathrm{~min}$ at $37^{\circ} \mathrm{C}$. For purification, the initiation mix was pelleted through a sucrose cushion $(1.1 \mathrm{M})$ in buffer A. Centrifugation was performed in a TLS 55 swing-out rotor in a Beckman Optima XP ultracentrifuge at $4^{\circ} \mathrm{C}$ and $259000 \mathrm{~g}$ for $2 \mathrm{~h}$. After the centrifugation the supernatant was discarded and the ribosomal pellets were dissolved in buffer A on ice, frozen in small aliquots in liquid nitrogen and stored at $-80^{\circ} \mathrm{C}$. Initiation was monitored by nitrocellulose filtration and quantified as the amount of $\left[{ }^{3} \mathrm{H}\right]$ labeled initator tRNA retained on the filter (bound to ribosomes) by scintillation counting. Alternatively, if the fluorescent initiator tRNA and no purification was used, initiation was performed as described above except that $70 \mathrm{~S}$ ribosomes $(0.75 \mu \mathrm{M})$ were in 1.5 -fold excess over BOF- $\left[{ }^{3} \mathrm{H}\right] \mathrm{Met}-\mathrm{tRNA}{ }^{\mathrm{fMet}}(0.5 \mu \mathrm{M})$. Restriction of the concentration of initiator tRNA allowed to limit the amount of initiated ribosomes per mRNA $\leq 1$ and reduced the fluorescent background of the sample (originating from non-initiated BOF- $\left[{ }^{3} \mathrm{H}\right] \mathrm{Met}-$ tRNA $^{\text {fMet }}$ ). 
Binary complex of EF-Tu (wt and mutants) and GTP was formed by incubating EFTu.GDP $(5-25 \mu \mathrm{M})$ with GTP $(1 \mathrm{mM})$, DTT $(2 \mathrm{mM})$, phosphoenolpyruvate $(3 \mathrm{mM})$, and pyruvate kinase $(0.05 \mathrm{mg} / \mathrm{ml})$ in buffer $\mathrm{A}$ at $37^{\circ} \mathrm{C}$ for $15 \mathrm{~min}$ and was subsequently stored on ice until it was used for further experiments. Charging of binary complex with BodipyFL-GTP (G12411, Life Technologies) was carried out in essentially the same way, except that the reaction was done in the presence of $0.3 \mathrm{mM}$ BodipyFL-GTP and EF-Ts $(0.02 \mu \mathrm{M})$. To remove the excess of BodipyFL-GTP, the binary complex was purified using a NAP-5 column (Sephadex G-25, GE Healthcare).

\section{Kinetics of ternary complex formation, dissociation, and binding to the ribosome}

Ternary complex formation was measured in a SX-20MV stopped-flow apparatus (Applied Photophysics, Leatherhead, UK) monitoring the fluorescence changes upon rapid mixing of BOP-Lys-tRNA ${ }^{\text {Lys }}(0.05 \mu \mathrm{M})$ with variable amounts of EF-Tu.GTP in buffer A at $20^{\circ} \mathrm{C}$. Excitation was $560 \mathrm{~nm}$ and emission was monitored after passing a $590-\mathrm{nm}$ cutoff filter. Fluorescence changes were analyzed by fitting the experimental data to exponential functions in the form $\mathrm{F}=\mathrm{F}_{\infty}+\mathrm{A} \times \exp \left(-\mathrm{k}_{\mathrm{app}} \times \mathrm{t}\right) . \mathrm{K}_{\mathrm{d}}$ values were obtained by plotting the fluorescence amplitudes against the concentration of EF-Tu.GTP added to the reaction and fitting the data by a one-site binding model $\left(\mathrm{Y}=\mathrm{B}_{\max } \times \mathrm{X} /\left(\mathrm{K}_{\mathrm{d}}+\mathrm{X}\right)+\mathrm{NS} \times \mathrm{X}+\mathrm{F}\right)$, or in the case of the high affinity interaction (see results), by a quadratic equation $\left(\mathrm{Y}=\mathrm{M} \times\left(\left(\mathrm{A}+\mathrm{X}+\mathrm{K}_{\mathrm{d}}\right) / 2\right.\right.$ $\left.\left.\operatorname{SQRT}\left(\left(\mathrm{A}+\mathrm{X}+\mathrm{K}_{\mathrm{d}}\right)^{\wedge} 2 / 4-\mathrm{A} \times \mathrm{X}\right)\right)+\mathrm{F}\right)$. Association $\left(\mathrm{k}_{\mathrm{on}}\right)$ and dissociation rates $\left(\mathrm{k}_{\mathrm{off}}\right)$ were determined by plotting $\mathrm{k}_{\mathrm{app}}$ against the concentration of EF-Tu.GTP and analyzing the data by linear regression. Alternatively, dissociation rates were obtained by a chase experiment, in which the ternary complex EF-Tu.GTP $(2 \mu \mathrm{M})$ BOP-Lys-tRNA ${ }^{\mathrm{Lys}}(0.05 \mu \mathrm{M})$, was chased with an excess $(20 \mu \mathrm{M})$ of unlabeled total aa-tRNA ${ }^{\text {aa }}$. The $\mathrm{k}_{\text {off }}$ in these experiments was determined from the rate of the fluorescence decrease which was fitted by a single exponential function. Kinetics of the interaction of EF-Tu.GTP.BOP-Lys-tRNA ${ }^{\text {Lys }}$ and ribosomes with an AAA codon in the A site and $\mathrm{BOF}-\left[{ }^{3} \mathrm{H}\right] \mathrm{Met}-\mathrm{tRNA}{ }^{\mathrm{fMet}}$ in the $\mathrm{P}$ site, as well as the reaction of EF-Tu-BodipyFL-GTP-BOP-Lys-tRNA ${ }^{\text {Lys }}$ in presence of ribosomes with $\mathrm{f}\left[{ }^{3} \mathrm{H}\right] \mathrm{Met}^{-\mathrm{tRNA}}{ }^{\mathrm{fMet}}$ in the $\mathrm{P}$ site was measured by FRET (fluorescence resonance energy transfer) in the stopped-flow apparatus. The donor (BodipyFL) was excited at 470 $\mathrm{nm}$ and the fluorescence of the donor was monitored after passing a 500-nm cutoff filter, while the fluorescence of the acceptor (Bodipy576/589) was detected after passing through a 590-nm cutoff filter. 


\section{Kinetics of peptide bond formation by quench-flow analysis}

Equal volumes $\left(14 \mu \mathrm{l}\right.$ each) of ternary complex EF-Tu.GTP.BOP- $\left[{ }^{14} \mathrm{C}\right]$ Lys-tRNA ${ }^{\text {Lys }}$ $(0.1 \mu \mathrm{M})$ and initiated ribosomes $(0.3 \mu \mathrm{M})$ were rapidly mixed in a quench-flow apparatus (KIN-TEK Laboratories) and allowed to from peptide bonds before the reaction was quenched by potassium hydroxide $(0.5 \mathrm{M})$. Peptides were released by alkaline hydrolysis at $37^{\circ} \mathrm{C}$ for $30 \mathrm{~min}$. Subsequently, the $\mathrm{pH}$ was adjusted by addition of $1 / 10$ volume of acetic acid and all samples were filled up to the same volume by the addition of trifluoroacetic acid $(0.1 \%, \mathrm{v} / \mathrm{v})$. Dipeptides were analyzed by reversed-phase HPLC (LiChrospher WP300 RP-8, Merck) using a two-step linear gradient from 0 to $65 \%(\mathrm{v} / \mathrm{v})$ acetonitrile in $0.1 \%(\mathrm{v} / \mathrm{v})$ trifluoroacetic acid. The fractions $(1 \mathrm{ml})$ were collected, and after addition of Irga-Safe Plus (Perkin Elmer) scintillation cocktail $(2 \mathrm{ml})$ analyzed by liquid scintillation counting (Tri-Carb 3110 TR, Perkin Elmer). The fraction of dipeptide formed was calculated based on the ratio of fMetLys/(fMet+fMetLys) and analyzed by exponential fitting using one or two exponential terms. 


\section{Results}

\section{Stability of the ternary complex EF-Tu.GTP·aa-tRNA ${ }^{\text {a }}$ using a fluorescent derivative of Lys}

EF-Tu in complex with GTP binds to all canonical aminoacyl-tRNAs in a narrow range of high affinities forming a tight ternary complex. The kinetic parameters determining the stability of a ternary complex containing a non-canonical amino acid have so far not been determined in a quantitative manner. In order to determine the stability $\left(\mathrm{K}_{\mathrm{d}}\right)$ and the kinetic parameters for the association $\left(\mathrm{k}_{\mathrm{on}}\right)$ and dissociation $\left(\mathrm{k}_{\mathrm{off}}\right)$ of the ternary complex containing the fluorescent $\varepsilon \mathrm{NH}_{2}$-Bodipy576/589-Lys-tRNA ${ }^{\text {Lys }}$ (BOP-LystRNA $^{\text {Lys }}$ ) time courses of ternary complex formation were measured in a stopped-flow apparatus by mixing binary complex (EF-Tu.GTP) with BOP-Lys-tRNA ${ }^{\text {Lys }}$. Upon titration with increasing amounts of wild type EF-Tu.GTP the amplitude of the fluorescence signal increased substantially, along with a linear increase in the rate (Figure 2A).
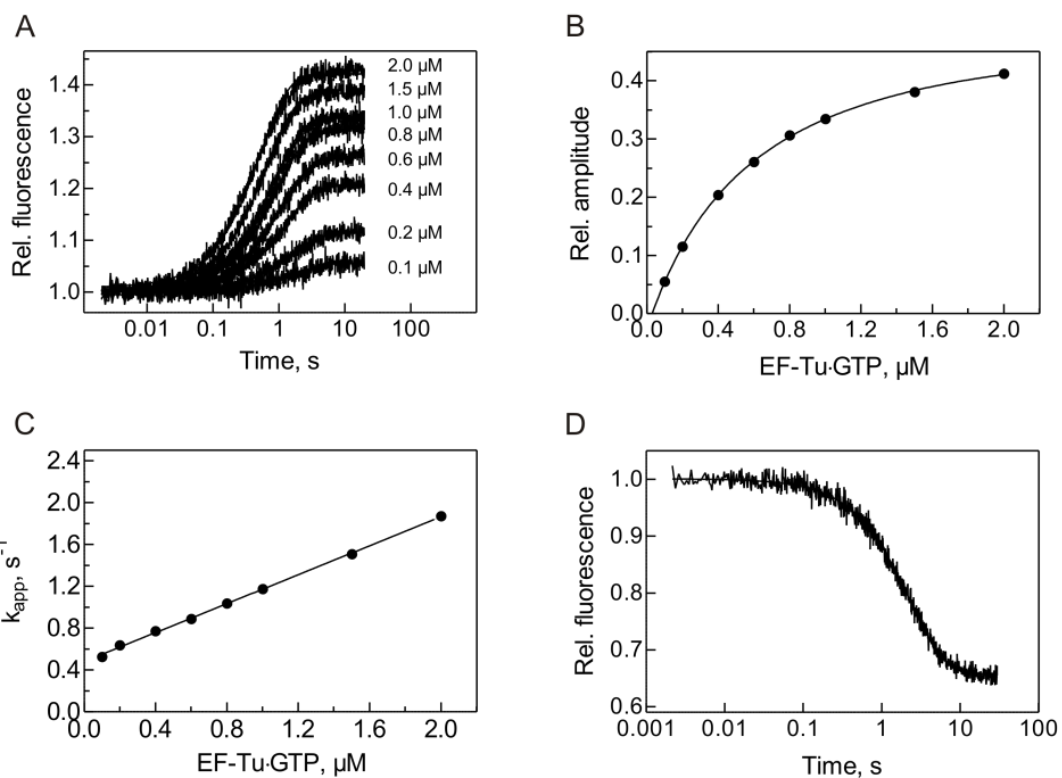

Figure 2 Ternary complex formation monitored by following fluorescence changes in the stopped-flow. (A) Time-resolved fluorescence change of BOP-Lys-tRNA ${ }^{\text {Lys }}$ forming the ternary complex EF-Tu.GTP.BOPLys-tRNA $^{\text {Lys }}$ at increasing concentrations of EF-Tu.GTP. Thin lines represent the fit of the fluorescent signal by single exponential functions. (B) Dependence of the relative fluorescence amplitude on the concentration of EF-Tu.GTP. The data was analyzed with a one-site binding model giving a $\mathrm{K}_{\mathrm{d}}=0.65 \pm 0.08 \mu \mathrm{M}$. (C) Dependence of $\mathrm{k}_{\mathrm{app}}$ on the concentration of EF-Tu.GTP. The data was analyzed by linear regression giving $\mathrm{k}_{\mathrm{on}}=0.69 \pm 0.01 \mu \mathrm{M}^{-1} \mathrm{~s}^{-1}$ (Slope) and $\mathrm{k}_{\text {off }}=0.48 \pm 0.01 \mathrm{~s}^{-1}$ (Y-Axis intercept). (D) Determination of the dissociation rate constant of the ternary complex by a chase experiment. Dissociation of the BOP-LystRNA $^{\text {Lys }}$ from the ternary complex was induced by mixing the ternary complex with a large excess of unlabeled aminoacyl-tRNA $(40 \mu \mathrm{M})$. The $\mathrm{k}_{\mathrm{off}}$, determined by single exponential fitting was $0.426 \pm 0.003 \mathrm{~s}^{-1}$.

The $K_{d}$ of the ternary complex was determined from evaluation of the fluorescence amplitudes, and found to be $0.65 \mu \mathrm{M}$ (Figure 2B, Table 1). The $\mathrm{k}_{\mathrm{app}}$ increased linearly with the increasing concentration of EF-Tu.GTP (Figure 2C), indicating that the observed 
fluorescence increase reflects binding. The $\mathrm{k}_{\text {on }}$ determined from this experiment was 0.69 $\mu \mathrm{M}^{-1} \mathrm{~s}^{-1}$ and the $\mathrm{k}_{\text {off }}$ was $0.48 \mathrm{~s}^{-1}$ which is similar to the $\mathrm{k}_{\text {off }}$ determined from an independent chase experiment $\left(0.43 \mathrm{~s}^{-1}\right.$, Figure $\left.2 \mathrm{D}\right)$ in which the ternary complex was rapidly mixed with a large excess (20-fold) of unlabeled aminoacyl-tRNA. This means that in comparison to the interaction of EF-Tu.GTP with a canonical aminoacyl-tRNA, the association of the non-canonical aa-tRNA with the factor is disfavored and the dissociation of the complex is facilitated, overall leading to a destabilization of the complex. The deficiency of binding of EF-Tu to bulky aromatic ncaa could arise from steric problems in the amino acid binding pocket of the factor. It was shown that mutations of two residues in the amino acid binding pocket of EF-Tu could recover the affinity for binding to bulky pyrenylalanine derivatives (Doi et al, 2007). To investigate the effect of the amino acids E215 and D216 in EF-Tu on the binding of BOP-Lys-tRNA ${ }^{\text {Lys }}$, the binding of BOP-Lys-tRNA ${ }^{\text {Lys }}$ to EF-Tu E215A, D216A, and E215A/D216A was tested using the fluorescence stopped-flow approach. The overall affinity of EF-Tu E215A to unmodified BOP-Lys-tRNA ${ }^{\text {Lys }}$ was 10-fold increased, which was mainly due to the decreased $k_{\text {off }}$ which was $0.04 \mathrm{~s}^{-1}$. The $\mathrm{k}_{\text {off }}$ determined for modified tRNA in a separate chase experiment shows a more than 35-fold decrease (Table 1).

Table 1 Binding of $\varepsilon \mathrm{NH}_{2}$-Bodipy576/589-Lys-tRNA ${ }^{\text {Lys }}$ to EF-Tu·GTP

\begin{tabular}{lllll}
\hline EF-Tu & $\mathrm{K}_{\mathrm{d}}, \mu \mathrm{M}$ & $\mathrm{k}_{\text {off }}, \mathrm{s}^{-1}$ & $\mathrm{k}_{\text {on }}, \mu \mathrm{M}^{-1} \mathrm{~s}^{-1}$ & $\mathrm{k}_{\text {off(chase })}, \mathrm{s}^{-1}$ \\
\hline wt & $0.65 \pm 0.08$ & $0.480 \pm 0.009$ & $0.692 \pm 0.008$ & $0.426 \pm 0.003$ \\
& $0.66 \pm 0.25^{\mathrm{a}}$ & $0.624 \pm 0.032^{\mathrm{a}}$ & $0.802 \pm 0.031^{\mathrm{a}}$ & \\
E215A & $0.06 \pm 0.04^{\mathrm{a}}$ & $0.045 \pm 0.008^{\mathrm{a}}$ & $0.454 \pm 0.007^{\mathrm{a}}$ & $0.0118 \pm 0.0001$ \\
D216A & $0.33 \pm 0.07^{\mathrm{a}}$ & $0.671 \pm 0.035^{\mathrm{a}}$ & $1.796 \pm 0.044^{\mathrm{a}}$ & $0.389 \pm 0.004$ \\
E215A/D216A & $0.013 \pm 0.007^{\mathrm{a}, \mathrm{b}}$ & $-0.048 \pm 0.017^{\mathrm{a}}(\sim 0)$ & $0.664 \pm 0.013^{\mathrm{a}}$ & $0.0035 \pm 0.0001$ \\
\hline
\end{tabular}

${ }^{\mathrm{a}}$ measured with transcribed tRNA

$\mathrm{b}$ evaluated using the square-root equation

For the mutant D216A the $\mathrm{k}_{\text {off }}$ was little affected, while the $\mathrm{k}_{\text {on }}$ was increased more than twofold leading to a slightly decreased $K_{d}$ (Table 1). Finally, the double mutant E215A/D216A had a substantially decreased $\mathrm{k}_{\text {off, }}$ as determined by chase experiments, which was about 120 -fold lower as the wild type. The $\mathrm{k}_{\mathrm{on}}$ instead was essentially the same as for wt EF-Tu which led to an overall $\mathrm{K}_{\mathrm{d}}$ in the range of $6-20 \mathrm{nM}$. Thus, both mutations E215A and D216A combined showed a greater increase for the affinity to BOP-LystRNA $^{\text {Lys }}$ in comparison to the single mutants, raising the affinity up to a level which is 
comparable to the stability of a ternary complex formed by wild type EF-Tu and a canonical tRNA.

\section{Translational activity of the ternary complex EF-Tu·GTP·BOP-Lys-tRNA ${ }^{\text {Lys }}$}

The translational activity of the ternary complex formed with BOP-Lys-tRNA ${ }^{\text {Lys }}$ was determined in a rapid dipeptide formation assay on ribosomes with a cognate lysine codon (AAA) in the A site. The ternary complex was formed during a short incubation (1 min) of BOP-Lys-tRNA $^{\text {Lys }}(0.2 \mu \mathrm{M})$ and a twofold excess of wild-type EF-Tu.GTP $(0.4 \mu \mathrm{M})$ before it was rapidly mixed with ribosomes in a quench-flow apparatus. The ternary complex with canonical Lys-tRNA ${ }^{\text {Lys }}$ formed dipeptides at a rate of $3.7 \mathrm{~s}^{-1}$ and had essentially completed the reaction after $1 \mathrm{~s}$. However, the reaction of the ternary complex with BOP-Lys-tRNA ${ }^{\text {Lys }}$ showed a biphasic behavior with an initial rapid rate of $2.7 \mathrm{~s}^{-1}$ and a slow rate of $0.07 \mathrm{~s}^{-1}$ not even reaching half completion after $1 \mathrm{~s}$ (Figure $3 \mathrm{~A}$ ). While the first rate is comparable to the dipeptide formation of the canonical ternary complex, the majority of the incorporation of the ncaa occurs at a rate which is not compatible with the average translation rate of most in vitro translation systems.
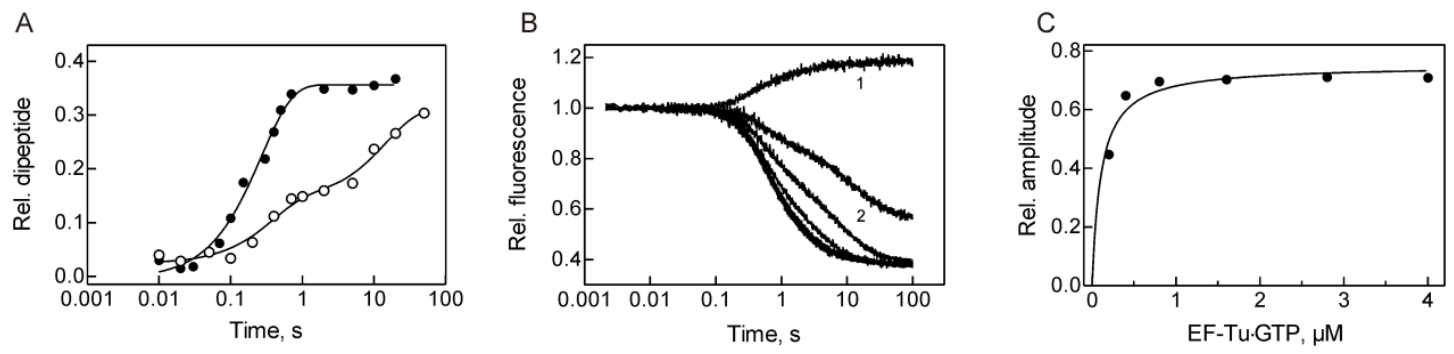

Figure 3 The low stability of the ternary complex including the ncaa impairs dipeptide formation on the ribosome. (A) Dipeptide formation of the ternary complex EF-Tu·GTP $(0.2 \mu \mathrm{M})$ BOP-Lys-tRNA ${ }^{\text {Lys }}(0.1 \mu \mathrm{M}$, open circles) and Lys-tRNA ${ }^{\text {Lys }}(0.1 \mu \mathrm{M}$, closed circles) upon binding to ribosomes with a cognate AAA codon in the A site $(0.3 \mu \mathrm{M})$. Thin lines represent fits by a single exponential term in the case of the wt LystRNA $^{\text {Lys }}$ giving a rate constant of $3.7 \pm 0.3 \mathrm{~s}^{-1}$ or a double exponential term in the case of the ternary complex containing BOP-Lys-tRNA ${ }^{\text {Lys }}$ giving rates of $2.7 \pm 0.9 \mathrm{~s}^{-1}$ and $0.07 \pm 0.02 \mathrm{~s}^{-1}$. (B) Binding of ternary complex $(0.1 \mu \mathrm{M})$ with Lys-tRNA ${ }^{\text {Lys }}(1)$ and BOP-Lys-tRNA ${ }^{\text {Lys }}$ (2) to ribosomes $(0.05 \mu \mathrm{M})$ with a cognate AAA codon in the A site and $\alpha$ BodipyFL-Met-tRNA ${ }^{\text {fMet }}$ in the P site. Shown is the donor (BodipyFL) fluorescence which is quenched in the presence of the acceptor fluorophore (2). The binding of ternary complex with BOP-Lys-tRNA ${ }^{\text {Lys }}$ was performed in presence of variable concentrations of EF-Tu.GTP (top to bottom: 0.2 $\mu \mathrm{M}, 0.4 \mu \mathrm{M}, 0.8 \mu \mathrm{M}, 1.6 \mu \mathrm{M}, 2.8 \mu \mathrm{M}$, and $4.0 \mu \mathrm{M}$ ), while for the binding of the ternary complex with LystRNA $^{\text {Lys }}$ only a concentration of $4.0 \mu \mathrm{M}$ is shown. (C) Dependence of the amplitude of donor fluorescence decrease on the concentration of EF-Tu.GTP. The maximum fluorescence amplitude should be obtained when half of the available BOP-Lys-tRNA ${ }^{\text {Lys }}$ is forming a ternary complex which binds to the ribosome, this is achieved at a concentration of $0.8 \mu \mathrm{M} \mathrm{EF-Tu} \cdot \mathrm{GTP}$.

In order to optimize the incorporation of BOP-Lys, the ternary complex was formed in the presence of different concentrations of EF-Tu.GTP to compensate for the low affinity of the factor for BOP-Lys-tRNA ${ }^{\text {Lys }}$. The binding to ribosomes was monitored in the 
stopped-flow by rapidly mixing the ternary complex with ribosomes with a fluorescent $\alpha$ BodipyFL-Met-tRNA $^{\text {fMet }}$ (BOF-Met-tRNA ${ }^{\text {fMet }}$ ) in the P site and a cognate lysine codon (AAA) in the A site. The binding of EF-Tu-GTP.BOP-Lys-tRNA ${ }^{\text {Lys }}$ to those ribosomes can be followed by FRET, which is visible in the acceptor induced quenching of the donor fluorescence (Figure 3B) and saturates at a concentration of $0.8 \mu \mathrm{M}$ representing $50 \%$ binding of all ternary complex available to ribosomes (Figure $3 \mathrm{C}$ ). Thus the binding of BOP-Lys-tRNA ${ }^{\text {Lys }}$ to the ribosome can be restored by addition of Tu, compensating for the low affinity of the factor to the tRNA.

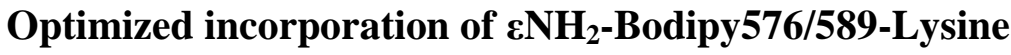

To determine the kinetics of ternary complex binding to the ribosome and the formation of the peptide bond in the absence of secondary effects resulting from the low affinity of EF-Tu for BOP-Lys-tRNA ${ }^{\text {Lys }}$, reactions were performed in the presence of an excess of EF-Tu.GTP $(4 \mu \mathrm{M})$, in order to achieve that more than $85 \%$ (based on $\mathrm{K}_{\mathrm{d}}=0.65$ $\mu \mathrm{M})$ of the available ncaa-tRNA was available as ternary complex. Peptide bond formation was measured by rapidly mixing EF-Tu.GTP.BOP-Lys-tRNA ${ }^{\text {Lys }}$ with an excess of ribosomes with an AAA codon in the A site and $\mathrm{f}\left[{ }^{3} \mathrm{H}\right] \mathrm{Met}_{-} \mathrm{RNA}{ }^{\mathrm{Met}}$ in the $\mathrm{P}$ site in a quench-flow apparatus. Using the ternary complex formed with EF-Tu (wild type), dipeptides formed at a rate of $1.7 \mathrm{~s}^{-1}$ (Figure 4A), which is comparable to the rate of peptide bond formation using the canonical amino acid. Furthermore, incorporation efficiency was comparable to that of the canonical Lys-tRNA ${ }^{\text {Lys }}$ (Figure 4A). The rate of peptide bond formation using the mutant EF-Tu (D216A) was very similar and had a rate of $1.6 \mathrm{~s}^{-1}$. However, the mutant EF-Tu (E215A) formed dipeptides at a rate of only $0.37 \mathrm{~s}^{-1}$ and the double mutant E215A/D216A was the slowest with a rate of only $0.07 \mathrm{~s}^{-1}$ (Figure 4A).

To investigate the processes determining the rate of peptide bond formation for the ncaa, the binding of the ternary complex carrying BOP-Lys-tRNA ${ }^{\text {Lys }}$ to the ribosome was tested using the FRET assay described above. The donor/acceptor pair used in these experiments (BodipyFL as donor, Bodipy576/589 as acceptor) had a calculated Förster radius $\left(\mathrm{R}_{0}\right)$ of $54 \AA$ assuming a random orientation of the fluorophores. In the $\mathrm{A} / \mathrm{T}$ state of decoding the aa-tRNA is still bound to EF-Tu and located approximately $90 \AA$ away from the peptidyltransferase center, where the donor on the $\alpha$-amino group of methionine is located (Figure 4B). At this distance the FRET efficiency should therefore be lower than 0.05. The accommodation of the acceptor-labeled tRNA in the A site, on the other hand, 
would bring the two fluorophores into very close proximity resulting in a FRET efficiency of close to 1, which in terms of the donor fluorescence would result in a significant decrease. Thus, a process leading to a major decrease of donor fluorescence is likely to reflect a state that follows after the tRNA has been released from EF-Tu (and is on its way towards the accommodated state), but is unlikely to represent a state in which the aatRNA $^{\text {aa }}$ is still bound to EF-Tu (in the A/T state).

A
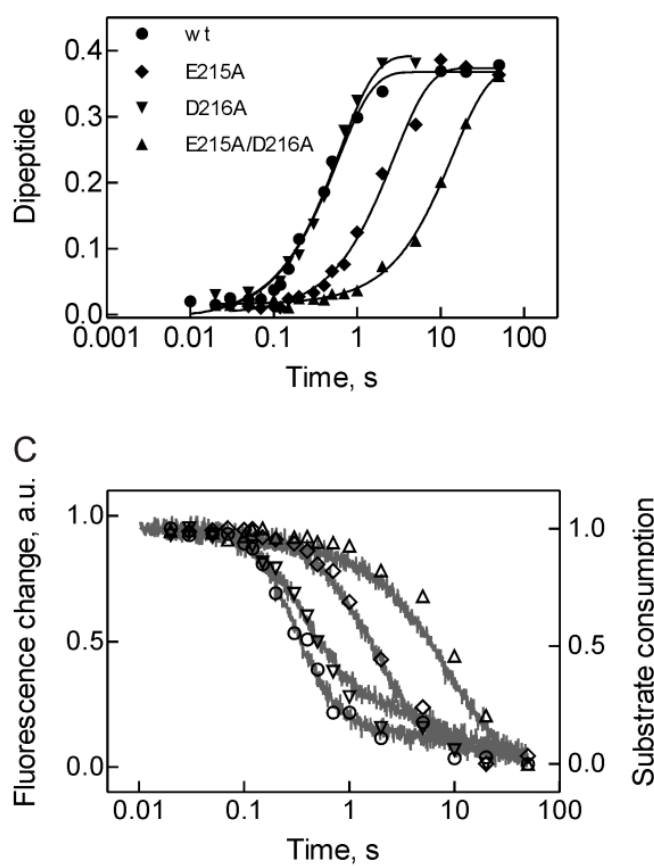

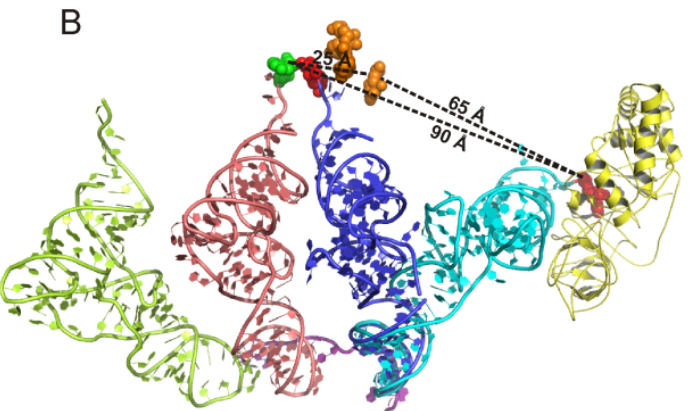

$\mathrm{D}$

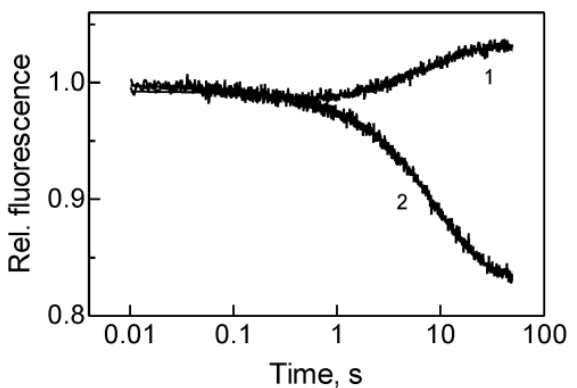

Figure 4 (A) Dipeptide formation of the ternary complex EF-Tu.GTP $(4 \mu \mathrm{M})$, BOP-Lys-tRNA ${ }^{\text {Lys }}(0.1 \mu \mathrm{M})$ upon binding to ribosomes with a cognate AAA codon in the A site $(0.3 \mu \mathrm{M})$. Thin lines represent fits by single exponential terms giving the rates of $1.74 \pm 0.13 \mathrm{~s}^{-1} \mathrm{EF}-\mathrm{Tu}$ wild-type (circles), $0.37 \pm 0.03 \mathrm{~s}^{-1} \mathrm{EF}-\mathrm{Tu}$ E215A (diamonds), $1.62 \pm 0.12 \mathrm{~s}^{-1}$ EF-Tu D216A (inverted triangles), and $0.071 \pm 0.004 \mathrm{~s}^{-1}$ EF-Tu E215A/D216A (triangles). (B) Structural rearrangement of the aa-tRNA upon accommodation in the A site of the ribosome. EF-Tu (yellow), aa-tRNA in the A/T state (cyan) and in the A/A state (dark blue), peptidyltRNA (salmon), E-site tRNA (light green) and mRNA (pink) are shown. The 3' terminal adenosines of the aa-tRNA and peptidyl-tRNA are highlighted representing the positions of the acceptor (red) and the donor (green) fluorophore, respectively. Bases C2556, U2492, and C2573 of 23S rRNA representing the accommodation gate are also highlighted (orange). The picture was generated from (Schmeing et al, 2009) (2WRN, 2WRO), except the aa-tRNA in the A/A state which was taken from (Yusupova et al, 2001) (1JGQ) and positioned by alignment of the $16 \mathrm{~S}$ rRNAs. (C) Acceptor induced quenching of the donor fluorescence upon binding of the ternary complex EF-Tu.GTP $(4 \mu \mathrm{M})$, BOP-Lys-tRNA ${ }^{\mathrm{Lys}}(0.1 \mu \mathrm{M})$ to ribosomes with a cognate AAA codon in the $\mathrm{A}$ site and $\alpha$ BodipyFL-Met-tRNA ${ }^{\text {fMet }}$ in the $\mathrm{P}$ site $(0.3 \mu \mathrm{M})$ and educt consumption upon peptide bond formation under the same concentrations. Left to right: EF-Tu wild-type (circles), EF-Tu D216A (inverted triangles), EF-Tu E215A (diamonds), EF-Tu E215A/D216A (triangles). The fluorescence decrease in the presence of BOP-Lys-tRNA ${ }^{\text {Lys }}$ occurs at a similar rate as educt consumption. (D) Release of BOP-Lys-tRNA ${ }^{\text {Lys }}(0.1 \mu \mathrm{M})$ from EF-Tu(E215A/D216A) BodipyFL-GTP $(4 \mu \mathrm{M})$ upon binding to ribosomes with a cognate AAA codon in the A site $(0.3 \mu \mathrm{M})$ as monitored by FRET (trace 1). The fluorescence signal of the donor was analyzed using a double exponential function giving a rate of $0.128 \pm 0.003 \mathrm{~s}^{-1}$ for the fluorescence increase. The rate of accommodation under the same conditions is only slightly slower $\left(0.114 \pm 0.001 \mathrm{~s}^{-1}\right.$, trace 2 , taken from Figure $\left.5 \mathrm{C}\right)$. 
Upon rapid mixing of EF-Tu(wt).GTP-BOP-Lys-tRNA ${ }^{\text {Lys }}$ with ribosomes with an AAA codon in the A site and an $\alpha$ BodipyFL-Met-tRNA ${ }^{\text {Met }}$ in the P site, using the same concentrations as in the peptide bond formation assay, the fluorescence signal of the donor showed a significant decrease (Figure 4C). To facilitate the comparison of the kinetics of peptide bond formation with the observed fluorescence changes in the FRET experiments, the consumption of ternary complex (substrate consumption) was plotted against the fluorescence change of the donor (Figure 4C). Since the rate of dipeptide formation is not influenced by the exchange of the formyl-group on the $\alpha$-amino group of methionine by BodipyFL (personal communication by Ingo Wohlgemuth and Wolf Holtkamp) it could be excluded that the presence of the donor fluorophor had any (accelerating or decelerating) influence on the reaction. Notably, the fluorescence trace describes the data points obtained for ternary complex consumption quite precisely, which means that peptide bond formation and acceptor induced quenching of the donor fluorescence (movement of the tRNA into the accommodated state) occur concomitantly. If the same experiment was performed with the ternary complex formed with EF-Tu (D216A) the fluorescence decrease showed a comparable amplitude but was slightly slower. In the case of the E215A mutant the rate of the fluorescence decrease was even slower and for the E215A/D216A mutant it was found to be the slowest (>30-fold slower than the wild type). In all cases the kinetics of the fluorescence decrease were described very well by the kinetics of substrate consumption. This indicated that the movement of the tRNA into the accommodated state (or any earlier step such as release from EF-Tu) but not the chemistry of peptide-bond formation was the rate-limiting step for the incorporation of BOP-Lysine. Rather, as the acceptor labeled tRNA approached the donor closer than $25 \AA$ the maximal FRET efficiency was reached (E>0.99) and peptide bond formation followed instantly (Figure 4C). Thus, the movement of the fluorescently labeled amino acid was not significantly paused at the accommodation gate formed by residues C2556, U2492, and C2573 of 23S rRNA (Figure 4B). Since these residues are located only 20-25 $\AA$ away from the peptiyltransferase center a pause in the tRNA movement would be reflected in the FRET signal, i.e. it should saturate faster than peptide bond formation takes place.

To address the earlier steps of BOP-Lysine incorporation, preceding accommodation and peptide bond formation, we analyzed the release of BOP-Lys-tRNA ${ }^{\text {Lys }}$ from EF-Tu, measuring FRET between EF-Tu-BodipyFL-GTP and BOP-Lys-tRNA ${ }^{\text {Lys }}$. In the ternary complex BodipyFL-GTP and BOP-Lysine on the tRNA are located in close proximity (approximately $25 \AA$ apart) which is expected to result in an efficient FRET signal 
$(\mathrm{E} \approx 0.99)$. Upon release of the tRNA from the factor on the ribosome this FRET signal should be significantly decreased. We determined the rate of tRNA release from EF$\mathrm{Tu}(\mathrm{E} 215 \mathrm{~A} / \mathrm{D} 216)$, which in the previous experiments exhibited the slowest kinetics of accommodation and peptide bond formation. The rapid mixing of EF$\mathrm{Tu}(\mathrm{E} 215 \mathrm{~A} / \mathrm{D} 216 \mathrm{~A}) \cdot$ BodipyFL-GTP.BOP-Lys-tRNA $^{\text {Lys }}$ with ribosomes resulted in a fluorescence increase of the donor (release of fluorescence quenching), at a rate of $0.13 \mathrm{~s}^{-1}$ (Figure 4D, trace 1). This rate is very similar to the rate of accommodation (Figure 4D, trace 2, $0.11 \mathrm{~s}^{-1}$ ) measured at the same concentrations of ribosomes, EF-Tu and BOP-LystRNA $^{\text {Lys }}$. Finally, this indicates that the incorporation of BOP-Lysine is limited by the release of the aa-tRNA from EF-Tu(E215A/D216A), while the later steps like accommodation and peptide bond formation can occur at rates which are comparable to the incorporation of the canonical amino acid (Figure 4C).

\section{Discussion}

Here we present pre-steady state experiments for the incorporation of a non-canonical amino acid on the $E$. coli ribosome using a fluorescent derivative of Lys-tRNA ${ }^{\text {Lys }}$ labeled with Bodipy576/589 at the $\varepsilon$-amino group of lysine. The results might help to rationalize the experimental observations of low incorporation efficiencies reported in many studies which make use of non-canonical amino acids for protein modification (Doi et al, 2007; Kajihara et al, 2006) and help to identify conditions for an efficient incorporation of ncaas. By analysis of the stability of the ternary complex EF-Tu.GTP.BOP-Lys-tRNA ${ }^{\text {Lys }}$ we find that the affinity is extremely low $\left(\mathrm{K}_{\mathrm{d}}=0.65 \mu \mathrm{M}\right)$ and about 100-fold lower than that of a canonical ternary complex. Resulting from a marked increase in the $k_{\text {off }}$ value $\left(0.4 \mathrm{~s}^{-1}\right)$ for aa-tRNA dissociation from the complex. Furthermore, the overall stability of the ternary complex including the non-canonical amino acid seems not to be significantly influenced by the presence of modifications on the tRNA body itself (Table 1). This could be due to the overall lower affinity caused by the ncaa masking the subtle differences caused by the base modifications of the tRNA. However, it might also be explained by the fact that only one of the modified bases in E. coli Lys-tRNA $^{\text {Lys }}$, the pseudouridine at position 54, belongs to the group of residues that are in close contact to EF-Tu in the ternary complex (Nissen et al, 1995; Sanderson \& Uhlenbeck, 2007). Furthermore, it has been shown that tRNA ${ }^{\text {Phe }}$ from $E$. coli can adopt the same overall fold irrespective of the presence or absence of base modifications (Byrne et al, 2010). 
The amino acid binding pocket of EF-Tu is located at the interface between domains 1 and 2. In several cases site-directed mutagenesis of residues in the amino acid binding pocket of EF-Tu has been reported to result in an altered specificity of EF-Tu for aa-tRNA binding (Chapman et al, 2012; Dale et al, 2004). Alternatively, EF-Tu was orthogonalized using a random mutagenesis approach, to form a functional ternary complex with a particular aa-tRNA substrate, which is not accepted by wild type EF-Tu (Park et al, 2011). Exchange of the residues E215 or D216 to alanines improved the incorporation of L-2anthraquinonylalanine, L-2-pyrenylalanine and L-1-pyrenylalanine into proteins (Doi et al, 2007), which might simply be attributed to an increase in the size of the amino acid binding pocket, minimizing the possibility of a steric clash between bulky substrates and those residues.

The mutation of residues E215 and D216 in EF-Tu to alanines also increases the affinity for BOP-Lys-tRNA ${ }^{\text {Lys }}$. The most prominent effect, as seen for the double mutant, is a 50-fold increase in the affinity, which is mainly resulting from a decrease in the $\mathrm{k}_{\text {off }}$ rate. Similar parameters characterize the interaction of the E215A mutant with the ncaatRNA, although the $K_{d}$ is only lowered by a factor of 10 . For the D216A mutant in contrast, the 2-fold lowered $\mathrm{K}_{\mathrm{d}}$ can be mainly attributed to an increased $\mathrm{k}_{\mathrm{on}}$, thus for this mutant the rate of binding is even increased, while the $\mathrm{k}_{\text {off }}$ is similar to that of the wild type protein. The low affinity of the ternary complex with the ncaa and wild type EF-Tu helps to explain the slow rate of peptide bond formation on the ribosome (Figure 4A), since only a small part of non-canonical aminoacyl-tRNA is constantly bound to EF-Tu, and only this part is active in rapid peptide bond formation. The majority of the ncaa-tRNA instead is only incorporated during a second, slow round of peptide bond formation $\left(0.07 \mathrm{~s}^{-1}\right)$, which is likely to be determined by the rate of aa-tRNA binding to EF-Tu.GTP. Given the low affinity of the ternary complex it seems feasible to speculate that the incorporation of this amino acid will be low in translation systems in which there is no significant excess of EF$\mathrm{Tu} \cdot \mathrm{GTP}$ over all available aminoacyl-tRNAs. Also compensation by addition of an excess of ncaa-tRNA is unlikely to improve efficiency, since the canonical aa-tRNA can easily outcompete the ncaa-tRNA for ternary complex formation if EF-Tu is limiting. As expected the addition of an excess of EF-Tu-GTP leads to an increased binding of ncaatRNA to the ribosome (Figure 4BC). Furthermore, kinetics of peptide bond formation become rapid, with $1.7 \mathrm{~s}^{-1}$ still about 2 -fold slower than the wild type tRNA at comparable conditions $\left(3.7 \mathrm{~s}^{-1}\right)$. Improving the ternary complex stability by the use of more tightly binding EF-Tu mutants in turn does not improve the rate of peptide bond formation on the 
ribosome in a straightforward manner. Instead we find a positive correlation between the $\mathrm{k}_{\text {off }}$ rate for ternary complex dissociation and the rate of dipeptide formation on the ribosome (Figure 4A, Table 1). For example the ternary complex EF$\mathrm{Tu}(\mathrm{E} 215 \mathrm{~A} / \mathrm{D} 216 \mathrm{~A}) \cdot \mathrm{GTP} \cdot \mathrm{BOP}-\mathrm{Lys}-\mathrm{tRNA}{ }^{\text {Lys }}$ is forming the tightest complex and has the lowest $\mathrm{k}_{\text {off }}$ of $0.0035 \mathrm{~s}^{-1}$, but also has the slowest rate of peptide bond formation with a rate of $0.07 \mathrm{~s}^{-1}$ (this extremely slow rate of dipeptide formation might help to explain, why this mutant shows no translational activity in the assay described by Doi et al., 2007). On the other hand, the ternary complex with EF-Tu(D216A) is 2-fold more stable than the wild type. This is mainly due to an increased $\mathrm{k}_{\text {on }}$, however it has a very similar $\mathrm{k}_{\text {off }}$ compared to wild type EF-Tu, and is forming dipeptides with almost the same rate as wild type EF-Tu. This result seems to be in line with the results reported in a study where a strong correlation between the $\mathrm{k}_{\text {off }}$ of the aa-tRNA dissociation from the ternary complex, formed by EF-Tu-GTP and various aa-tRNAs, including mutants and misacylated tRNAs, and the rate of peptide bond formation on the ribosome was described (Schrader et al, 2011).

If the slow $\mathrm{k}_{\text {off }}$ for ncaa-tRNA dissociation from EF-Tu-GTP(E215A/D216A) indeed is related to the slow release of the tRNA from EF-Tu.GDP on the ribosome remains to be established. However, since canonical ternary complexes also show a comparably low $\mathrm{k}_{\text {off }}$ for ternary complex dissociation (Louie \& Jurnak, 1985; Louie et al, 1984; Ott et al, 1989), but exhibit rapid peptide bond formation on the ribosome (Wohlgemuth et al, 2010), it seems likely that other factors also play a role like correct binding of the EF-Tu mutant to the ribosome, e.g. formation of an important interaction of histidine 84 with A2662 of the sarcin-ricin loop (Voorhees et al, 2010) which might influence the rate of GTPase activation upon correct codon-anticodon interaction.

Using FRET-based assays we were able to follow the release of the fluorescent ncaa$\mathrm{tRNA}^{\text {aa }}$ from EF-Tu and its accommodation into the A-site of the ribosome. Indeed we find that for the mutant E215A/D216A the release of ncaa-tRNA $\left(0.13 \mathrm{~s}^{-1}\right)$ could be ratelimiting all the further steps including accommodation and peptide bond formation (Figure 4D). Accommodation, as monitored by the FRET change between the labeled amino acids of the intitiator tRNA ${ }^{\text {Met }}$ and the elongator Lys-tRNA ${ }^{\text {Lys }}$, proceeds at the same rate as peptide bond formation supporting the view that also tRNAs with highly modified amino acids are able to pass the accommodation gate without significant delay (Sanbonmatsu et al, 2005). Furthermore, the rate of peptide bond formation is not delayed compared to the rate of accommodation, meaning that the modification of Lys does not affect the chemistry of peptide bond formation to a significant extent. This is in line with the fact that the 
ribosome is able to accept a wide range of structurally diverse substrates for efficient catalysis, mainly by lowering the entrophy of activation (Sievers et al, 2004).

On the ribosome cognate aa-tRNAs are discriminated at three different selection stages (Wohlgemuth et al, 2011). During initial selection the formation of a cognate codonanticodon duplex triggers conformational changes in the decoding complex, which lead to rapid GTP hydrolysis in EF-Tu. In the GDP form EF-Tu has a low affinity for the aa-tRNA and dissociates from the ribosome. In the second proofreading step the ribosome controls the differences in the stability of the codon-anticodon complex and selectively accelerates the rate of accommodation for cognate substrates while for near-cognate substrates dissociation is favored. It has also been shown that erroneous peptidyl-tRNAs can be preferentially hydrolyzed by termination factors (Zaher \& Green, 2009b). While during all steps described above the discrimination of codon-anticodon duplex in the A or P site seems to be most critical, some evidence may suggest that the ribosome is also able to show some discrimination against misaminoacylated tRNAs which are cognate for the mRNA codon in the A site, if the binding is sufficiently weak (Dale et al, 2009). In this study, a fully modified cognate tRNA ${ }^{\text {Lys }}$ body was used. While we did not analyze the hydrolysis of the nascent peptide containing the fluorescently modified lysine in the presence of release factors, from the rates of peptide bond formation using BOP-LystRNA $^{\text {Lys }}$ and Lys-tRNA ${ }^{\text {Lys }}$, we can conclude that the ribosome may have exerted only a mild discriminatory effect during initial selection and proofreading against the noncanonical tRNA. However, given the excess of EF-Tu that was used, we might not be able to exclude a rebinding of an already rejected aa-tRNA to EF-Tu.GTP, which then would have the possibility to enter a new round of selection.

EF-Tu has evolved to bind canonical correctly aminoacylated tRNA with uniform high affinities. One function is clearly the protection of aa-tRNAs from deacylation in the cellular milieu under physiological $\mathrm{pH}$ but furthermore it also contributes as an active part of the quality control mechanism of translation to the selection of correctly aminoacylated tRNAs (LaRiviere et al, 2001). While the thermodynamic contributions of the amino acid and the tRNA body for the binding to EF-Tu are balanced in a canonical aa-tRNA, they can be imbalanced in a misacylated tRNA, leading to significantly weaker or stronger binding. In this context, the non-canonical aa-tRNA studied here might be compared to misacylated tRNAs, since no correct tRNA body exists for this ncaa. The improvement of ternary complex stability by the use of more tightly binding mutants of EF-Tu seems to be a valuable tool for the generation of labeled proteins in translation systems in which a large 
excess of EF-Tu over tRNA cannot be used and in which strong effects of translation rate on protein folding are not expected. However, they may not be suitable for pre-steady state incorporation of fluorophores in studies which are aiming at an investigation of the fine tuning of translation speed and protein folding, since they can slow down the translation rate. Taken together, this supports the view that the ribosome is able to accept tRNAs even with highly modified amino acids as substrates, while the low affinity of binding to EF-Tu imposes the greatest discriminatory effect on the selection against non-canonical aatRNAs. 


\title{
CHAPTER III: ANALYSIS OF NON-UNIFORM ELONGATION KINETICS DURING mRNA TRANSLATION BY SINGLE RIBOSOMES
}

\begin{abstract}
Local rates of protein chain elongation are variable. This non-uniformity of translation has functional importance for many cellular processes such as metabolic regulation of gene expression, membrane targeting of proteins, protein export and folding. The regulation of translation elongation rates on natural mRNAs is highly complex and dependent on many factors like codon usage, ribosome traffic, cooperation of ribosomes with other complexes, and initiation and termination rates, which has made it difficult to study using in vitro kinetic approaches. Here we have analyzed a single round of translation elongation on a natural full-length mRNA using one ribosome per mRNA molecule. Translation is paused at distinct sites, many of them but not all seem to be associated with rare codon clusters. Analysis of the formation and decay kinetics of each intermediate suggests that translation elongation follows rather a stochastic than a sequential manner. The stochastic movement of ribosomes observed in our system indicates that translation elongation represents one of the sources of gene-intrinsic noise in protein expression.
\end{abstract}

\section{Introduction}

The ribosome synthesizes proteins from aminoacyl-tRNAs (aa-tRNAs) based on the sequence of codons of an mRNA template. During each elongation step, aa-tRNA is delivered to the ribosome in a complex with elongation factor Tu (EF-Tu) and GTP. Following GTP hydrolysis by EF-Tu, the aa-tRNA is released from the factor, accommodates in the A site of the ribosome, and takes part in peptide bond formation. Then, elongation factor $\mathrm{G}(\mathrm{EF}-\mathrm{G})$ translocates the peptidyl-tRNA from the A site to the $\mathrm{P}$ site of the ribosome, and dissociates leaving an empty A site ready for the next round of elongation. Protein synthesis is characterized by the sequential repetition of the elongation cycle. Rather than being a uniform process, local rates of protein chain elongation can be variable and result in translational pausing at some regions of an mRNA molecule (Buchan $\&$ Stansfield, 2007). There are numerous examples of non-uniform translation rates, such as the irregular distribution of nascent protein chains (Krasheninnikov et al, 1991; Protzel \& Morris, 1974; Varenne et al, 1984) and ribosomes (Wolin \& Walter, 1988) during translation of particular mRNAs in vitro. Non-uniform translation rates in vivo are evident from differences in ribosome density along translated mRNAs as revealed by analysis of 
ribosome protected mRNA fragments after nuclease digestion (Ingolia et al, 2009; Kim \& Hollingsworth, 1992; Li et al, 2012).

The rate of translation elongation can be influenced by many different factors. If more than one ribosome translates a single mRNA at a time, this can result in the formation of a polysome, in which ribosomes can collide with each other and mutually influence their translation speed (Mitarai et al, 2008). The rate of translation can also be affected by cooperation of the translating ribosome with an RNA polymerase (Burmann et al, 2010; Proshkin et al, 2010). Furthermore, the unfolding of mRNA secondary structures can represent a kinetic barrier causing a decrease in elongation rates (Qu et al, 2011; Wen et al, 2008). Translation can also be arrested when the ribosome translates a stalling signal (Ito et al, 2010). The interaction between the anti-Shine-Dalgarno sequence of the 16S rRNA and internal Shine-Dalgarno-like sequences in the mRNA has also been suggested to cause translational pausing ( $\mathrm{Li}$ et al, 2012). Moreover, the electrostatic potential of the ribosomal tunnel can have an influence on translation elongation rates due to specific interactions between the tunnel which is negatively charged and the nascent peptide, which may contain positively charged arginine or lysine residues and can induce transient arrest of translation elongation ( $\mathrm{Lu} \&$ Deutsch, 2008). Non-uniformity of translation elongation rates might also be an intrinsic property of each individual translation elongation step. The rates governing the selection of individual cognate tRNAs on the ribosome are intrinsically uniform (Ledoux \& Uhlenbeck, 2008) with proline, which accommodates slower than other canonical amino acids, being an exception (Pavlov et al, 2009). Furthermore, the presence of a Watson-Crick base pair instead of a wobble pair in the third position of the codon anticodon-duplex has been shown to result in an increased rate of GTP hydrolysis in EF-Tu (Gromadski et al, 2006; Thomas et al, 1988). The abundance of individual tRNAs in the cell varies considerably (Dong et al, 1996; Ikemura, 1985) and the spatial movement of an aa-tRNA through the cytoplasm is limited by diffusion, meaning that the average arrival time of a particular tRNA at the ribosomal A site is inversely proportional to its concentration in the cell (Fluitt et al, 2007). Accordingly, codon-usage bias, which has no influence on the primary sequence of the protein synthesized (Plotkin \& Kudla, 2011), might have an influence on translation elongation rates (Komar, 2007). For example, there are six codons coding for arginine. In E. coli these codons are read by four different isoacceptor tRNAs, representing a fraction of $0.7 \%-7.4 \%$ of the total tRNA pool available in the cell (Dong et al, 1996). Aa-tRNA availability is further influenced by the recharging rates of deacylated tRNAs by aminoacyl-tRNA synthetases, which in turn can 
be limited by the availability of amino acids (Elf et al, 2003), e.g. under conditions of amino acid starvation (Dittmar et al, 2005).

Non-uniform translation elongation rates have a central role in many cellular processes ranging from metabolic regulation of gene expression, e.g. translational pausing at tryptophan codons is used to regulate the transcription of genes coding for proteins involved in tryptophan amino acid biosynthesis (Yanofsky, 2004), monitoring of the protein export activity of the cell (Nakatogawa et al, 2005), and SRP-mediated targeting of nascent membrane proteins (Walter \& Blobel, 1981). Furthermore, modulation of the rates of translation elongation might also be important for protein folding (Jha \& Komar, 2011; Komar, 2009), which is suggested by experiments showing that synonymous codon substitutions can have effects on the function and stability of the protein synthesized (Cortazzo et al, 2002; Kimchi-Sarfaty et al, 2007; Komar et al, 1999; Zhang et al, 2009).

Despite the central role of the regulation of translation elongation rates, there is a substantial lack of understanding how ribosomes actually move along the coding regions of natural full length mRNA molecules. A number of models have been developed to predict local translation elongation rates (Clarke \& Clark, 2008; Fluitt et al, 2007; Zhang \& Ignatova, 2009; Zouridis \& Hatzimanikatis, 2008). However, kinetic data is scarce and information on the local rates of translation elongation on long natural mRNAs is essentially missing. Methods capable of identifying sites of translational pausing (Ingolia et al, 2009; Varenne et al, 1984; Wolin \& Walter, 1988) thus far could only provide snapshots of ribosome distributions along an mRNA molecule or nascent protein chains synthesized under conditions of multiple-turnover and with limited time resolution. Recently, a method was introduced which allowed to study synchronized translation elongation of ribosomes resuming translation of a long mRNA molecule after being stalled at an internal isoleucine codon (Endoh et al, 2012). Here we have studied translation elongation kinetics for a full-length natural mRNA coding for the E. coli protein methyltransferase PrmC in a completely reconstituted E. coli translation system using purified ribosomes, elongation factors, and aa-tRNAs. This approach allowed us to follow translation elongation kinetics on synchronized ribosomes using one ribosome per mRNA. 


\section{Materials and Methods}

All chemicals were purchased from Merck, Sigma Aldrich, and Life Technologies. $\left[{ }^{3} \mathrm{H}\right]$-labeled methionine was from Hartmann Analytic.

\section{Buffers}

Buffer A Tris- $\mathrm{HCl}(50 \mathrm{mM}) \mathrm{pH} 7.5, \mathrm{NH}_{4} \mathrm{Cl}(70 \mathrm{mM}), \mathrm{KCl}(30 \mathrm{mM}), \mathrm{MgCl}_{2}(7 \mathrm{mM})$

Buffer B Tris- $\mathrm{HCl}(50 \mathrm{mM}) \mathrm{pH} 7.5, \mathrm{NH}_{4} \mathrm{Cl}(70 \mathrm{mM}), \mathrm{KCl}(30 \mathrm{mM}), \mathrm{MgCl}_{2}$ (3.5 mM), spermidine $(0.5 \mathrm{mM})$, putrescine $(8 \mathrm{mM})$, DTT $(2 \mathrm{mM})$

Buffer C Tris- $\mathrm{CCl}(50 \mathrm{mM}) \mathrm{pH} 7.5, \mathrm{NH}_{4} \mathrm{Cl}(70 \mathrm{mM}), \mathrm{KCl}(30 \mathrm{mM})$, spermidine $(1 \mathrm{mM})$, putrescine $(16 \mathrm{mM})$, DTT $(4 \mathrm{mM})$

Buffer D Hepes-KOH pH $7.5(50 \mathrm{mM}), \mathrm{NH}_{4} \mathrm{Cl}(70 \mathrm{mM}), \mathrm{KCl}(30 \mathrm{mM}), \mathrm{MgCl}_{2}$ $(20 \mathrm{mM})$

Buffer E $\quad \mathrm{NaOAc} p H 4.5(50 \mathrm{mM}), \mathrm{MgCl}_{2}(10 \mathrm{mM})$

Buffer F Tris- $\mathrm{FCl}$ pH $7.5(40 \mathrm{mM}), \mathrm{MgCl}_{2}(15 \mathrm{mM})$, spermidine $(2 \mathrm{mM}), \mathrm{NaCl}$ $(10 \mathrm{mM})$

After $\mathrm{pH}$ adjustment all solutions were filtered through a $0.2 \mu \mathrm{m}$ cellulose acetate filter (Sartorius Stedim).

\section{Preparation of translation factors and ribosomes}

Ribosomes from E. coli MRE 600, initiation factors, and EF-G were prepared as described (Milon et al, 2007; Rodnina et al, 1999; Rodnina \& Wintermeyer, 1995). EF-Tu containing a C-terminal oligohistidine tag was expressed and purified according to an established protocol (Wieden et al, 2002).

\section{Preparation of aa-tRNA}

BodipyFL-Met-tRNA ${ }^{\text {fMet }}$ (BOF-Met-tRNA ${ }^{\text {fMet }}$ ) was prepared according to the protocol detailed elsewhere (Chapter II). To prepare the mixture of aa-tRNA for translation, total tRNA from E. coli MRE 600 (Roche) was dissolved in potassium acetate (pH 5, $0.2 \mathrm{M}$ ) and extracted twice with an equal volume of phenol ( $\mathrm{pH} 4.5$, RNA grade, Roth). The tRNA was ethanol precipitated 4 times to remove traces of phenol. After the final centrifugation step at $5000 \mathrm{~g}$ for $1 \mathrm{~h}$ at $4^{\circ} \mathrm{C}$, the tRNA pellet was dried in a speedvac apparatus 
(Thermo Fisher) and dissolved in RNase-free water. Aminoacylation of total tRNA (80 unit $\left.\mathrm{A}_{260} / \mathrm{ml}\right)$ was performed in buffer D supplemented with ATP (3 mM), DTT (2 mM), and L-amino acids (Sigma, $300 \mu \mathrm{M}$ each), using S100 (3\% (v/v)) for $1 \mathrm{~h}$ at $37^{\circ} \mathrm{C}$. After the incubation, potassium acetate $(\mathrm{pH} 5,0.2 \mathrm{M})$ was added to stop the aminoacylation reaction. The reaction mix was then phenol-extracted (Phenol pH 4.5, Roth) and aa-tRNA precipitated with ethanol. After pelleting and removal of ethanol in the speedvac, the tRNA was dissolved in water and purified by FPLC on a strong anion-exchange resin (HiTrap Q HP, GE Healthcare). For elution a linear gradient from $0 \mathrm{M}$ to $1.1 \mathrm{M} \mathrm{NaCl}$ in Buffer $\mathrm{E}$ was used. Total tRNA eluted between $650-850 \mathrm{mM} \mathrm{NaCl}$. The tRNA-containing fractions were pooled and ethanol precipitated as described above. To remove traces of salt, the tRNA pellet was washed with $80 \%$ cold ethanol, dried in the speed vac, dissolved in water and stored in aliquots at $-80^{\circ} \mathrm{C}$.

\section{Preparation of mRNA}

The gene prm C coding for the protein methyl transferase PrmC (ECBD_2409, 834 bp, 277 aa, Map position: 2,527,310 <- 2,528,143] (55.29 centisomes)) was amplified from genomic DNA of E. coli BL21 DE3 by colony PCR using the primers "oliPrmCUP" 5'GTCCGAGCAGGACATATGGAATATCAA-3" and "oliPrmCLOW" 5'-GCAGTGTAG AAAAACCTCGAGTTGATAAT-3', digested with NdeI and XhoI (New England Biolabs) and ligated into a pET-24a Vector (Novagen). Transcription templates were generated by PCR amplification of the gene including parts of the pET24 5'UTR using the primers “oliPrmCT7UP” 5'-GATCCCGCGAAATTAATACGACTC-3' and oliPrmC277LOW" 5'TTGATAATAGCGGCCGAGCGTT-3'. To generate transcription templates for the preparation of the fluorescent protein marker, various lower primers annealing at distinct sites in the coding region of prmC were used instead of oliPrmC277LOW. Transcription was carried out in Buffer F containing DTT (10 mM), NTPs (3 mM each), GMP (5 mM), DNA template $(10 \%(\mathrm{v} / \mathrm{v}))$, pyrophosphatase $(5 \mathrm{u} / \mathrm{ml})$, RiboLock RNase inhibitor $(1.5 \%$ (v/v), Fermentas), and T7 RNA-polymerase $(0.8 \%(\mathrm{v} / \mathrm{v}))$, at $37^{\circ} \mathrm{C}$ for $3 \mathrm{~h}$. To purify the mRNA from the transcription mix the RNeasy kit (Qiagen) was used according to the manufacturer's protocol. For each mRNA preparation the homogeneity (>95\%) was checked by polyacrylamide gel electrophoresis. 


\section{Formation of ternary complex and intiation complex}

Ternary complex of EF-Tu, GTP, and aa-tRNA was formed by first incubating EFTu.GDP $(200 \mu \mathrm{M})$ with GTP $(2 \mathrm{mM})$, DTT $(2 \mathrm{mM})$, phosophoenol pyruvate $(3 \mathrm{mM})$, and pyruvate kinase $(0.05 \mathrm{mg} / \mathrm{ml})$ in buffer A supplemented with $\mathrm{MgCl}_{2}(3.5 \mathrm{mM})$ for $15 \mathrm{~min}$ at $37^{\circ} \mathrm{C}$. Then, total aa-tRNA $(100 \mu \mathrm{M})$ was added and the incubation was continued for one additional minute. The ternary complex was then stored on ice and diluted with an equal volume of buffer $\mathrm{C}$ just before the experiment.

Initiation complex was formed by incubating $70 \mathrm{~S}$ ribosomes $(0.75 \mu \mathrm{M})$ in presence of IF 1,2 , and $3\left(0.75 \mu \mathrm{M}\right.$ each), mRNA coding for PrmC $(1.5 \mu \mathrm{M})$, and BOF- $\left.{ }^{3} \mathrm{H}\right] \mathrm{Met}-$ $\operatorname{tRNA}^{\mathrm{fMet}}(0.5 \mu \mathrm{M})$ in buffer A supplemented with DTT $(2 \mathrm{mM})$ and GTP $(1 \mathrm{mM})$ for 30 $\min$ at $37^{\circ} \mathrm{C}$, and subsequently stored on ice. Initiation was monitored by nitrocellulose filtration and quantified as the ratio of $\left[{ }^{3} \mathrm{H}\right]$-radioactivity retained on the filter (bound to ribosomes) and the total $\left[{ }^{3} \mathrm{H}\right]$-radioactivity of the sample determined by liquid scintillation counting. Limiting the amount of initiator tRNA allowed to restrict the number of ribosomes per mRNA to $\leq 1$ and to prevent reinitiation. Initiation complexes were diluted with buffer $\mathrm{C}(1: 1)$ to reduce $\mathrm{Mg}^{2+}$ concentration to $3.5 \mathrm{mM}$ immediately before the translation experiments.

\section{Translation of PrmC and analysis of the formation and decay rates of nascent polypeptides}

Translation of full length PrmC was performed as follows: After temperature equilibration for $10 \mathrm{~s}$ at $37^{\circ} \mathrm{C}$, ternary complex $(40 \mu \mathrm{M})$ and EF-G $(2 \mu \mathrm{M})$ were rapidly mixed with $70 \mathrm{~S}$ initiation complexes $(5-40 \mathrm{nM})$ by vigorous vortexing and then incubated at $37^{\circ} \mathrm{C}$. At desired times reactions were stopped either by flash-freezing in liquid nitrogen or by rapid mixing with $\mathrm{NaOH}(330 \mathrm{mM})$. The frozen samples were then thawed on ice in presence of RNase A $(0.4 \mathrm{mg} / \mathrm{ml}$, Fermentas $)$ and digested for $30 \mathrm{~min}$ at $37^{\circ} \mathrm{C}$. For the samples quenched by $\mathrm{NaOH}$, the material was digested at $37^{\circ} \mathrm{C}$ without addition of RNase. Digested and neutralized samples were then incubated in loading buffer $(50 \mathrm{mM}$ Tris- $\mathrm{HCl}$ $\mathrm{pH} 6.8,12 \%(\mathrm{w} / \mathrm{v})$ glycerol, $2 \% \beta$-mercaptoethanol, $4 \% \mathrm{SDS})$ at $40^{\circ} \mathrm{C}$ for $30 \mathrm{~min}$. The translation of fluorescent marker bands was done in essentially the same way as translation of full length PrmC, but using 70S initiation complexes (20 nM) with mRNA coding for Cterminally truncated variants of PrmC. In this case the samples were incubated for $10 \mathrm{~min}$ before the reaction was stopped. 
Tris-Tricine SDS-PAGE was performed according to the protocol described by Schägger and von Jagow (Schägger \& von Jagow, 1987), using a 4\% stacking, 10\% spacer, and $16.5 \%$ separation gel $(49.5 \% \mathrm{~T}, 3 \% \mathrm{C})$. Alternatively, nascent peptides were separated on Tris-Glycine SDS-PAGE (Laemmli, 1970) using gradient gels (4-20\%, Serva). After the electrophoresis the gel was washed in water for $5 \mathrm{~min}$ and scanned on a FLA-9000 fluorescence imager (Fuji) at $50 \mu \mathrm{m}$ resolution. Fluorescence was excited at $473 \mathrm{~nm}$ and detected after passing the LPB (510LP) filter. Analysis of fluorescence band intensities was performed using ImageJ and PeakFit (Systat Software). The size of nascent protein chains was determined using fluorescent protein marker bands as described in the results section. Decay rates of translation intermediates were determined from plots of the intensity versus time using an exponential function in the form $I=\left(I_{0}-I_{\infty}\right) \times \exp \left(-k_{a p p} t\right)+$ $I_{\infty}$, where $I_{0}$ is the intensity at the start of the reaction, $I_{\infty}$ the intensity at infinity, and $k_{\text {app }}$ the apparent decay rate.

\section{Results}

Depending on the translation initiation region of a particular mRNA and the length and sequence of its coding region, initiation can be rate-limiting for protein synthesis (Jacques \& Dreyfus, 1990; Kudla et al, 2009; Laursen et al, 2005; Milon \& Rodnina, 2012). In order to study translation elongation in isolation from other steps of protein synthesis, initiation was carried out with purified components of the initiation machinery in absence of EF-Tu, EF-G, and elongator tRNAs. The concentrations of ribosomes and initiator tRNA were chosen in such a way that the number of ribosomes per mRNA molecule was limited to one. Translation elongation was started by the addition of aminoacyl-tRNAs and elongation factors to the pre-formed initation complex. Nascent polypeptides were fluorescently labeled at the N-terminus, separated by Tris-Tricine SDSPAGE and detected by fluorescence scanning. Nascent chains detected with this method therefore reflected the protein produced by single ribosomes translating the mRNA molecule. Due to the fact that every nascent chain was labeled with exactly one fluorophore at the N-terminus (Gite et al, 2000) the signal was independent of the length and nature of the nascent chain attached. This is an advantage over most conventional detection methods in which the signal is dependent on the length or amino acid composition of the protein. Furthermore, the translation reaction was carried out in HiFibuffer (buffer B), which allowed translation with the rate and error frequencies comparable to those observed in vivo (Gromadski \& Rodnina, 2004). 
Translation was started by the rapid mixing of ternary complex $(40 \mu \mathrm{M})$ and EF-G $(2 \mu \mathrm{M})$ with ribosomes inititated on the mRNA coding for PrmC and carrying BodipyFLMet-tRNA $^{\mathrm{fMet}}\left(\mathrm{BOF}-\mathrm{Met}-\mathrm{tRNA} \mathrm{fMet}^{\mathrm{fm}}\right)$ in the $\mathrm{P}$ site $(20 \mathrm{nM})$. At defined times the translation was stopped and products were separated by gel electrophoresis (Figure 1A, left panel).

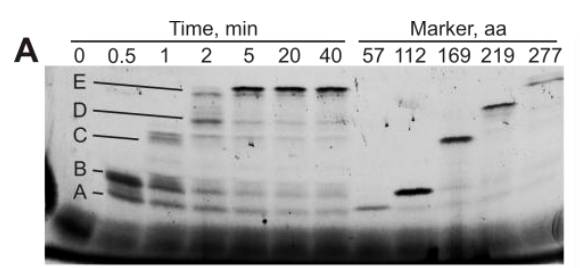

B

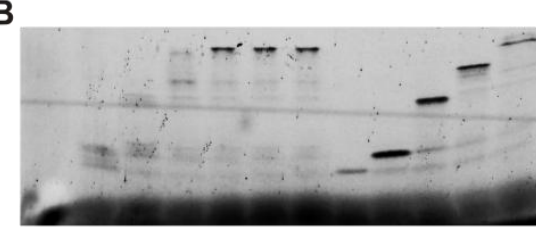

C

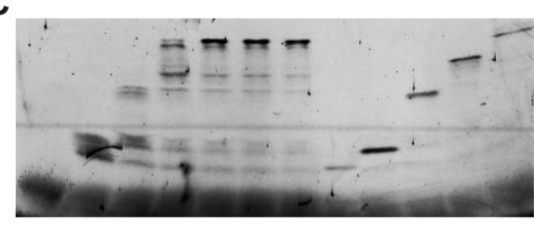

D

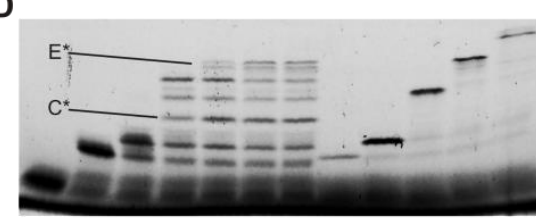

E

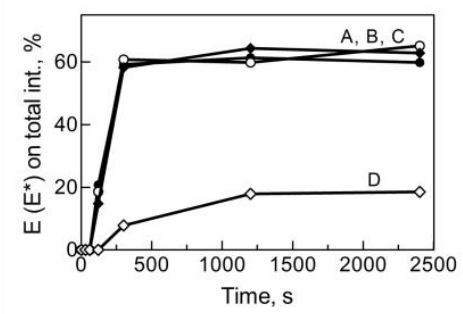

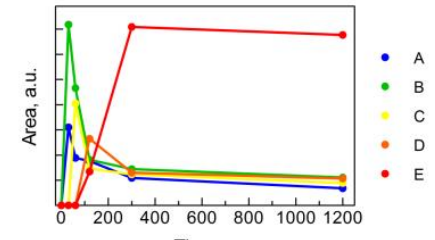

Time, $\mathrm{s}$
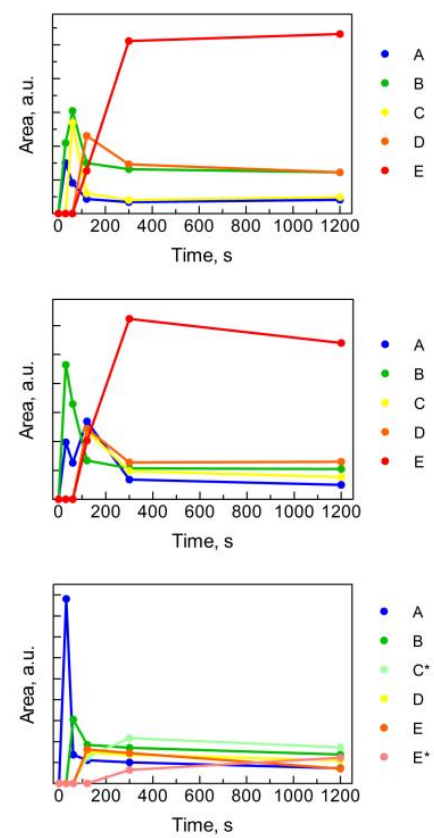

Figure 1 Accumulation of nascent peptides of distinct sizes during translation of full-length PrmC. The reaction was carried out using variable concentrations of initiated ribosomes in the translation reaction (A) 20 $\mathrm{nM}$, (B) $5 \mathrm{nM}$, (C) $10 \mathrm{nM}$, and (D) $40 \mathrm{nM}$. Translation intermediates that were found at all conditions investigated were designated $A-E$. Intermediates $C^{*}$ and $E^{*}$ were only present at $40 \mathrm{nM}$ initiated ribosomes (D). The panels on the left side represent the results of the quantification of the band intensities $A-E^{*}$ (raw data after background subtraction) (E) Formation of the full length product $E$ or the largest product $E^{*}$ over time relative to the other intermediates detected.

The full length product (277 aa) could be detected after $120 \mathrm{~s}$, however also smaller peptides of distinct sizes accumulated during the translation reaction. The peptides were sorted into five groups, which was necessary due to the limited resolution of the gel: $A$ ( 50 aa), $B$ (110-120 aa), $C$ ( 160 aa), D ( 190 aa), and $E$ (270-280 aa, attributed to the 
full length product). The fluorescence intensities of each of those groups and the full length product were quantified and plotted against time (Figure 1A, right panel). Initially (30 s), nascent peptides of groups $A(\sim 50$ aa) and $B(110-120$ aa) were dominant, then at $60 \mathrm{~s}$ peptides of group $C(\sim 160 \mathrm{aa})$ appeared and then disappeared again in favor of peptides of group $D(\sim 190$ aa) and $E(270-280$ aa) at 120 s. At $300 \mathrm{~s}$ the full length product $E$ dominated, which did not change at later time points indicating that the translation reaction was completed. Thus, using nascent peptide analysis, elongating ribosomes could be followed during a single round of mRNA translation. Based on the production of full length product relative to other peptides the translation was found to be $\sim 60 \%$ efficient (i.e. after $300 \mathrm{~s} \sim 60 \%$ of all peptides detected could be attributed to the full length product, Figure 1A, E). Notably, translation elongation was not observed as a uniform process, the simultaneous appearance of peptides of distinct sizes rather indicated that the translation was paused at different sites along the mRNA (Figure 1A, $A-F$ ).

Translational pausing can be caused by limitation of particular tRNA isoacceptors, which is observed e.g. in vivo under conditions of amino acid starvation. In order to identify the causes of translational pausing in the experimental system used here, the translation reaction was carried out using a variable excess of ternary complex over translating ribosomes. This was achieved by variation of the concentration of initiated ribosomes in the translation $\operatorname{mix}(5-40 \mathrm{nM}$, Figure 1B-D), keeping the concentration of ternary complex constant $(40 \mu \mathrm{M})$. The mRNA coding for PrmC comprises 277 codons, meaning that an initiation complex concentration of $5 \mathrm{nM}$ actually represents a concentration of $5 \mathrm{nM} \times 277$ codons $=1.4 \mu \mathrm{M}$ codons, which need to be decoded during the translation experiment. In this case a concentration of $40 \mu \mathrm{M}$ ternary complex corresponds to a 28.5-fold excess of ternary complex at the start of the translation reaction (Figure 1B), which is a simplification as it does not account for the unequal distribution of isoacceptor tRNAs in the total tRNA. In a similar way the concentrations of codons and the excess of ternary complex in the translation experiments were $2.8 \mu \mathrm{M}$ codons and 15 -fold excess, respectively (Figure 1C, $10 \mathrm{nM}$ initiated ribosomes), $5.5 \mu \mathrm{M}$ codons and 7.3-fold excess, respectively (Figure 1A, $20 \mathrm{nM}$ initiated ribosomes), and 11.1 $\mu \mathrm{M}$ codons and 3.6fold excess, respectively (Figure 1D, $40 \mathrm{nM}$ initiated ribosomes). While some groups of peptides $(A-E)$ were present under all experimental conditions tested (Figure 1 A-D), additional nascent peptides ( $C^{*}: \sim 140$ aa, $E^{*}: 210-220$ aa) appeared when the excess of ternary complex was low (3.6-fold, Figure 1D). The amount of full-length product, which was quantified as its intensity over the sum of the intensities of all translation intermediates 
present at a certain time point, was $\sim 60 \%$ over the whole concentration range of $5-20 \mathrm{nM}$ initiated ribosomes i.e. 7.3 - 30-fold excess of ternary complex. For $40 \mathrm{nM}$ initiated ribosomes, or 3.6-fold excess of ternary complex, the full length-product was not synthesized in detectable amounts, and the largest product formed $\left(E^{*}\right)$ was quantified instead (Figure 1E). Taken together, the nascent peptides that appeared only at a low excess of ternary complex (Figure 1D, $C^{*}$ and $E^{*}$ ), were attributed to the depletion of specific isoacceptor tRNAs in the translation mix. The presence of the other five groups of nascent peptides instead (Figure 1A-D, $A-E$ ), was largely unaffected by the excess of ternary complex used. Even though it cannot be ruled out that also these intermediates were a result of an underrepresentation of one or several tRNA isoacceptors, which was not compensated by increasing the excess of ternary complex 8-fold (Figure 1B and D), it might be considered that other factors than tRNA availability played a role in their formation.

In order to study the kinetics of PrmC synthesis and the accumulation of peptides of distinct sizes in more detail, the translation experiment was carried out using the lowest excess of ternary complex (7.3-fold) at which the formation of the final product reached saturation (Figure 1E). This time, a more detailed time course was measured and samples were quenched by rapid mixing with $\mathrm{NaOH}(330 \mathrm{mM})$. The samples were then separated on Tris-Tricine SDS-PAGE along with a marker containing C-terminally truncated variants of PrmC (Figure 2A). After fluorescence scanning 19 nascent peptides were identified including the full length product $(A-S$, Table 1). Peptides shorter than 28 aa were not resolved. From the position of the marker bands the length of the nascent peptides could be determined (Figure 2B, Table 1). The shortest peptide of intermediate size was detected at a length of 51 aa. Longer nascent peptides were distributed over the sequence of the whole protein, located $6-21$ aa apart from each other. Owing to the unequal sizes of the structural domains of PrmC (Yang et al, 2004), most of the translation intermediates therefore could be assigned to the C-terminal domain (87-277 aa), whereas two intermediates were mapped in the linker region (74-86 aa) and two additional ones in the N-terminal domain (1-73 aa) (Figure 2A, Table 1).

The average codon usage frequency of prmC is 20.1 per thousand which corresponds well to the overall frequency of codon usage in E. coli (Nakamura et al, 2000). Despite this global similarity in codon usage, some local regions could be identified in which codon usage deviates from the average (Figure 2C). The length of some of the nascent peptide chains corresponded well to those regions in which codon usage frequency was found to be 
lower than the average, e.g. in the regions from $71-82$ aa, $105-110$ aa, and $130-133$ aa the codon usage frequency was below the average and corresponding intermediates could be detected at 74, 80, 109, and 130 aa (Figure 2C).
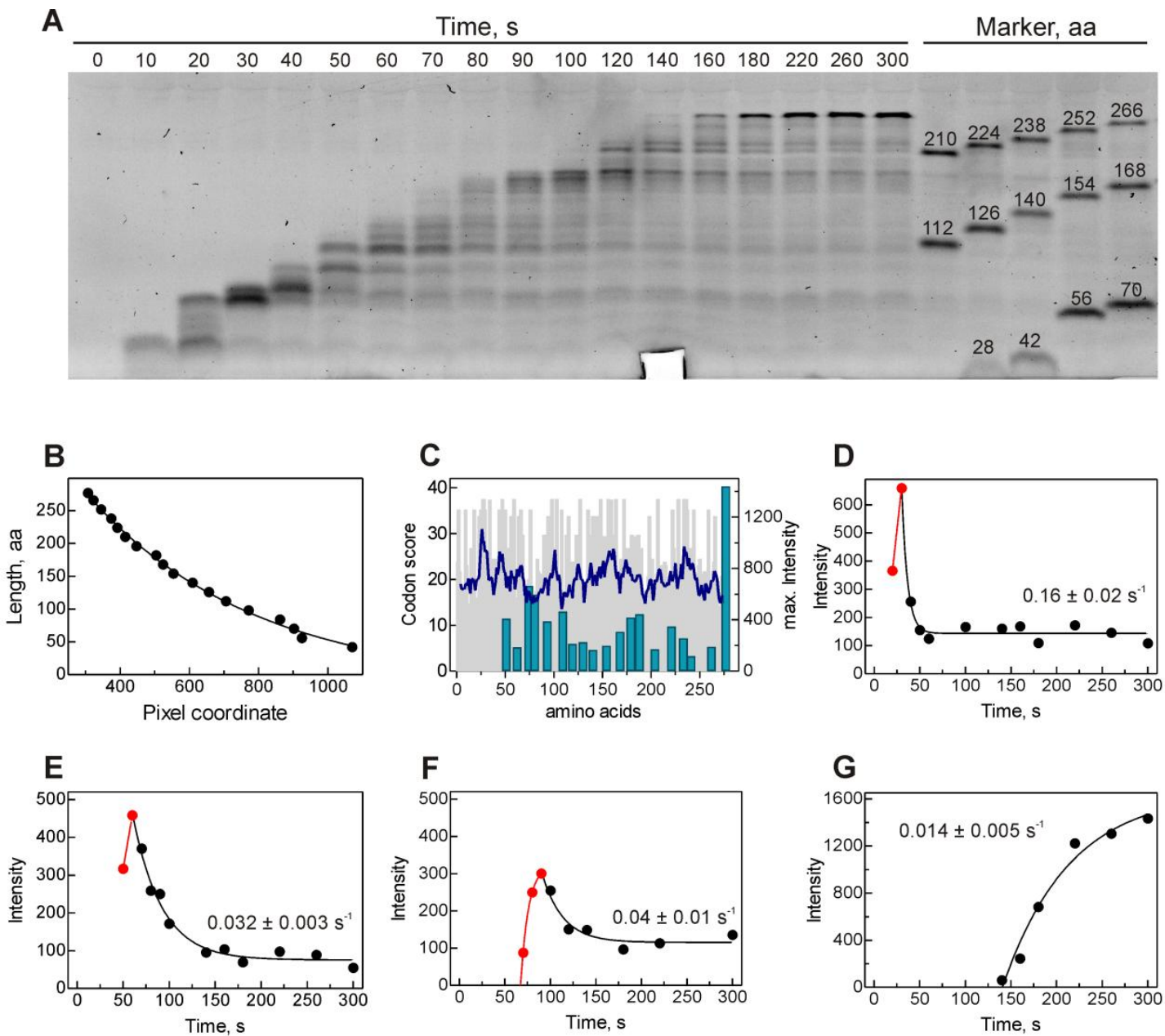

Figure 2 Detailed length analysis of nascent peptides of intermediate sizes and the kinetics of their formation and decay during the translation reaction. (A) Time course of the accumulation of nascent protein chains during translation of the methyltransferase PrmC. Ribosomes initiated with BOF-Met-tRNA ${ }^{\text {fMet }}(20 \mathrm{nM})$ were rapidly mixed with ternary complex EF-Tu.GTP.aa-tRNA $(40 \mu \mathrm{M})$ and EF-G $(2 \mu \mathrm{M})$. Translation was stopped after indicated incubation times by rapid mixing with $\mathrm{NaOH}(330 \mathrm{mM})$ and analyzed by Tris-Tricine SDS-PAGE. (B) Calibration curve for the length determination of the nascent polypeptide chains. The length of the marker bands (in amino acids) was plotted against their position on the gel (in pixels) and analyzed using a simple exponential decay function $\left(\mathrm{Y}=\left(\mathrm{Y}_{0}-\mathrm{Y}_{\infty}\right) \times \exp (-\mathrm{kx})+\mathrm{Y}_{\infty}\right)$, describing the data points in the range between 42 and 277 amino acids $\left(\mathrm{R}^{2}=0.997\right)$. (C) Maximum intensities of nascent peptide chains (blue bars). The codon frequency histogram for prmC (grey bars) and the moving average of codon frequency (blue line) are shown for comparison. (D-G) Time courses of the intensities of different nascent chains and the full length product. The decay rates for nascent polypeptide chains of 74 aa (D), 109 aa (E), 168 aa (F) length, and the formation rate of the full length product 277 aa $(\mathrm{G})$ were analysed by single exponential functions (black lines, see Materials and Methods). The rates for the formation of nascent chains of intermediate sizes (red lines, D-F) could not be determined due to the limited time resolution of the present experiments.

In many cases the low codon usage frequency was caused by the presence of synonymous rare codons. For example in the region 71 - 82 aa, seven amino acids are encoded by lowfrequency codons: Leu71 by UUA (17.4 i.e. frequency per thousand codons) rather than by 
CUG (37.4), Gly73 by GGG (12.3) rather than by GGU (23.7), Arg75 by CGA (4.8) rather than by CGU (15.9), Phe77 by UUC (13.9) rather than by UUU (24.4), Ser79 by UCG (8.2) rather than by AGC (14.3), Leu80 by UUG (12.9) rather than by CUG (37.4), and Leu82 by UUA (17.4) rather than by CUG (37.4). However, a number of intermediates could also be identified in regions where the codon usage frequency was high, e.g. at 140 aa, 154 aa, and at 233 aa.

Table 1 Sizes and formation and decay rates of translation intermediates

\begin{tabular}{lllll}
\hline$\#$ & Length, aa & formation rate, $\mathrm{s}^{-1}$ & decay rate, $\mathrm{s}^{-1}$ & max. intensity \\
\hline A & $51 \pm 2$ & n.d. & $0.22 \pm 0.04$ & 403 \\
$\mathrm{~B}$ & $62 \pm 2$ & n.d. & n.d. & 180 \\
$\mathrm{C}$ & $74 \pm 2$ & n.d. & $0.16 \pm 0.02$ & 659 \\
$\mathrm{D}$ & $80 \pm 2$ & n.d. & n.d. & 590 \\
$\mathrm{E}$ & $93 \pm 1$ & n.d. & $0.04 \pm 0.01$ & 382 \\
$\mathrm{~F}$ & $109 \pm 2$ & n.d. & $0.032 \pm 0.003$ & 459 \\
$\mathrm{G}$ & $119 \pm 2$ & n.d. & $0.03 \pm 0.01$ & 207 \\
$\mathrm{H}$ & $130 \pm 2$ & n.d. & $0.04 \pm 0.01$ & 220 \\
$\mathrm{I}$ & $140 \pm 3$ & n.d. & $0.05 \pm 0.03$ & 160 \\
$\mathrm{~J}$ & $154 \pm 2$ & n.d. & $0.025 \pm 0.007$ & 191 \\
$\mathrm{~K}$ & $168 \pm 3$ & n.d. & $0.044 \pm 0.013$ & 301 \\
$\mathrm{~L}$ & $179 \pm 3$ & n.d. & $0.038 \pm 0.0055$ & 413 \\
$\mathrm{M}$ & $188 \pm 3$ & n.d. & $0.06 \pm 0.05$ & 438 \\
$\mathrm{~N}$ & $204 \pm 2$ & n.d. & n.d. & 164 \\
O & $221 \pm 2$ & n.d. & $0.05 \pm 0.02$ & 343 \\
$\mathrm{P}$ & $233 \pm 2$ & n.d. & $0.06 \pm 0.05$ & 251 \\
$\mathrm{Q}$ & $241 \pm 3$ & n.d. & n.d. & 110 \\
$\mathrm{R}$ & $262 \pm 1$ & n.d. & n.d. & 183 \\
$\mathrm{~S}$ & full length $(277)$ & 0.014 \pm 0.005 & n.d. & 1434 \\
\hline
\end{tabular}

The formation of the full length product occurred at a rate of 1.9 aa s$^{-1}$, which was calculated based on the rate of the appearance of the full length product after a delay of $140 \mathrm{~s}$ during which synthesis already took place (Figure 2G, Table 1). Thus it is comparable to the average translation rates reported in vivo (Sorensen \& Pedersen, 1991); we note that the specific translation rate of PrmC has not been investigated so far. The evaluation of local translation rates was complicated by the fact that the formation rates of nascent peptide chains of intermediate sizes were too fast to be adequately resolved by our method. In general, the intensity of any nascent peptide of intermediate size reached maximal intensity at the first time point or within two or three time points after its initial appearance on the gel (Figure 2D-G). In contrast to the formation rates, the decay rates of the nascent peptides were more defined and showed some variability with rates ranging 
between $0.03 \mathrm{~s}^{-1}$ and $0.22 \mathrm{~s}^{-1}$ (Table 1). Interestingly, the two shortest intermediates for which decay rates could be determined showed the fastest decay rates $\left(0.22\right.$ and $\left.0.16 \mathrm{~s}^{-1}\right)$. The generally observed fast formation of any nascent peptide chain of intermediate size combined with the comparably slow decay rates (Figure 2D-G, Table 1), disfavors a model of a strictly sequential movement of all ribosomes along the mRNA in which the formation of each intermediate is directly resulting from the decay of the preceding intermediate. This was also evident from the fact that at distinct time points more than one nascent chain of intermediate size exists originating from an actively translating ribosome (i.e. excluding intermediates which do not show formation or decay kinetics) (Figure 1A-D, Figure 2A). This suggests that albeit translation was started from synchronized ribosomes, the population of ribosomes became desynchronized during the translation of the mRNA, forming a distribution of productive states of which a significant amount led to the synthesis of the final product. Combining this information leads to a model describing translation elongation in the present system as follows (Figure 3):

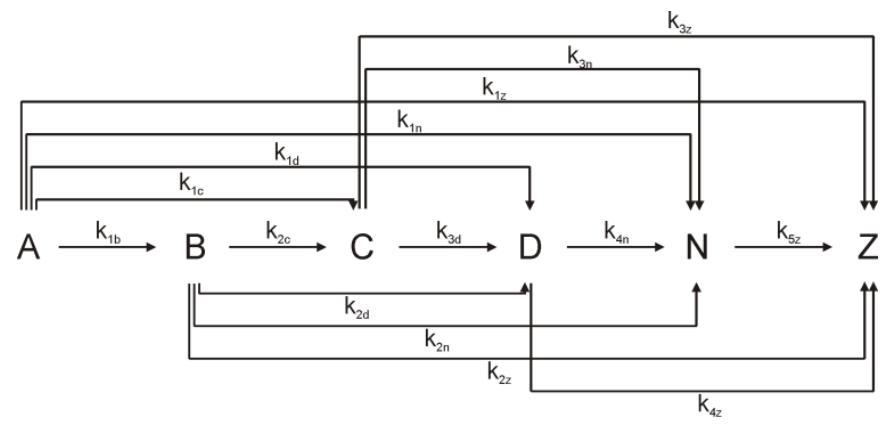

Figure 3 Model for the stochastic, non-uniform movement of ribosomes along an mRNA during synthesis of a single protein. A denotes the starting point (ribosome initiated on an mRNA with an initiator tRNA in the P site), $\mathrm{B}$ to $\mathrm{N}$ are the translation intermediates, $\mathrm{Z}$ is the final product. The rate for the conversion of one intermediate to another is given by $k_{n x}$. Non-productive intermediates, i.e. partitioning from every intermediate to a non-reactive state, has been omitted for clarity.

(i) The translation reaction starts from synchronized ribosomes. (ii) Translation manifests itself in the accumulation of polypeptides of a particular length. (iii) The reaction can only proceed in the forward direction (peptide degradation is excluded). (iv) Pausing along the mRNA is not strictly sequential but rather shows a stochastic distribution. Formation of a translation intermediate is not directly coupled to the decay of the preceding (shorter) intermediate. Accordingly, the decay of a translation intermediate does not directly lead to the formation of the next larger intermediate. The apparent kinetics of the decay of each translation intermediate reflects a sum of all decay rates leading to different larger nascent peptides or the full length product. (v) A certain portion of non-productive translation 
intermediates can accumulate. This might be due to a depletion of certain isoacceptor tRNAs, dissociation of the ribosome from the mRNA or frameshifting. In this model the kinetics leading to the formation of a nascent peptide of a particular size are more complex for larger peptides than for smaller ones, which is due to the number of productive reaction pathways they can originate from. In the same way the decay kinetics are more complex for smaller peptides than for larger ones. This might help to explain the observed tendency for the faster decay rates of shorter peptides (Table 1), simply by the larger number of productive reaction pathways for early intermediates. It should also be considered that the depletion of ternary complex at later time points might cause similar effects, even though the experiments done in different excess of ternary complex (Figure 1) may argue against this notion.

\section{Discussion}

Here we have studied translation elongation kinetics of ribosomes in a minimal system excluding influences from transcription of the mRNA, formation of polysomes and rates of tRNA aminoacylation, during a single round of translation elongation on a natural full length mRNA. The approach presented here allows studies of the elongation kinetics of synchronized ribosomes without the need to stall ribosomes on a unique codon in the open reading frame by depletion of a particular isoacceptor tRNA (Endoh et al, 2012) simply by starting the translation from initiated ribosomes.

Using this system for translation of the model protein PrmC, we find that translation is attenuated at distinct sites resulting in the accumulation of peptides of intermediate sizes (Figure 1 and 2). While some of these intermediates can be attributed to the depletion of tRNAs (Figure 1D), others are largely unaffected by the excess of tRNA used (Figure 1AC, Figure 2A) and might therefore be influenced by different factors. Many of the intermediates are located in areas where the codon usage frequency is low (Figure 2C), which can result in longer arrival times of cognate rare tRNAs, and consequently cause ribosomal pausing. However, this correlation does not seem perfect, because a number of translation intermediates are located in regions where the average codon usage frequency is high (Figure 2C). Additional factors might therefore play a role like translational pausing caused by internal Shine-Dalgarno like sequences (Li et al, 2012). Two Shine-Dalgarno like sequences of the GGXGG-type, can be found in prmC at the positions of codons encoding the amino acids 98-100 and 231-232. Furthermore, the slow decoding of prolines (Pavlov et al, 2009) might also affect local translation rates of PrmC. Especially the proline 
residues at positions 184 and 185, which have a critical function for catalytic activity of PrmC (Schubert et al, 2003), might contribute to translational pausing because an additive effect could be expected. Indeed a corresponding intermediate at $188 \pm 3$ aa is detected (Figure 2A,C).

The observed uncoupling of the formation rate of a nascent peptide chain of a distinct size and the consumption rate of the preceding smaller intermediate (Figure 2C-G, Table 1) indicates that translating ribosomes move along the mRNA molecule in a stochastic manner (Figure 3). The stochastic nature of translation elongation has been investigated in theoretical studies (Garai et al, 2009; Hiernaux, 1974; Mitarai et al, 2008), but so far has not been directly accessible by methods, which observe multiple rounds of translation elongation or provide snapshots of the ribosome distribution along natural mRNAs without a detailed time resolution (Ingolia et al, 2009; Varenne et al, 1984; Wolin \& Walter, 1988). An exception are single molecule approaches, in which the stochastic nature of translation elongation is apparent, but so far was only observed in detail for single rounds of translation elongation (Blanchard et al, 2004; Munro et al, 2010; Whitford et al, 2010), or during translation of short model mRNAs (Qu et al, 2011; Wen et al, 2008). A central concept in models describing the stochastic behavior of translation is that the fundamental steps of elongation are governed by thermal fluctuations, with the translating ribosome acting as a Brownian motor (Fischer et al, 2010; Rodnina et al, 1997; Rodnina \& Wintermeyer, 2011). The main characteristic of a Brownian motor is the conversion of Brownian motions (e.g. thermal fluctuations) in combination with external input signals (e.g. GTP hydrolysis) into a directed motion of particles at a submicrometer scale (Hänggi \& Marchesoni, 2009). Due to this intrinsic stochasticity even the translation of a hypothetical homogeneous mRNA is supposed to be non-uniform and result in a distribution of different states in a population of ribosomes (Garai et al, 2009). Similar to this, diffusion of ternary complex through the medium is subject to random diffusion or Brownian motion. Due to the variation in tRNA abundance and the associated differences in arrival times of the cognate ternary complex at the ribosome (Dong et al, 1996; Fluitt et al, 2007; Ikemura, 1985) decoding of different codons might occur at variable rates, the individual decoding times of each codon following a probability distribution. In that sense, the behavior of the ribosomes studied here might be one of the first examples of directly observed stochastic movement of elongating ribosomes during translation of a full length natural mRNA in a single turnover experiment. Given the complexity of factors that contribute to the stochastic behavior of translating ribosomes, even in the minimal system 
studied here, it seems difficult to make precise assignments of individual contributions for each of the effects described above.

In a physiological context, the stochastic behavior of ribosomes might contribute to a phenomenon known as gene-intrinsic noise (Kaern et al, 2005). Gene-intrinsic noise can be defined as the variation in the expression levels of two identical genes under the control of identical promoters in single cells observed over a large population (Elowitz et al, 2002), and is a general concept used to explain phenotypic variations in populations of genetically identical organisms. Despite the obvious negative effects fluctuations in intracellular protein levels might have, it can represent an important advantage which equips cellular populations with a certain degree of robustness and allows them to adapt to dynamically changing environmental stress conditions like exposure to antibiotics (Lee et al, 2010). It has been shown that the strength of gene-intrinsic noise is rather insensitive to transcription, but directly proportional to translation efficiency and protein abundance (Ozbudak et al, 2002). Given the results obtained in our experimental system it seems likely that translation elongation represents a major source of gene-intrinsic noise in protein expression. The stochastic behavior during translation elongation affects local and global translation rates (Figure 2, Table 1) and might therefore represent a limitation to the overall translation efficiency and protein yield. Finally, it might have important implications for all processes that occur co-translationally and in which the regulation of translation rates is supposed to play an important functional role, such as protein folding (Jha \& Komar, 2011; Komar, 2009), or metabolic gene regulation (Yanofsky, 2004). It suggests that the regulation of translation kinetics needs to be robust enough to cope with a significant amount of noise inherent to the system and might explain why effective codonguided translational pausing might rely on clusters of rare codons, rather than on single rare codons (Clarke \& Clark, 2008). 


\title{
CHAPTER IV: REAL-TIME CO-TRANSLATIONAL FOLDING OF THE N- TERMINAL DOMAIN OF PROTEIN METHYLTRANSFERASE PrmC
}

\begin{abstract}
Protein folding can occur co-translationally while the nascent polypeptide chain is synthesized on the ribosome. The rate of polypeptide elongation may therefore serve as a kinetic guide for co-translational protein folding events. Here we have studied cotranslational folding of the $\mathrm{N}$-terminal domain of PrmC, a protein methyltransferase responsible for the correct function of translational release factors. We have monitored distance changes between two fluorescently labeled amino acids, incorporated at two distinct positions of the polypeptide by fluorescence resonance energy transfer (FRET) in real time. An efficient FRET signal indicating the formation of tertiary structure elements is observed at an early stage, before the synthesis of the first $\sim 110$ amino acids is completed. Our results favor the notion that folding of the $\mathrm{N}$-terminal domain is an early event in the co-translational folding pathway of PrmC. The approach used in this study might be a generally useful strategy to monitor the kinetics of co-translational protein folding events on the ribosome.
\end{abstract}

\section{Introduction}

Many proteins obtain their native tertiary structures while they are synthesized on the ribosome (Cabrita et al, 2010). This process involves a sequential folding of the nascent polypeptide chain which is dominated by the vectorial nature of translation elongation. In many cases co-translational protein folding is more efficient than protein refolding in vitro and can result in increased protein folding rates and higher yields of correctly folded protein (Fedorov \& Baldwin, 1999; Kolb et al, 1994). Furthermore, protein folding on the ribosome is modulated by the structural constraints of the ribosomal tunnel which is $\sim 100$ $\AA$ long, 10 - $20 \AA$ wide, and can accommodate about 30 - 40 amino acids of a nascent peptide chain (Ban et al, 2000). While the tunnel allows the formation of $\alpha$-helices, it can prevent the participation of nascent protein chains in the formation of more complex tertiary structures (Lu \& Deutsch, 2005; Voss et al, 2006; Woolhead et al, 2004). Due to the sequential nature of co-translational protein folding, the rate of polypeptide chain elongation is an important parameter for the process, as it can be rate-limiting for the formation of secondary and tertiary structures elements (Cabrita et al, 2010; Fedorov \& Baldwin, 1997). Furthermore, transient pausing of translation elongation may provide a 
time delay which is necessary for correct folding of distinct parts of the emerging polypeptide chain (Kimchi-Sarfaty et al, 2007; Komar, 2009; Zhang et al, 2009). Moreover, a global reduction of translation rates was shown to facilitate folding of eukaryotic proteins during heterologous expression in prokaryotic cells (Siller et al, 2010). In general, co-translational protein folding might represent a mechanism to prevent misfolding and aggregation of nascent multidomain proteins (Fedorov \& Baldwin, 1997; Hartl et al, 2011), which is fine-tuned by the rate of translation elongation.

So far co-translational protein folding was mainly studied using stalled ribosome nascent chain complexes (Cabrita et al, 2009; Ellis et al, 2008; Khushoo et al, 2011; Komar, 2009; Woolhead et al, 2004), and thus the role of translation rates in the process remained unclear. In order to analyze the potentially fine-balanced effects of translation elongation rates on co-translational protein folding, experimental approaches are required which allow monitoring of nascent chain folding in the millisecond-second range. Cotranslational incorporation of fluorescent amino acids has been used to measure distances in the nascent polypeptide chains of stalled ribosomal complexes by FRET (Khushoo et al, 2011; Woolhead et al, 2004). Here we present an extension of this approach by using presteady state kinetics in order to measure co-translational protein folding on the ribosome in real time.

As a model for our studies we used the N-terminal domain of PrmC (Protein release factor methylation C, formerly HemK) which is an S-adenosyl-L-methionine-dependent protein methyltransferase, catalyzing N5-methylation of a highly conserved glutamine found in the GGQ motif of release factors 1 and 2, and is important for the catalytic activity of peptide chain release (Dincbas-Renqvist et al, 2000; Heurgue-Hamard et al, 2002). PrmC (277 amino acids) is composed of two domains, an N-terminal five-helix bundle (res. 2-73) and a C-terminal domain (87-276) which is a seven-stranded $\beta$-sheet surrounded by three $\alpha$-helices on each side ((Yang et al, 2004), Figure 1). While the carboxy-terminal domain harbors the active site of the protein responsible for release factor methylation, the $\mathrm{NH}_{2}$-terminal domain is important for binding of the release factor (Graille et al, 2005). 


\section{Materials and Methods}

All chemicals were purchased from Merck, Sigma Aldrich, and Life Technologies.

\section{Buffers}

Buffer A Tris- $\mathrm{HCl}(50 \mathrm{mM}) \mathrm{pH} 7.5, \mathrm{NH}_{4} \mathrm{Cl}(70 \mathrm{mM}), \mathrm{KCl}(30 \mathrm{mM}), \mathrm{MgCl}_{2}(7 \mathrm{mM})$

Buffer B Tris- $\mathrm{HCl}(50 \mathrm{mM})$ pH 7.5, $\mathrm{NH}_{4} \mathrm{Cl}(70 \mathrm{mM}), \mathrm{KCl}(30 \mathrm{mM}), \mathrm{MgCl}_{2}$ (3.5 $\mathrm{mM})$, spermidine $(0.5 \mathrm{mM})$, putrescine $(8 \mathrm{mM})$, DTT $(2 \mathrm{mM})$

Buffer C Tris- $\mathrm{HCl}(50 \mathrm{mM}) \mathrm{pH} 7.5, \mathrm{NH}_{4} \mathrm{Cl}(70 \mathrm{mM}), \mathrm{KCl}(30 \mathrm{mM})$, spermidine (1 $\mathrm{mM})$, putrescine $(16 \mathrm{mM})$, DTT $(4 \mathrm{mM})$

All buffers were $\mathrm{pH}$ adjusted at room temperature and filtered through $0.2 \mu \mathrm{m}$ cellulose acetate filters (Sartorius Stedim).

\section{Preparation of components used for in vitro translation}

70S ribosomes from E. coli MRE 600, initiation factors, and EF-G were prepared as described (Milon et al, 2007; Rodnina et al, 1999; Rodnina \& Wintermeyer, 1995). EF-Tu containing a C-terminal oligohistidine tag was expressed and purified according to an established protocol (Wieden et al, 2002). fMet-tRNA ${ }^{\mathrm{fMet}}$, $\alpha$ BodipyFL-Met-tRNA ${ }^{\mathrm{fMet}}$ (BOF-Met-tRNA $^{\mathrm{fMet}}$ ), Lys-tRNA ${ }^{\text {Lys }}$, and $\varepsilon_{N_{2}}$-Bodipy576/589-Lys-tRNA $^{\text {Lys }}$ (BOP-Lys$\mathrm{tRNA}^{\mathrm{Lys}}$ ) were prepared according to the protocol detailed elsewhere (Chapter II). Total aa-tRNA was prepared as described (Chapter III), except that lysine was not added to the aminoacylation mixture. mRNA was prepared from the prmC gene of E. coli BL21 DE3 (ECBD_2409) as described (Chapter III), with the exception that two tryptophan codons were changed to phenylalanine codons (W6F and $\mathrm{W} 78 \mathrm{~F}$ ) by quick change mutagenesis.

\section{Formation of initiation and ternary complexes}

Initiation complexes were formed as described previously (Chapter III), except that mRNAs coding for the 42 and 112 amino acids long $\mathrm{N}$-terminal sequence of PrmC were used. Binary complexes of EF-Tu and GTP were formed by first incubating EF-Tu-GDP $(100 \mu \mathrm{M})$ with GTP $(2 \mathrm{mM})$, DTT $(2 \mathrm{mM})$, phosphoenolpyruvate $(3 \mathrm{mM})$, and pyruvate kinase $(0.05 \mathrm{mg} / \mathrm{ml})$ in buffer A supplemented with $\mathrm{MgCl}_{2}(3.5 \mathrm{mM})$ for $15 \mathrm{~min}$ at $37^{\circ} \mathrm{C}$ and then an equal volume of buffer $\mathrm{C}$ was added. Ternary complex was formed in buffer $\mathrm{B}$ by incubating the binary complex $(45 \mu \mathrm{M})$, BOP-Lys-tRNA ${ }^{\text {Lys }}$ or Lys-tRNA ${ }^{\text {Lys }}(0.2 \mu \mathrm{M})$, and total aa-tRNA without lysine $(20 \mu \mathrm{M})$ for one minute at $37^{\circ} \mathrm{C}$. 


\section{Analysis of nascent polypeptide formation and FRET changes during the translation of the N-terminal domain of PrmC}

After equilibration for $10 \mathrm{~s}$ at $37^{\circ} \mathrm{C}$, ternary complex $(10 \mu \mathrm{M})$ containing BOP-LystRNA $^{\text {Lys }}$ or Lys-tRNA ${ }^{\text {Lys }}$ and EF-G $(2 \mu \mathrm{M})$ were rapidly mixed by vigorous vortexing with $70 S$ initiation complexes $(20 \mathrm{nM})$ containing fMet-tRNA ${ }^{\mathrm{fMet}}$ or BOF-Met-tRNA ${ }^{\mathrm{fMet}}$ and mRNA coding for the 42 or $112 \mathrm{~N}$-terminal amino acids of PrmC, and incubated at $37^{\circ} \mathrm{C}$. At desired times the reaction was stopped by flash freezing in liquid nitrogen. The frozen samples were thawed on ice in the presence of RNase A $(0.4 \mathrm{mg} / \mathrm{ml}$, Fermentas $)$ and digested for $30 \mathrm{~min}$ at $37^{\circ} \mathrm{C}$. After digestion samples were incubated in loading buffer (50 $\mathrm{mM}$ Tris- $\mathrm{HCl} \mathrm{pH} 6.8,12 \%(\mathrm{w} / \mathrm{v})$ glycerol, $2 \% \beta$-mercaptoethanol, $4 \%$ SDS, $0.05 \%$ Coomassie blue G-250) for $30 \mathrm{~min}$ at $40^{\circ} \mathrm{C}$. Tris-tricine SDS-PAGE was performed according to the published protocol (Schägger \& von Jagow, 1987), using a 4\% stacking, $10 \%$ spacer, and $16.5 \%$ separation gel, where the concentration of bisacrylamide relative to the total concentration of acrylamide and bisacrylamide was 3\%. The gel was washed in water for $5 \mathrm{~min}$ and scanned on a FLA-9000 fluorescence imager (Fuji) at $50 \mu \mathrm{m}$ resolution. For the detection of Bodipy FL, the fluorescence was excited at $473 \mathrm{~nm}$ and monitored after passing a LPB (510LP) filter, Bodipy 576/589 fluorescence was excited at $532 \mathrm{~nm}$ and monitored after passing a LPG (575LP) filter.

In parallel to the analysis of the nascent peptides, fluorescence changes of BodipyFL and Bodipy576/589 during translation were monitored in a SX-20MV stopped-flow apparatus (Applied Photophysics, Leatherhead, UK). The excitation wavelength was set to $470 \mathrm{~nm}$ and the emission of BodipyFL and Bodipy576/589 fluorescence was measured simultaneously in two channels after passing through a 500-nm or a 590-nm cutoff filter, respectively. FRET signals ( $\mathrm{F}_{\mathrm{FRET}}$ ) were obtained from the fluorescence changes detected in the acceptor channel (590-nm cutoff filter) by subtracting the fluorescence signal in presence of the fluorescence donor $\left(\mathrm{F}_{\mathrm{D}}\right.$, BodipyFL) and in presence of the fluorescence acceptor $\left(\mathrm{F}_{\mathrm{A}}\right.$, Bodipy576/589) from the signal in presence of both, fluorescence donor and acceptor $\left(\mathrm{F}_{\mathrm{DA}} ; \mathrm{F}_{\mathrm{FRET}}=\mathrm{F}_{\mathrm{DA}}-\mathrm{F}_{\mathrm{D}}-\mathrm{F}_{\mathrm{A}}\right)$, and then aligned by adjusting an offset for ease of comparison. The rates of the FRET changes were analyzed by fitting the experimental data to exponential functions in the form $F_{\text {FRET }}=F_{\infty}+A_{1} \times \exp \left(-k_{a p p 1} \times t\right)+A_{2} \times \exp \left(-k_{a p p} \times t\right)$, where $\mathrm{A}_{1}$ and $\mathrm{A}_{2}$ are FRET amplitudes, $\mathrm{k}_{\mathrm{app} 1}$ and $\mathrm{k}_{\mathrm{app} 2}$ are apparent rates, and $\mathrm{F}_{\infty}$ is the final FRET level. 


\section{Results}

Fluorescence resonance energy transfer is the excited state energy transfer from a donor fluorophore to an acceptor fluorophore without the emission of a photon. FRET efficiency $\left(\mathrm{E}_{\mathrm{FRET}}\right)$ is highly dependent on the distance between the donor and acceptor fluorophores $\left(\mathrm{E}_{\mathrm{FRET}}=\mathrm{R}_{0}{ }^{6} /\left(\mathrm{R}_{0}{ }^{6}+\mathrm{R}^{6}\right)\right.$, where $\mathrm{R}_{0}$ is the distance at which $\mathrm{E}_{\mathrm{FRET}}$ is 0.5 . The Förster radius of the FRET pair BodipyFL/Bodipy576/589 used in this study is $54 \AA$ assuming a random orientation of both fluorophores. In order to measure folding of the Nterminal domain of PrmC, the fluorescently labeled amino acids BodipyFL-methinonie (BOF-Met) and Bodipy576/589-Lysine (BOP-Lys), were incorporated at two positions of the N-terminal domain of PrmC via BOF-Met-tRNA ${ }^{\text {fMet }}$ and BOP-Lys-tRNA ${ }^{\text {Lys }}$, respectively. While BOF-Met replaced the N-terminal methionine, BOP-Lys was incorporated at position 34 replacing a naturally occurring, non-conserved lysine residue located at the N-terminal side of the third $\alpha$-helix (Graille et al, 2005). In the folded protein both positions are located about $15 \AA$ apart (Figure 1), which should result in a FRET efficiency of close to 1 , while in the unfolded protein the distance should be considerably larger and result in an overall lower FRET.

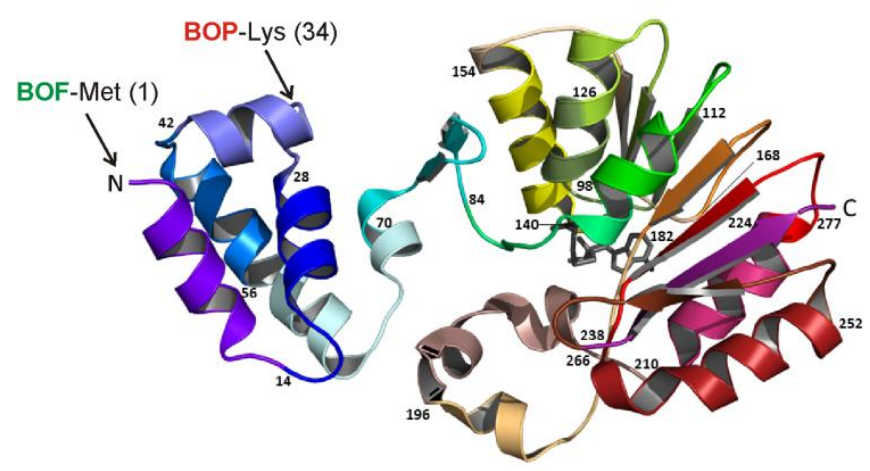

Figure 1 Structure of the methyltransferase PrmC from E. coli ((Yang et al, 2004) PDB code: 1T43). The Nterminal domain comprises a five-helix bundle (residues 2-73) and is connected by a linker region (residues 74-86) to the C-terminal domain (87-267) which is a seven-stranded $\beta$-sheet surrounded by three $\alpha$-helices on each side. Sites for incorporation of the fluorescent non-canonical amino acids, BOF-Met and BOP-Lys in the N-terminal domain of PrmC are indicated by arrows.

To start translation, ternary complex including total aa-tRNA $(10 \mu \mathrm{M})$, Lys-tRNA ${ }^{\text {Lys }}$ or BOP-Lys-tRNA ${ }^{\text {Lys }}(0.1 \mu \mathrm{M})$, and EF-G $(2 \mu \mathrm{M})$ were rapidly mixed with initiation complexes formed with either fMet-tRNA ${ }^{\text {fMet }}$ or BOF-Met-tRNA ${ }^{\text {fMet }}$ and mRNAs coding for N-terminal fragments of PrmC. Fluorescence stopped-flow analysis and nascent peptide chain analysis by SDS-PAGE were carried out in parallel using the same mixtures of ternary complex and initiation complex to minimize pipetting errors. To test whether both dyes were efficiently incorporated into nascent peptide chains of PrmC, a construct of 
42 amino acid length, designated PrmC 42, was translated in the presence of BOF-Met$\mathrm{tRNA}^{\mathrm{fMet}}$ and BOP-Lys-tRNA ${ }^{\mathrm{Lys}}$. The full-length product started to accumulate after $10 \mathrm{~s}$, and the synthesis was essentially completed after $30 \mathrm{~s}$ (Figure 2A, upper panel).
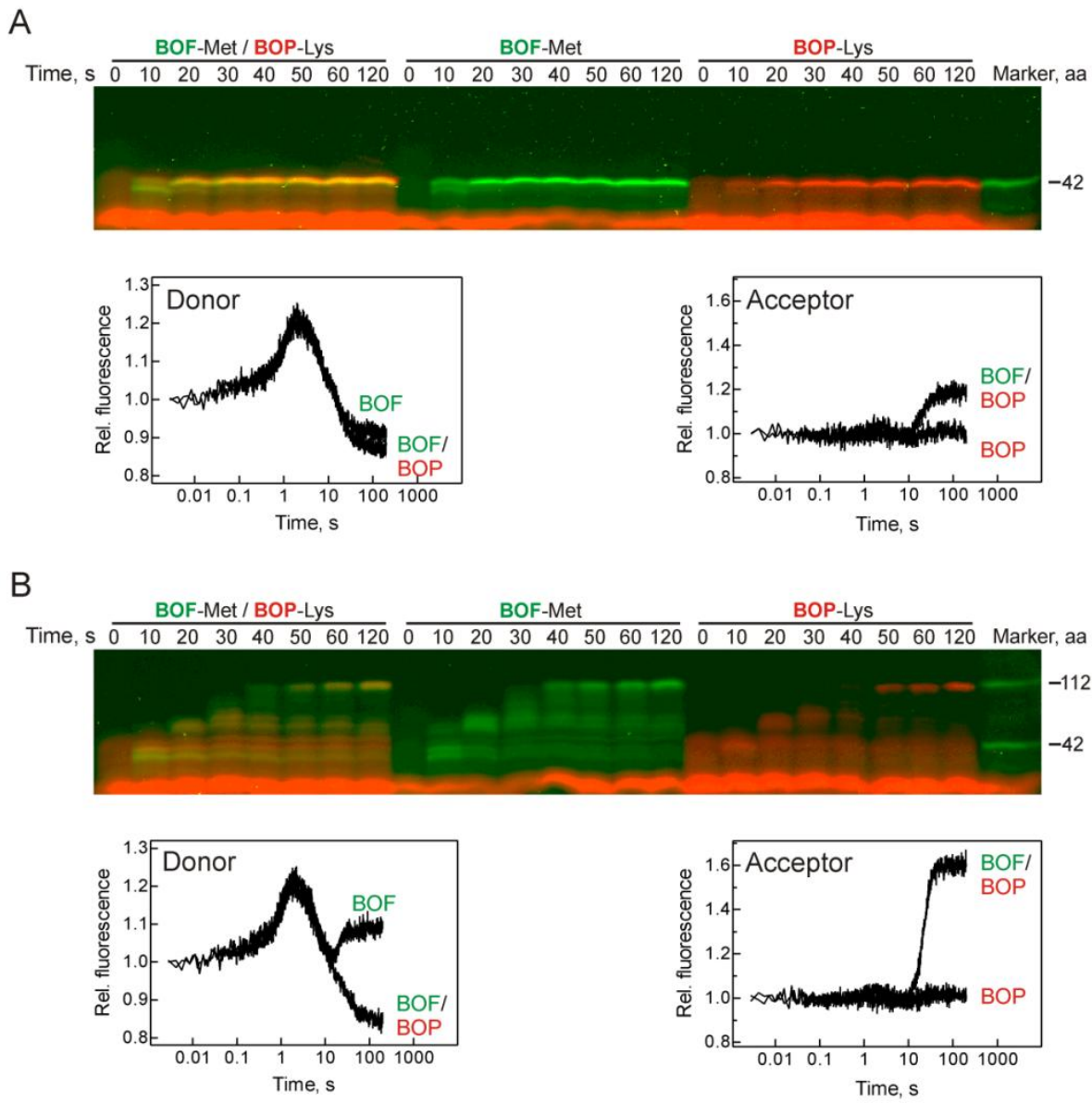

Figure 2 Incorporation and fluorescence changes of donor (BOF) and acceptor (BOP) fluorophores in nascent peptide chains of PrmC. (A) Upper panel: Accumulation of nascent chains during the translation of the N-terminal 42 amino acids of PrmC. Ribosomes were either initiated with BOF-Met-tRNA ${ }^{\text {fMet }}$ (left and middle), or fMet-tRNA ${ }^{\mathrm{fMet}}$ (right), while ternary complexes were either supplemented with BOP-LystRNA $^{\text {Lys }}$ (left and right) or Lys-tRNA ${ }^{\text {Lys }}$ (middle). The fluorescence of Bodipy FL (BOF) was excited at $473 \mathrm{~nm}$ and monitored after passing a 510-nm longpass filter (shown in green), while the fluorescence of Bodipy 576/589 (BOP) was excited at $532 \mathrm{~nm}$ and monitored after passing a 575-nm longpass filter (red). Incorporation of BOF-Met and BOP-Lys resulted in nascent peptides showing fluorescence of both dyes (yellow). A red fluorescent band at the bottom of each lane resulted from the tracking dye used in the gel loading buffer. Lower left panel: Fluorescence change of BodipyFL upon translation of the 42 aa-long Nterminal sequence of PrmC. Excitation was set to $470 \mathrm{~nm}$ and fluorescence was monitored after passing a 500-nm cutoff filter (donor channel). The experiment was done in the absence (BOF) or presence (BOF/BOP) of Bodipy576/589 as FRET acceptor. Lower right panel: Fluorescence change of Bodipy576/589 in the absence (BOP) or presence (BOF/BOP) of BodipyFL as fluorescence donor. (B) Upper panel: Accumulation of nascent chains during the translation of the N-terminal 112 amino acids of PrmC. The experiment was done as in (A) except that a longer mRNA (coding for 112 aa) was used. Lower panels: Fluorescence change of BodipyFL (left panel) and Bodipy576/589 (right panel) during the translation of the 112 aa long N-terminal part of PrmC in presence or absence of the corresponding FRET donor or acceptor dye.

The full-length product showed fluorescence of both dyes, indicating that BOP-Lys was efficiently incorporated into the nascent peptide during translation elongation. In the 
control experiments, in which either BOF-Met-tRNA ${ }^{\mathrm{fMet}}$ or BOP-Lys-tRNA ${ }^{\text {Lys }}$ were substituted by their corresponding non-fluorescent counterparts fMet-tRNA ${ }^{\text {fMet }}$ and Lys$\mathrm{tRNA}^{\mathrm{Lys}}$, full length product was detected after $10 \mathrm{~s}$ and saturation of the band intensities corresponding to the full length product was reached after $30 \mathrm{~s}$. Thus, the fluorescent reporter groups did not seem to have a significant effect on the translation kinetics, although small effects cannot be excluded, due to the limited time resolution of the experiments. In parallel, the fluorescence changes of BodipyFL (BOF) were monitored in stopped-flow experiments (Figure 2A, lower left panel). The fluorescence signal showed a biphasic change in the absence of Bodipy576/589 (BOP), which can be explained by changes in the environment of the fluorophore during continued translation elongation (Wolf Holtkamp, unpublished results). In the presence of the FRET acceptor (BOP) the fluorescence signal reached saturation at a $\sim 5 \%$ lower level than in the absence of the acceptor, whereas the changes in the initial part of the reaction were similar. The fluorescence of Bodipy576/589 (acceptor) did not change to any significant extent in the absence of BodipyFL (donor) (Figure 2A, lower right panel), however in presence of the donor fluorophore it showed a rapid fluorescence increase of $20 \%$ starting at about $10 \mathrm{~s}$, indicating FRET.

A major part of the 42 amino acid-long nascent chain is likely to be contained in the ribosomal tunnel even if a completely extended peptide chain conformation is assumed. In order to allow the N-terminal domain to completely protrude out of the ribosomal tunnel, the same experiment was repeated with a longer mRNA coding for the N-terminal 112 amino acids of PrmC. In this case, different translation intermediates were observed (Figure 2B, upper panel). The first intermediates, which appeared after $10 \mathrm{~s}$ of the reaction, had a size in the range of 42 amino acids or smaller. During further translation, several larger transient intermediates were observed, while the full-length product started to accumulate after $40 \mathrm{~s}$. After $120 \mathrm{~s}$, the major part of the fluorescence intensity was localized in the band corresponding to the full-length product, indicating that the translation reaction was complete. In the control experiments, for which only single fluorophores were used, formation of full length products occurred with comparable kinetics. The initial phases of translation of PrmC 112 showed a similar fluorescence change of BodipyFL in presence or absence of fluorescence acceptor (BOP), however after $10 \mathrm{~s}$ the signals became dramatically different, with fluorescence decreasing in the presence of the acceptor $(\mathrm{BOF} / \mathrm{BOP})$ and increasing with the donor alone $(\mathrm{BOF})$, such that the final fluorescence level was $25 \%$ different in both cases (Figure 2B, lower left panel). 
At the same time, the fluorescence of Bodipy576/589 (BOP) did not change to any significant extent in the absence of donor, however a large increase of about $60 \%$ was observed after $10 \mathrm{~s}$ in presence of the donor fluorophore (BOF/BOP), indicating an efficient FRET. The difference between the decrease of the donor fluorescence (25\%) and the increase of the acceptor fluorescence $(60 \%)$ can be explained by the experimental setup, in which cutoff-filters were used, meaning that an increase in acceptor fluorescence also contributed to the total fluorescence intensity in the donor channel (500-nm cutoff), while in the acceptor channel (590-nm cutoff) contributions of the donor fluorescence were much smaller. By comparing FRET time courses upon translation of PrmC 42 and PrmC 112, we found that in both cases no FRET signal was observed during the first $10 \mathrm{~s}$ of the translation reaction (Figure 3A).

A

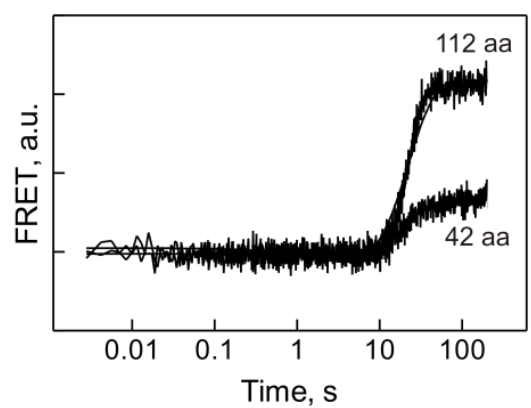

B

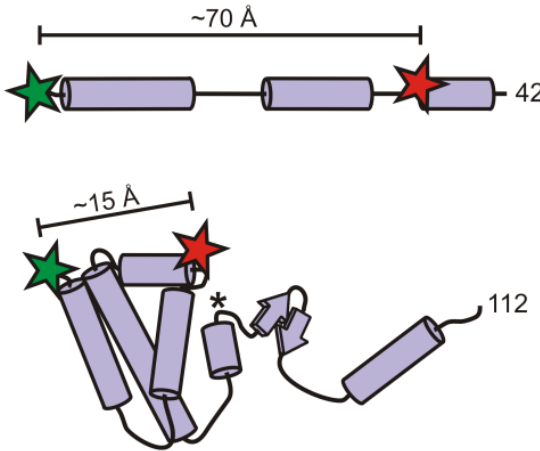

Figure 3 Co-translational FRET changes indicate the compaction of the N-terminal domain of PrmC during its translation on the ribosome. (A) Comparison of co-translational FRET changes of the acceptor fluorophore (BOP) upon translation of the first 42 and 112 amino acids of PrmC. Solid lines show twoexponential fits with $\mathrm{k}_{\mathrm{app} 1}=0.12 \pm 0.09 \mathrm{~s}^{-1}$ and $\mathrm{k}_{\mathrm{app} 2}=0.09 \pm 0.06 \mathrm{~s}^{-1}$ for PrmC 42 and $\mathrm{k}_{\text {app1 }}=0.10 \pm 0.05 \mathrm{~s}^{-1}$ and $\mathrm{k}_{\mathrm{app} 2}=0.09 \pm 0.04 \mathrm{~s}^{-1}$ for PrmC 112, respectively, where $\mathrm{k}_{\text {app1 }}$ corresponds to the duration of the lag phase and $\mathrm{k}_{\text {app2 }}$ represents the apparent rate of the fluorescence increase. (B) Cartoon highlighting the relative positions of donor (green star) and acceptor (red star) fluorophors in the $\mathrm{N}$-terminal domain of PrmC, assuming an extended conformation, comprising only $\alpha$-helices, for the PrmC 42 peptide, and a native fullyfolded tertiary structure for the PrmC 112 peptide. The asterisk indicates a position located $100 \AA$ (the approximate length of the ribosomal tunnel) from the C-terminus of PrmC 112 assuming that the C-terminal end of the nascent chain forms an $\alpha$-helix but remains in an otherwise extended conformation within the peptide tunnel.

The lag phase was followed by a fluorescence increase, which had a similar apparent rate of $0.09 \mathrm{~s}^{-1}$ with PrmC 42 and 112 and in both cases reached saturation after about $45 \mathrm{~s}$. The different intensities of the FRET signals indicate that both labels approached each other more closely in the PrmC 112 peptide, while in the PrmC 42 peptide they remained farther apart from one another (Figure 3B, see discussion). 


\section{Discussion}

Here we present an approach for the real-time monitoring of conformational changes in nascent protein chains during their synthesis on the ribosome. The method is based on the co-translational incorporation of fluorescent amino acids into nascent polypeptide chains (Gite et al, 2000; Johnson, 2005), and allowed us to measure distance changes between residues 1 and 34 of the $\mathrm{N}$-terminal domain of the protein methyltransferase PrmC by FRET. In the translation experiments two different mRNAs coding for the first 42 or 112 amino acids of the N-terminal domain of PrmC were used. Translation of each of those mRNAs resulted in characteristic FRET signal changes which can be divided into three phases: (i) a lag phase, starting form $0.002 \mathrm{~s}$ until $10 \mathrm{~s}$, in which no FRET change is detected, (ii) a phase in which FRET efficiency increases starting from $10 \mathrm{~s}$ until $45 \mathrm{~s}$, and (iii) a phase in which the FRET signals reach a plateau starting from about $45 \mathrm{~s}$ (Figure 3). With some reservations, assuming free mobility of both fluorescent dyes in the nascent polypeptide, and excluding interference of the dyes with the folding process, the observed FRET changes might be interpreted as follows:

The timing of the lag phase, in which no FRET signal is detected, is similar for the translation of PrmC 42 and PrmC 112, suggesting that its end is defined by an event which occurs sometimes before the incorporation of the $43^{\text {rd }}$ amino acid. Thus, the end of the lag phase might either result from the incorporation of the second fluorophore into the nascent chain, or some event that occurs during the synthesis of residues $35-42$. Given the limited time resolution of the fluorescent peptide gels in the current experiments, we cannot answer this question unambiguously at the moment. Assuming $\alpha$-helix formation of the nascent peptide in the ribosomal tunnel (Lu \& Deutsch, 2005; Woolhead et al, 2004), residues 1 and 34 would be located $\sim 70 \AA$ apart from each other (Figure 3B), which could give rise to a moderate FRET efficiency of 0.2. Thus, an increase of FRET efficiency immediately upon incorporation of the second fluorophore might be a plausible explanation for the end of the initial lag phase. During the second phase the FRET efficiency increases at an apparent rate of $0.09 \mathrm{~s}^{-1}$ with both constructs, indicating the occurrence of events which bring the two labels into closer proximity. These events might comprise processes which are either rate-limited by translation elongation or by structural rearrangements of the nascent chain. In both cases this is followed by the third phase in which the FRET efficiencies of PrmC 42 and PrmC 112 saturate at different final levels. As already mentioned, the formation of $\alpha$-helices might bring both fluorophores sufficiently close to account for the observed moderate FRET efficiency in the case of 
PrmC 42 (Figure 3). It is not known if the 42 amino acid long polypeptide would theoretically be able to form a stable tertiary structure by itself, however given that the majority of the nascent chain resides inside of the ribosome, tertiary structure formation will likely be prevented by the constraints of the ribosomal tunnel ( $\mathrm{Lu} \&$ Deutsch, 2005; Voss et al, 2006). Continued translation elongation in case of PrmC 112 is expected to allow a larger part of the nascent chain to exit the ribosomal tunnel. Assuming $\alpha$-helix formation in the C-terminal part of PrmC 112, and given the length of the ribosomal tunnel of $\sim 100 \AA$ (Ban et al, 2000), this would allow residues 1-73 to exit the ribosomal tunnel and participate in tertiary structure formation, which might account for the observed high FRET efficiency with PrmC 112. Interestingly, maximum FRET efficiency is reached before the synthesis of 112 amino acids is fully completed (Figure 2B, Figure 3A), which indicates that both labels approach each other closer than $25 \AA\left(\mathrm{E}_{\mathrm{FRET}}=0.99\right)$, before residues 1-73 completely protrude out of the ribosomal tunnel. This early event which probably involves the formation of tertiary structure elements, might be consistent with the more recent view that the last $20 \AA$ of the ribosomal exit tunnel can already allow the formation of some smaller tertiary structures (O'Brien et al, 2010).

Overall, the observed transient FRET changes are consistent with an early folding of the N-terminal domain of PrmC during continued elongation, in which tertiary interactions are established early as the nascent chain exits the first $\sim 80 \AA$ of the ribosomal tunnel (Figure 3B). Furthermore, folding of the N-terminal domain (residues 1-73) takes place in the absence of the C-terminal domain, consistent with the notion that structural homologs of the N-terminal domain of PrmC, such as domain I of elongation factor TFIIS of RNApolymerase II (Booth et al, 2000) and the EPS15 homology (EH) domain (de Beer et al, 1998), exist as independently folded structural units in solution (Yang et al, 2004). Furthermore, folding of residues 1-73 of PrmC from an unstructured peptide in solution is predicted to occur rapidly, at a rate of $\ln (k f)=11.6 \mathrm{~s}^{-1}$ (Gromiha et al, 2006). The functional importance of the early folding of the PrmC N-terminal domain is not clear, because to our knowledge no unfolding/refolding studies of separate domains or the complete protein have been performed so far. However, in general it could be expected that sequential folding serves as a mechanism to decrease the conformational energy of nascent polypeptide chains during their synthesis on the ribosome and thereby avoid kinetic traps on the folding pathway. Therefore, formation of a structured $\mathrm{N}$-terminal domain at an early stage in the folding pathway of PrmC might serve as a platform to facilitate the folding of the more complex C-terminal domain during continued protein chain elongation. 


\section{REFERENCES}

Adamczyk AJ, Warshel A (2011) Converting structural information into an allosteric-energy-based picture for elongation factor Tu activation by the ribosome. Proc Natl Acad Sci USA 108: 98279832

Agirrezabala X, Schreiner E, Trabuco LG, Lei J, Ortiz-Meoz RF, Schulten K, Green R, Frank J (2011) Structural insights into cognate versus near-cognate discrimination during decoding. EMBO J 30: $1497-1507$

Agris PF (2004) Decoding the genome: a modified view. Nucleic Acids Res 32: 223-238

An S, Musier-Forsyth K (2005) Cys-tRNA(Pro) editing by Haemophilus influenzae YbaK via a novel synthetase. YbaK.tRNA ternary complex. J Biol Chem 280: 34465-34472

Anfinsen CB (1973) Principles that govern the folding of protein chains. Science 181: 223-230

Ban N, Nissen P, Hansen J, Moore PB, Steitz TA (2000) The complete atomic structure of the large ribosomal subunit at 2.4 A resolution. Science 289: 905-920

Bashan A, Yonath A (2008) Correlating ribosome function with high-resolution structures. Trends Microbiol 16: 326-335

Berchtold H, Reshetnikova L, Reiser CO, Schirmer NK, Sprinzl M, Hilgenfeld R (1993) Crystal structure of active elongation factor Tu reveals major domain rearrangements. Nature 365: 126-132

Bergman LW, Kuehl WM (1979) Formation of an intrachain disulfide bond on nascent immunoglobulin light chains. J Biol Chem 254: 8869-8876

Bhuta A, Quiggle K, Ott T, Ringer D, Chladek S (1981) Stereochemical control of ribosomal peptidyltransferase reaction. Role of amino acid side-chain orientation of acceptor substrate. Biochemistry 20: 8-15

Blanchard SC, Gonzalez RL, Kim HD, Chu S, Puglisi JD (2004) tRNA selection and kinetic proofreading in translation. Nat Struct Mol Biol 11: 1008-1014

Booth V, Koth CM, Edwards AM, Arrowsmith CH (2000) Structure of a conserved domain common to the transcription factors TFIIS, elongin A, and CRSP70. J Biol Chem 275: 3126631268

Borgia MB, Borgia A, Best RB, Steward A, Nettels D, Wunderlich B, Schuler B, Clarke J (2011) Single-molecule fluorescence reveals sequence-specific misfolding in multidomain proteins. Nature 474: 662-665

Brandt F, Etchells SA, Ortiz JO, Elcock AH, Hartl FU, Baumeister W (2009) The native 3D organization of bacterial polysomes. Cell 136: 261-271

Brockwell DJ, Radford SE (2007) Intermediates: ubiquitous species on folding energy landscapes? Curr Opin Struct Biol 17: 30-37

Buchan JR, Stansfield I (2007) Halting a cellular production line: responses to ribosomal pausing during translation. Biol Cell 99: 475-487 
Burakovsky DE, Sergiev PV, Steblyanko MA, Kubarenko AV, Konevega AL, Bogdanov AA, Rodnina MV, Dontsova OA (2010) Mutations at the accommodation gate of the ribosome impair RF2-dependent translation termination. RNA 16: 1848-1853

Burmann BM, Schweimer K, Luo X, Wahl MC, Stitt BL, Gottesman ME, Rosch P (2010) A NusE:NusG complex links transcription and translation. Science 328: 501-504

Byrne RT, Konevega AL, Rodnina MV, Antson AA (2010) The crystal structure of unmodified tRNAPhe from Escherichia coli. Nucleic Acids Res 38: 4154-4162

Cabrita LD, Dobson CM, Christodoulou J (2010) Protein folding on the ribosome. Curr Opin Struct Biol 20: 33-45

Cabrita LD, Hsu ST, Launay H, Dobson CM, Christodoulou J (2009) Probing ribosome-nascent chain complexes produced in vivo by NMR spectroscopy. Proc Natl Acad Sci USA 106: 2223922244

Carter AP, Clemons WM, Brodersen DE, Morgan-Warren RJ, Wimberly BT, Ramakrishnan V (2000) Functional insights from the structure of the 30S ribosomal subunit and its interactions with antibiotics. Nature 407: 340-348

Cayama E, Yepez A, Rotondo F, Bandeira E, Ferreras AC, Triana-Alonso FJ (2000) New chromatographic and biochemical strategies for quick preparative isolation of tRNA. Nucleic Acids Res 28: E64

Cech TR (2000) Structural biology. The ribosome is a ribozyme. Science 289: 878-879

Chapeville F, Lipmann F, Von Ehrenstein G, Weisblum B, Ray WJ, Jr., Benzer S (1962) On the role of soluble ribonucleic acid in coding for amino acids. Proc Natl Acad Sci USA 48: 1086-1092

Chapman SJ, Schrader JM, Uhlenbeck OC (2012) Histidine 66 in Escherichia coli elongation factor tu selectively stabilizes aminoacyl-tRNAs. J Biol Chem 287: 1229-1234

Chin JW, Cropp TA, Anderson JC, Mukherji M, Zhang Z, Schultz PG (2003) An expanded eukaryotic genetic code. Science 301: 964-967

Chin JW, Martin AB, King DS, Wang L, Schultz PG (2002) Addition of a photocrosslinking amino acid to the genetic code of Escherichiacoli. Proc Natl Acad Sci USA 99: 11020-11024

Clarke TFt, Clark PL (2008) Rare codons cluster. PLoS One 3: e3412

Cochella L, Green R (2005) An active role for tRNA in decoding beyond codon:anticodon pairing. Science 308: 1178-1180

Cortazzo P, Cervenansky C, Marin M, Reiss C, Ehrlich R, Deana A (2002) Silent mutations affect in vivo protein folding in Escherichia coli. Biochem Biophys Res Commun 293: 537-541

Cowie DB, Cohen GN (1957) Biosynthesis by Escherichia coli of active altered proteins containing selenium instead of sulfur. Biochim Biophys Acta 26: 252-261

Crombie T, Boyle JP, Coggins JR, Brown AJ (1994) The folding of the bifunctional TRP3 protein in yeast is influenced by a translational pause which lies in a region of structural divergence with Escherichia coli indoleglycerol-phosphate synthase. Eur J Biochem 226: 657-664

Crombie T, Swaffield JC, Brown AJ (1992) Protein folding within the cell is influenced by controlled rates of polypeptide elongation. J Mol Biol 228: 7-12 
Dale T, Fahlman RP, Olejniczak M, Uhlenbeck OC (2009) Specificity of the ribosomal A site for aminoacyl-tRNAs. Nucleic Acids Res 37: 1202-1210

Dale T, Sanderson LE, Uhlenbeck OC (2004) The affinity of elongation factor $\mathrm{Tu}$ for an aminoacyl-tRNA is modulated by the esterified amino acid. Biochemistry 43: 6159-6166

Dale T, Uhlenbeck OC (2005a) Amino acid specificity in translation. Trends Biochem Sci 30: 659665

Dale T, Uhlenbeck OC (2005b) Binding of misacylated tRNAs to the ribosomal A site. RNA 11: $1610-1615$

Davies J, Gilbert W, Gorini L (1964) Streptomycin, Suppression, and the Code. Proc Natl Acad Sci USA 51: 883-890

Davis L, Chin JW (2012) Designer proteins: applications of genetic code expansion in cell biology. Nat Rev Mol Cell Biol 13: 168-182

Daviter T, Wieden HJ, Rodnina MV (2003) Essential role of histidine 84 in elongation factor Tu for the chemical step of GTP hydrolysis on the ribosome. J Mol Biol 332: 689-699

De Beer T, Carter RE, Lobel-Rice KE, Sorkin A, Overduin M (1998) Structure and Asn-Pro-Phe binding pocket of the Eps15 homology domain. Science 281: 1357-1360

Demeshkina N, Jenner L, Westhof E, Yusupov M, Yusupova G (2012) A new understanding of the decoding principle on the ribosome. Nature 484: 256-259

Diaconu M, Kothe U, Schlunzen F, Fischer N, Harms JM, Tonevitsky AG, Stark H, Rodnina MV, Wahl MC (2005) Structural basis for the function of the ribosomal L7/12 stalk in factor binding and GTPase activation. Cell 121: 991-1004

Dincbas-Renqvist V, Engstrom A, Mora L, Heurgue-Hamard V, Buckingham R, Ehrenberg M (2000) A post-translational modification in the GGQ motif of RF2 from Escherichia coli stimulates termination of translation. EMBO J 19: 6900-6907

Dittmar KA, Sorensen MA, Elf J, Ehrenberg M, Pan T (2005) Selective charging of tRNA isoacceptors induced by amino-acid starvation. EMBO Rep 6: 151-157

Doi Y, Ohtsuki T, Shimizu Y, Ueda T, Sisido M (2007) Elongation factor Tu mutants expand amino acid tolerance of protein biosynthesis system. J Am Chem Soc 129: 14458-14462

Dong H, Nilsson L, Kurland CG (1996) Co-variation of tRNA abundance and codon usage in Escherichia coli at different growth rates. J Mol Biol 260: 649-663

Doose S, Neuweiler H, Sauer M (2009) Fluorescence quenching by photoinduced electron transfer: A reporter for conformational dynamics of macromolecules. ChemPhysChem 10: 1389-1398

Driscoll DM, Copeland PR (2003) Mechanism and regulation of selenoprotein synthesis. Annu Rev Nutr 23: $17-40$

Drummond DA, Wilke CO (2009) The evolutionary consequences of erroneous protein synthesis. Nat Rev Genet 10: 715-724 
Effraim PR, Wang J, Englander MT, Avins J, Leyh TS, Gonzalez RL, Jr., Cornish VW (2009) Natural amino acids do not require their native tRNAs for efficient selection by the ribosome. Nat Chem Biol 5: 947-953

Elf J, Nilsson D, Tenson T, Ehrenberg M (2003) Selective charging of tRNA isoacceptors explains patterns of codon usage. Science 300: 1718-1722

Ellis JP, Bakke CK, Kirchdoerfer RN, Jungbauer LM, Cavagnero S (2008) Chain dynamics of nascent polypeptides emerging from the ribosome. ACS Chem Biol 3: 555-566

Elowitz MB, Levine AJ, Siggia ED, Swain PS (2002) Stochastic gene expression in a single cell. Science 297: 1183-1186

Endoh T, Kawasaki Y, Sugimoto N (2012) Synchronized translation for detection of temporal stalling of ribosome during single-turnover translation. Anal Chem 84: 857-861

Fedorov AN, Baldwin TO (1997) Cotranslational protein folding. J Biol Chem 272: 32715-32718

Fedorov AN, Baldwin TO (1999) Process of biosynthetic protein folding determines the rapid formation of native structure. J Mol Biol 294: 579-586

Ferbitz L, Maier T, Patzelt H, Bukau B, Deuerling E, Ban N (2004) Trigger factor in complex with the ribosome forms a molecular cradle for nascent proteins. Nature 431: 590-596

Fischer N, Konevega AL, Wintermeyer W, Rodnina MV, Stark H (2010) Ribosome dynamics and tRNA movement by time-resolved electron cryomicroscopy. Nature 466: 329-333

Fluitt A, Pienaar E, Viljoen H (2007) Ribosome kinetics and aa-tRNA competition determine rate and fidelity of peptide synthesis. Comput Biol Chem 31: 335-346

Frydman J, Erdjument-Bromage H, Tempst P, Hartl FU (1999) Co-translational domain folding as the structural basis for the rapid de novo folding of firefly luciferase. Nat Struct Biol 6: 697-705

Garai A, Chowdhury D, Ramakrishnan TV (2009) Fluctuations in protein synthesis from a single RNA template: stochastic kinetics of ribosomes. Phys Rev E Stat Nonlin Soft Matter Phys 79: 011916

Geggier P, Dave R, Feldman MB, Terry DS, Altman RB, Munro JB, Blanchard SC (2010) Conformational sampling of aminoacyl-tRNA during selection on the bacterial ribosome. $J \mathrm{Mol}$ Biol 399: 576-595

Gite S, Mamaev S, Olejnik J, Rothschild K (2000) Ultrasensitive fluorescence-based detection of nascent proteins in gels. Anal Biochem 279: 218-225

Graille M, Heurgue-Hamard V, Champ S, Mora L, Scrima N, Ulryck N, van Tilbeurgh H, Buckingham RH (2005) Molecular basis for bacterial class I release factor methylation by PrmC. Mol Cell 20: 917-927

Gromadski KB, Daviter T, Rodnina MV (2006) A uniform response to mismatches in codonanticodon complexes ensures ribosomal fidelity. Mol Cell 21: 369-377

Gromadski KB, Rodnina MV (2004a) Kinetic determinants of high-fidelity tRNA discrimination on the ribosome. Mol Cell 13: 191-200 
Gromadski KB, Rodnina MV (2004b) Streptomycin interferes with conformational coupling between codon recognition and GTPase activation on the ribosome. Nat Struct Mol Biol 11: 316322

Gromadski KB, Wieden HJ, Rodnina MV (2002) Kinetic mechanism of elongation factor Tscatalyzed nucleotide exchange in elongation factor Tu. Biochemistry 41: 162-169

Gromiha MM, Thangakani AM, Selvaraj S (2006) FOLD-RATE: prediction of protein folding rates from amino acid sequence. Nucleic Acids Res 34: W70-74

Guo J, Melancon CE, 3rd, Lee HS, Groff D, Schultz PG (2009) Evolution of amber suppressor tRNAs for efficient bacterial production of proteins containing nonnatural amino acids. Angew Chem Int Ed Engl 48: 9148-9151

Hancock SM, Uprety R, Deiters A, Chin JW (2010) Expanding the genetic code of yeast for incorporation of diverse unnatural amino acids via a pyrrolysyl-tRNA synthetase/tRNA pair. $J \mathrm{Am}$ Chem Soc 132: 14819-14824

Hänggi P, Marchesoni F (2009) Artificial Brownian motors: Controlling transport on the nanoscale. Rev Mod Phys 81: 387-442

Hartl FU, Bracher A, Hayer-Hartl M (2011) Molecular chaperones in protein folding and proteostasis. Nature 475: $324-332$

Hendrickson WA, Horton JR, LeMaster DM (1990) Selenomethionyl proteins produced for analysis by multiwavelength anomalous diffraction (MAD): a vehicle for direct determination of three-dimensional structure. EMBO J 9: 1665-1672

Heurgue-Hamard V, Champ S, Engstrom A, Ehrenberg M, Buckingham RH (2002) The hemK gene in Escherichia coli encodes the N(5)-glutamine methyltransferase that modifies peptide release factors. $E M B O J$ 21: 769-778

Hiernaux J (1974) On some stochastic models for protein biosynthesis. Biophys Chem 2: 70-75

Hirsh D (1971) Tryptophan transfer RNA as the UGA suppressor. J Mol Biol 58: 439-458

Hirsh D, Gold L (1971) Translation of the UGA triplet in vitro by tryptophan transfer RNA's. J Mol Biol 58: 459-468

Hohsaka T, Kajihara D, Ashizuka Y, Murakami H, Sisido M. (1999) Efficient Incorporation of Nonnatural Amino Acids with Large Aromatic Groups into Streptavidin in In Vitro Protein Synthesizing Systems. J Am Chem Soc Vol. 121, pp. 34-40.

Holmes WM, Hurd RE, Reid BR, Rimerman RA, Hatfield GW (1975) Separation of transfer ribonucleic acid by sepharose chromatography using reverse salt gradients. Proc Natl Acad Sci USA 72: 1068-1071

Hsu ST, Fucini P, Cabrita LD, Launay H, Dobson CM, Christodoulou J (2007) Structure and dynamics of a ribosome-bound nascent chain by NMR spectroscopy. Proc Nat Acad Sci USA 104: $16516-16521$

Ibba M, Söll D (2000) Aminoacyl-tRNA synthesis. Annu Rev Biochem 69: 617-650

Iijima I, Hohsaka T (2009) Position-specific incorporation of fluorescent non-natural amino acids into maltose-binding protein for detection of ligand binding by FRET and fluorescence quenching. Chembiochem 10: 999-1006 
Ikemura T (1985) Codon usage and tRNA content in unicellular and multicellular organisms. $\mathrm{Mol}$ Biol Evol 2: 13-34

Ingolia NT, Ghaemmaghami S, Newman JR, Weissman JS (2009) Genome-wide analysis in vivo of translation with nucleotide resolution using ribosome profiling. Science 324: 218-223

Ito K, Chiba S, Pogliano K (2010) Divergent stalling sequences sense and control cellular physiology. Biochem Biophys Res Commun 393: 1-5

Ito K, Uno M, Nakamura Y (2000) A tripeptide 'anticodon' deciphers stop codons in messenger RNA. Nature 403: 680-684

Jacques N, Dreyfus M (1990) Translation initiation in Escherichia coli: old and new questions. $M o l$ Microbiol 4: 1063-1067

Jenner L, Demeshkina N, Yusupova G, Yusupov M (2010a) Structural rearrangements of the ribosome at the tRNA proofreading step. Nat Struct Mol Biol 17: 1072-1078

Jenner L, Demeshkina N, Yusupova G, Yusupov M (2010b) Structural aspects of messenger RNA reading frame maintenance by the ribosome. Nat Struct Mol Biol 17: 555-560

Jha S, Komar AA (2011) Birth, life and death of nascent polypeptide chains. Biotechnol J 6: 623640

Johnson AE (2005) The co-translational folding and interactions of nascent protein chains: a new approach using fluorescence resonance energy transfer. FEBS Lett 579: 916-920

Johnson JA, Lu YY, Van Deventer JA, Tirrell DA (2010) Residue-specific incorporation of noncanonical amino acids into proteins: recent developments and applications. Curr Opin Chem Biol 14: $774-780$

Julian P, Milon P, Agirrezabala X, Lasso G, Gil D, Rodnina MV, Valle M (2011) The Cryo-EM structure of a complete $30 \mathrm{~S}$ translation initiation complex from Escherichia coli. PLoS biology 9: e1001095

Kaern M, Elston TC, Blake WJ, Collins JJ (2005) Stochasticity in gene expression: from theories to phenotypes. Nat Rev Genet 6: 451-464

Kajihara D, Abe R, Iijima I, Komiyama C, Sisido M, Hohsaka T (2006) FRET analysis of protein conformational change through position-specific incorporation of fluorescent amino acids. Nat Methods 3: 923-929

Kast P, Hennecke H (1991) Amino acid substrate specificity of Escherichia coli phenylalanyltRNA synthetase altered by distinct mutations. J Mol Biol 222: 99-124

Katranidis A, Atta D, Schlesinger R, Nierhaus KH, Choli-Papadopoulou T, Gregor I, Gerrits M, Buldt G, Fitter J (2009) Fast biosynthesis of GFP molecules: a single-molecule fluorescence study. Angew Chem Int Ed Engl 48: 1758-1761

Kelkar DA, Khushoo A, Yang Z, Skach WR (2012) Kinetic analysis of ribosome-bound fluorescent proteins reveals an early, stable, cotranslational folding intermediate. J Biol Chem 287: $2568-2578$

Khushoo A, Yang Z, Johnson AE, Skach WR (2011) Ligand-driven vectorial folding of ribosomebound human CFTR NBD1. Mol Cell 41: 682-692 
Kiick KL, van Hest JC, Tirrell DA (2000) Expanding the Scope of Protein Biosynthesis by Altering the Methionyl-tRNA Synthetase Activity of a Bacterial Expression Host. Angew Chem Int Ed Engl 39: 2148-2152

Kim DF, Green R (1999) Base-pairing between 23S rRNA and tRNA in the ribosomal A site. Mol Cell 4: 859-864

Kim JK, Hollingsworth MJ (1992) Localization of in vivo ribosome pause sites. Anal Biochem 206: $183-188$

Kimchi-Sarfaty C, Oh JM, Kim IW, Sauna ZE, Calcagno AM, Ambudkar SV, Gottesman MM (2007) A "silent" polymorphism in the MDR1 gene changes substrate specificity. Science 315: $525-528$

Kjeldgaard M, Nyborg J (1992) Refined structure of elongation factor EF-Tu from Escherichia coli. J Mol Biol 223: 721-742

Kobayashi T, Takimura T, Sekine R, Kelly VP, Kamata K, Sakamoto K, Nishimura S, Yokoyama S (2005) Structural snapshots of the KMSKS loop rearrangement for amino acid activation by bacterial tyrosyl-tRNA synthetase. J Mol Biol 346: 105-117

Kolb VA, Makeyev EV, Spirin AS (1994) Folding of firefly luciferase during translation in a cellfree system. EMBO J 13: 3631-3637

Komar AA (2007) Genetics. SNPs, silent but not invisible. Science 315: 466-467

Komar AA (2009) A pause for thought along the co-translational folding pathway. Trends Biochem Sci 34: 16-24

Komar AA, Kommer A, Krasheninnikov IA, Spirin AS (1997) Cotranslational folding of globin. $J$ Biol Chem 272: 10646-10651

Komar AA, Lesnik T, Reiss C (1999) Synonymous codon substitutions affect ribosome traffic and protein folding during in vitro translation. FEBS Lett 462: 387-391

Kothe U, Rodnina MV (2006) Delayed release of inorganic phosphate from elongation factor Tu following GTP hydrolysis on the ribosome. Biochemistry 45: 12767-12774

Kothe U, Wieden HJ, Mohr D, Rodnina MV (2004) Interaction of helix D of elongation factor Tu with helices 4 and 5 of protein L7/12 on the ribosome. J Mol Biol 336: 1011-1021

Krab IM, Parmeggiani A (1999) Mutagenesis of three residues, isoleucine-60, threonine-61, and aspartic acid-80, implicated in the GTPase activity of Escherichia coli elongation factor Tu. Biochemistry 38: 13035-13041

Kramer EB, Farabaugh PJ (2007) The frequency of translational misreading errors in E. coli is largely determined by tRNA competition. RNA 13: 87-96

Krasheninnikov IA, Komar AA, Adzhubei IA (1991) Nonuniform size distribution of nascent globin peptides, evidence for pause localization sites, and a contranslational protein-folding model. J Protein Chem 10: 445-453

Krzycki JA (2005) The direct genetic encoding of pyrrolysine. Curr Opin Microbiol 8: 706-712 
Kudla G, Murray AW, Tollervey D, Plotkin JB (2009) Coding-sequence determinants of gene expression in Escherichia coli. Science 324: 255-258

Laemmli UK (1970) Cleavage of structural proteins during the assembly of the head of bacteriophage T4. Nature 227: 680-685

LaRiviere FJ, Wolfson AD, Uhlenbeck OC (2001) Uniform binding of aminoacyl-tRNAs to elongation factor Tu by thermodynamic compensation. Science 294: 165-168

Laurberg M, Asahara H, Korostelev A, Zhu J, Trakhanov S, Noller HF (2008) Structural basis for translation termination on the $70 \mathrm{~S}$ ribosome. Nature 454: $852-857$

Laursen BS, Sorensen HP, Mortensen KK, Sperling-Petersen HU (2005) Initiation of protein synthesis in bacteria. Microbiol Mol Biol Rev 69: 101-123

Ledoux S, Olejniczak M, Uhlenbeck OC (2009) A sequence element that tunes Escherichia coli tRNA(Ala)(GGC) to ensure accurate decoding. Nat Struct Mol Biol 16: 359-364

Ledoux S, Uhlenbeck OC (2008) Different aa-tRNAs are selected uniformly on the ribosome. Mol Cell 31: 114-123

Lee HH, Molla MN, Cantor CR, Collins JJ (2010) Bacterial charity work leads to population-wide resistance. Nature 467: 82-85

Lemke EA, Summerer D, Geierstanger BH, Brittain SM, Schultz PG (2007) Control of protein phosphorylation with a genetically encoded photocaged amino acid. Nat Chem Biol 3: 769-772

Lepthien S, Hoesl MG, Merkel L, Budisa N (2008) Azatryptophans endow proteins with intrinsic blue fluorescence. Proc Natl Acad Sci USA 105: 16095-16100

Li GW, Oh E, Weissman JS (2012) The anti-Shine-Dalgarno sequence drives translational pausing and codon choice in bacteria. Nature 484: 538-541

Liang ST, Xu YC, Dennis P, Bremer H (2000) mRNA composition and control of bacterial gene expression. $J$ Bacteriol 182: 3037-3044

Liljas A, Ehrenberg M, Aqvist J (2011) Comment on "The mechanism for activation of GTP hydrolysis on the ribosome". Science 333: 37; author reply 37

Ling J, Reynolds N, Ibba M (2009) Aminoacyl-tRNA synthesis and translational quality control. Anпи Rev Microbiol 63: 61-78

Liu C, Young AL, Starling-Windhof A, Bracher A, Saschenbrecker S, Rao BV, Rao KV, Berninghausen O, Mielke T, Hartl FU, Beckmann R, Hayer-Hartl M (2010) Coupled chaperone action in folding and assembly of hexadecameric Rubisco. Nature 463: 197-202

Louie A, Jurnak F (1985) Kinetic studies of Escherichia coli elongation factor Tu-guanosine 5'triphosphate-aminoacyl-tRNA complexes. Biochemistry 24: 6433-6439

Louie A, Ribeiro NS, Reid BR, Jurnak F (1984) Relative affinities of all Escherichia coli aminoacyl-tRNAs for elongation factor Tu-GTP. J Biol Chem 259: 5010-5016

Lu J, Deutsch C (2005a) Folding zones inside the ribosomal exit tunnel. Nat Struct Mol Biol 12: $1123-1129$ 
$\mathrm{Lu} \mathrm{J}$, Deutsch C (2005b) Secondary structure formation of a transmembrane segment in $\mathrm{Kv}$ channels. Biochemistry 44: 8230-8243

Lu J, Deutsch C (2008) Electrostatics in the ribosomal tunnel modulate chain elongation rates. $J$ Mol Biol 384: 73-86

Makeyev EV, Kolb VA, Spirin AS (1996) Enzymatic activity of the ribosome-bound nascent polypeptide. FEBS Lett 378: 166-170

Marshall RA, Aitken CE, Dorywalska M, Puglisi JD (2008) Translation at the single-molecule level. Annu Rev Biochem 77: 177-203

McClory SP, Leisring JM, Qin D, Fredrick K (2010) Missense suppressor mutations in 16S rRNA reveal the importance of helices $\mathrm{h} 8$ and h14 in aminoacyl-tRNA selection. RNA 16: 1925-1934

Milon P, Konevega AL, Gualerzi CO, Rodnina MV (2008) Kinetic checkpoint at a late step in translation initiation. Mol Cell 30: 712-720

Milon P, Konevega AL, Peske F, Fabbretti A, Gualerzi CO, Rodnina MV (2007) Transient kinetics, fluorescence, and FRET in studies of initiation of translation in bacteria. Methods Enzymol 430: $1-30$

Milon P, Maracci C, Filonava L, Gualerzi CO, Rodnina MV (2012) Real-time assembly landscape of bacterial 30S translation initiation complex. Nat Struct Mol Biol, in press

Milon P, Rodnina MV (2012) Kinetic control of translation initiation in bacteria. Crit Rev Biochem Mol Biol, in press

Mitarai N, Sneppen K, Pedersen S (2008) Ribosome collisions and translation efficiency: optimization by codon usage and mRNA destabilization. J Mol Biol 382: 236-245

Mittelstaet J, Konevega AL, Rodnina MV (2011) Distortion of tRNA upon near-cognate codon recognition on the ribosome. J Biol Chem 286: 8158-8164

Mohr D, Wintermeyer W, Rodnina MV (2002) GTPase activation of elongation factors Tu and G on the ribosome. Biochemistry 41: 12520-12528

Munro JB, Altman RB, Tung CS, Cate JH, Sanbonmatsu KY, Blanchard SC (2010) Spontaneous formation of the unlocked state of the ribosome is a multistep process. Proc Natl Acad Sci USA 107: $709-714$

Nakamura Y, Gojobori T, Ikemura T (2000) Codon usage tabulated from international DNA sequence databases: status for the year 2000. Nucleic Acids Res 28: 292

Nakatogawa H, Murakami A, Mori H, Ito K (2005) SecM facilitates translocase function of SecA by localizing its biosynthesis. Genes Dev 19: 436-444

Netzer WJ, Hartl FU (1997) Recombination of protein domains facilitated by co-translational folding in eukaryotes. Nature 388: $343-349$

Neumann H, Peak-Chew SY, Chin JW (2008) Genetically encoding N(epsilon)-acetyllysine in recombinant proteins. Nat Chem Biol 4: 232-234

Neumann H, Wang K, Davis L, Garcia-Alai M, Chin JW (2010) Encoding multiple unnatural amino acids via evolution of a quadruplet-decoding ribosome. Nature 464: 441-444 
Nicola AV, Chen W, Helenius A (1999) Co-translational folding of an alphavirus capsid protein in the cytosol of living cells. Nat Cell Biol 1: $341-345$

Nissen P, Hansen J, Ban N, Moore PB, Steitz TA (2000) The structural basis of ribosome activity in peptide bond synthesis. Science 289: 920-930

Nissen P, Kjeldgaard M, Thirup S, Polekhina G, Reshetnikova L, Clark BF, Nyborg J (1995) Crystal structure of the ternary complex of Phe-tRNAPhe, EF-Tu, and a GTP analog. Science 270: $1464-1472$

O'Brien EP, Hsu ST, Christodoulou J, Vendruscolo M, Dobson CM (2010) Transient tertiary structure formation within the ribosome exit port. J Am Chem Soc 132: 16928-16937

O'Connor M, Dahlberg AE (1993) Mutations at U2555, a tRNA-protected base in 23S rRNA, affect translational fidelity. Proc Natl Acad Sci USA 90: 9214-9218

O'Connor M, Dahlberg AE (1995) The involvement of two distinct regions of $23 \mathrm{~S}$ ribosomal RNA in tRNA selection. $J$ Mol Biol 254: 838-847

Ogle JM, Brodersen DE, Clemons WM, Jr., Tarry MJ, Carter AP, Ramakrishnan V (2001) Recognition of cognate transfer RNA by the 30S ribosomal subunit. Science 292: 897-902

Ogle JM, Murphy FV, Tarry MJ, Ramakrishnan V (2002) Selection of tRNA by the ribosome requires a transition from an open to a closed form. Cell 111: 721-732

Ott G, Faulhammer HG, Sprinzl M (1989) Interaction of elongation factor Tu from Escherichia coli with aminoacyl-tRNA carrying a fluorescent reporter group on the 3' terminus. Eur J Biochem 184: $345-352$

Ott G, Schiesswohl M, Kiesewetter S, Forster C, Arnold L, Erdmann VA, Sprinzl M (1990) Ternary complexes of Escherichia coli aminoacyl-tRNAs with the elongation factor Tu and GTP: thermodynamic and structural studies. Biochim Biophys Acta 1050: 222-225

Ozbudak EM, Thattai M, Kurtser I, Grossman AD, van Oudenaarden A (2002) Regulation of noise in the expression of a single gene. Nat Genet 31: 69-73

Pan D, Zhang CM, Kirillov S, Hou YM, Cooperman BS (2008) Perturbation of the tRNA tertiary core differentially affects specific steps of the elongation cycle. J Biol Chem 283: 18431-18440

Pape T, Wintermeyer W, Rodnina M (1999) Induced fit in initial selection and proofreading of aminoacyl-tRNA on the ribosome. EMBO J 18: 3800-3807

Pape T, Wintermeyer W, Rodnina MV (1998) Complete kinetic mechanism of elongation factor Tu-dependent binding of aminoacyl-tRNA to the A site of the E. coli ribosome. EMBO J 17: 74907497

Pape T, Wintermeyer W, Rodnina MV (2000) Conformational switch in the decoding region of 16S rRNA during aminoacyl-tRNA selection on the ribosome. Nat Struct Biol 7: 104-107

Park HS, Hohn MJ, Umehara T, Guo LT, Osborne EM, Benner J, Noren CJ, Rinehart J, Soll D (2011) Expanding the genetic code of Escherichia coli with phosphoserine. Science 333: 11511154

Parker J (1989) Errors and alternatives in reading the universal genetic code. Microbiol Rev 53: 273-298 
Pavlov MY, Watts RE, Tan Z, Cornish VW, Ehrenberg M, Forster AC (2009) Slow peptide bond formation by proline and other N-alkylamino acids in translation. Proc Natl Acad Sci USA 106: 5054

Peske F, Rodnina MV, Wintermeyer W (2005) Sequence of steps in ribosome recycling as defined by kinetic analysis. Mol Cell 18: 403-412

Peske F, Savelsbergh A, Katunin VI, Rodnina MV, Wintermeyer W (2004) Conformational changes of the small ribosomal subunit during elongation factor G-dependent tRNA-mRNA translocation. J Mol Biol 343: 1183-1194

Peters T, Jr., Davidson LK (1982) The biosynthesis of rat serum albumin. In vivo studies on the formation of the disulfide bonds. J Biol Chem 257: 8847-8853

Piepenburg O, Pape T, Pleiss JA, Wintermeyer W, Uhlenbeck OC, Rodnina MV (2000) Intact aminoacyl-tRNA is required to trigger GTP hydrolysis by elongation factor Tu on the ribosome. Biochemistry 39: 1734-1738

Plotkin JB, Kudla G (2011) Synonymous but not the same: the causes and consequences of codon bias. Nat Rev Genet 12: 32-42

Proshkin S, Rahmouni AR, Mironov A, Nudler E (2010) Cooperation between translating ribosomes and RNA polymerase in transcription elongation. Science 328: 504-508

Protzel A, Morris AJ (1974) Gel chromatographic analysis of nascent globin chains. Evidence of nonuniform size distribution. J Biol Chem 249: 4594-4600

Purvis IJ, Bettany AJ, Santiago TC, Coggins JR, Duncan K, Eason R, Brown AJ (1987) The efficiency of folding of some proteins is increased by controlled rates of translation in vivo. A hypothesis. J Mol Biol 193: 413-417

Qu X, Wen JD, Lancaster L, Noller HF, Bustamante C, Tinoco I, Jr. (2011) The ribosome uses two active mechanisms to unwind messenger RNA during translation. Nature 475: 118-121

Reynolds NM, Lazazzera BA, Ibba M (2010) Cellular mechanisms that control mistranslation. Nat Rev Microbiol 8: 849-856

Robertson JM, Wintermeyer W (1981) Effect of translocation on topology and conformation of anticodon and D loops of tRNA ${ }^{\text {Phe }}$. J Mol Biol 151: 57-79

Roder H, Colon W (1997) Kinetic role of early intermediates in protein folding. Curr Opin Struct Biol 7: 15-28

Rodnina MV, Fricke R, Kuhn L, Wintermeyer W (1995) Codon-dependent conformational change of elongation factor Tu preceding GTP hydrolysis on the ribosome. EMBO J 14: 2613-2619

Rodnina MV, Fricke R, Wintermeyer W (1994) Transient conformational states of aminoacyltRNA during ribosome binding catalyzed by elongation factor Tu. Biochemistry 33: 12267-12275

Rodnina MV, Gromadski KB, Kothe U, Wieden HJ (2005) Recognition and selection of tRNA in translation. FEBS Lett 579: 938-9342

Rodnina MV, Pape T, Fricke R, Kuhn L, Wintermeyer W (1996) Initial binding of the elongation factor Tu.GTP-aminoacyl-tRNA complex preceding codon recognition on the ribosome. J Biol Chem 271: 646-652 
Rodnina MV, Savelsbergh A, Katunin VI, Wintermeyer W (1997) Hydrolysis of GTP by elongation factor $\mathrm{G}$ drives tRNA movement on the ribosome. Nature 385: 37-41

Rodnina MV, Savelsbergh A, Matassova NB, Katunin VI, Semenkov YP, Wintermeyer W (1999) Thiostrepton inhibits the turnover but not the GTPase of elongation factor $\mathrm{G}$ on the ribosome. Proc Natl Acad Sci USA 96: 9586-9590

Rodnina MV, Wintermeyer W (1995) GTP consumption of elongation factor Tu during translation of heteropolymeric mRNAs. Proc Natl Acad Sci USA 92: 1945-1949

Rodnina MV, Wintermeyer W (2001) Fidelity of aminoacyl-tRNA selection on the ribosome: Kinetic and structural mechanisms. Annu Rev Biochem 70: 415-435

Rodnina MV, Wintermeyer W (2011) The ribosome as a molecular machine: the mechanism of tRNA-mRNA movement in translocation. Biochem Soc Trans 39: 658-662

Rodriguez EA, Lester HA, Dougherty DA (2006) In vivo incorporation of multiple unnatural amino acids through nonsense and frameshift suppression. Proc Natl Acad Sci USA 103: 86508655

Sanbonmatsu KY (2006) Alignment/misalignment hypothesis for tRNA selection by the ribosome. Biochimie 88: 1075-1089

Sanbonmatsu KY, Joseph S, Tung CS (2005) Simulating movement of tRNA into the ribosome during decoding. Proc Natl Acad Sci USA 102: 15854-15859

Sanderson LE, Uhlenbeck OC (2007) Directed mutagenesis identifies amino acid residues involved in elongation factor Tu binding to yeast Phe-tRNAPhe. J Mol Biol 368: 119-130

Savelsbergh A, Katunin VI, Mohr D, Peske F, Rodnina MV, Wintermeyer W (2003) An elongation factor G-induced ribosome rearrangement precedes tRNA-mRNA translocation. Mol Cell 11: $1517-1523$

Schägger H, von Jagow G (1987) Tricine-sodium dodecyl sulfate-polyacrylamide gel electrophoresis for the separation of proteins in the range from 1 to $100 \mathrm{kDa}$. Anal Biochem 166: 368-379

Schmeing TM, Ramakrishnan V (2009) What recent ribosome structures have revealed about the mechanism of translation. Nature 461: 1234-1242

Schmeing TM, Seila AC, Hansen JL, Freeborn B, Soukup JK, Scaringe SA, Strobel SA, Moore PB, Steitz TA (2002) A pre-translocational intermediate in protein synthesis observed in crystals of enzymatically active 50S subunits. Nat Struct Biol 9: 225-230

Schmeing TM, Voorhees RM, Kelley AC, Gao YG, Murphy FVt, Weir JR, Ramakrishnan V (2009) The crystal structure of the ribosome bound to EF-Tu and aminoacyl-tRNA. Science 326: 688-694

Schmeing TM, Voorhees RM, Kelley AC, Ramakrishnan V (2011) How mutations in tRNA distant from the anticodon affect the fidelity of decoding. Nat Struct Mol Biol 18: 432-436

Schrader JM, Chapman SJ, Uhlenbeck OC (2011) Tuning the affinity of aminoacyl-tRNA to elongation factor Tu for optimal decoding. Proc Natl Acad Sci USA 108: 5215-5220

Schubert HL, Phillips JD, Hill CP (2003) Structures along the catalytic pathway of PrmC/HemK, an N5-glutamine AdoMet-dependent methyltransferase. Biochemistry 42: 5592-5599 
Schuette J-C, Murphy FV, Kelly AC, Weir J, Giesebrecht J, Connell SR, Loerke J, Mielke J, Zhang W, Penczek P, Ramakrishnan V, Spahn CMT (2009) GTPase activation of elongation factor Tu by the ribosome during decoding. EMBO J 28: 619-620

Schultz DW, Yarus M (1994) tRNA structure and ribosomal function. I. tRNA nucleotide 27-43 mutations enhance first position wobble. J Mol Biol 235: 1381-1394

Sergiev PV, Lesnyak DV, Kiparisov SV, Burakovsky DE, Leonov AA, Bogdanov AA, Brimacombe R, Dontsova OA (2005) Function of the ribosomal E-site: a mutagenesis study. Nucleic Acids Res 33: 6048-6056

Sharma S, Chakraborty K, Muller BK, Astola N, Tang YC, Lamb DC, Hayer-Hartl M, Hartl FU (2008) Monitoring protein conformation along the pathway of chaperonin-assisted folding. Cell 133: $142-153$

Shaw JJ, Green R (2007) Two distinct components of release factor function uncovered by nucleophile partitioning analysis. Mol Cell 28: 458-467

Shine J, Dalgarno L (1974) The 3'-terminal sequence of Escherichia coli 16S ribosomal RNA: complementarity to nonsense triplets and ribosome binding sites. Proc Natl Acad Sci USA 71: $1342-1346$

Sievers A, Beringer M, Rodnina MV, Wolfenden R (2004) The ribosome as an entropy trap. Proc Natl Acad Sci USA 101: 7897-7901

Siller E, DeZwaan DC, Anderson JF, Freeman BC, Barral JM (2010) Slowing bacterial translation speed enhances eukaryotic protein folding efficiency. J Mol Biol 396: 1310-1318

Smith D, Yarus M (1989a) Transfer RNA structure and coding specificity. I. Evidence that a D-arm mutation reduces tRNA dissociation from the ribosome. J Mol Biol 206: 489-501

Smith D, Yarus M (1989b) Transfer RNA structure and coding specificity. II. A D-arm tertiary interaction that restricts coding range. J Mol Biol 206: 503-511

Sorensen MA, Kurland CG, Pedersen S (1989) Codon usage determines translation rate in Escherichia coli. J Mol Biol 207: 365-377

Sorensen MA, Pedersen S (1991) Absolute in vivo translation rates of individual codons in Escherichia coli. The two glutamic acid codons GAA and GAG are translated with a threefold difference in rate. $J$ Mol Biol 222: 265-280

Sprinzl M, Horn C, Brown M, Ioudovitch A, Steinberg S (1998) Compilation of tRNA sequences and sequences of tRNA genes. Nucleic Acids Res 26: 148-153

Stanzel M, Schön A, Sprinzl M (1994) Discrimination against misacylated tRNA by chloroplast elongation factor Tu. Eur J Biochem 219: 435-439

Starck SR, Qi X, Olsen BN, Roberts RW (2003) The puromycin route to assess stereo- and regiochemical constraints on peptide bond formation in eukaryotic ribosomes. J Am Chem Soc 125: 8090-8091

Stark H, Rodnina MV, Wieden HJ, Zemlin F, Wintermeyer W, van Heel M (2002) Ribosome interactions of aminoacyl-tRNA and elongation factor Tu in the codon-recognition complex. Nat Struct Biol 9: 849-854 
Steitz TA (2008) A structural understanding of the dynamic ribosome machine. Nat Rev Mol Cell Biol 9: 242-253

Studer SM, Joseph S (2006) Unfolding of mRNA secondary structure by the bacterial translation initiation complex. Mol Cell 22: 105-115

Taki M, Hohsaka T, Murakami H, Taira K, Sisido M (2002) Position-specific incorporation of a fluorophore-quencher pair into a single streptavidin through orthogonal four-base codon/anticodon pairs. J Am Chem Soc 124: 14586-14590

Takyar S, Hickerson RP, Noller HF (2005) mRNA helicase activity of the ribosome. Cell 120: 4958

Tang Y, Tirrell DA (2001) Biosynthesis of a highly stable coiled-coil protein containing hexafluoroleucine in an engineered bacterial host. J Am Chem Soc 123: 11089-11090

Tang Y, Wang P, Van Deventer JA, Link AJ, Tirrell DA (2009) Introduction of an aliphatic ketone into recombinant proteins in a bacterial strain that overexpresses an editing-impaired leucyl-tRNA synthetase. Chembiochem 10: 2188-2190

Tanrikulu IC, Schmitt E, Mechulam Y, Goddard WA, 3rd, Tirrell DA (2009) Discovery of Escherichia coli methionyl-tRNA synthetase mutants for efficient labeling of proteins with azidonorleucine in vivo. Proc Natl Acad Sci USA 106: 15285-15290

Thomas LK, Dix DB, Thompson RC (1988) Codon choice and gene expression: synonymous codons differ in their ability to direct aminoacylated-transfer RNA binding to ribosomes in vitro. Proc Natl Acad Sci USA 85: 4242-4246

Tomsic J, Vitali LA, Daviter T, Savelsbergh A, Spurio R, Striebeck P, Wintermeyer W, Rodnina MV, Gualerzi CO (2000) Late events of translation initiation in bacteria: a kinetic analysis. EMBO J 19: $2127-2136$

Vacher J, Buckingham RH (1979) Effect of photochemical crosslink S4U(8)-C(13) on suppressor activity of su+ tRNATrp from Escherichia coli. J Mol Biol 129: 287-294

Valle M, Sengupta J, Swami NK, Grassucci RA, Burkhardt N, Nierhaus KH, Agrawal RK, Frank J (2002) Cryo-EM reveals an active role for aminoacyl-tRNA in the accommodation process. $E M B O$ J 21: $3557-3567$

Valle M, Zavialov A, Li W, Stagg SM, Sengupta J, Nielsen RC, Nissen P, Harvey SC, Ehrenberg M, Frank J (2003) Incorporation of aminoacyl-tRNA into the ribosome as seen by cryo-electron microscopy. Nat Struct Biol 10: 899-906

Varenne S, Buc J, Lloubes R, Lazdunski C (1984) Translation is a non-uniform process. Effect of tRNA availability on the rate of elongation of nascent polypeptide chains. J Mol Biol 180: 549-576

Vetter IR, Wittinghofer A (2001) The guanine nucleotide-binding switch in three dimensions. Science 294: 1299-1304

Villa E, Sengupta J, Trabuco LG, Lebarron J, Baxter WT, Shaikh TR, Grassucci RA, Nissen P, Ehrenberg M, Schulten K, Frank J (2009) Ribosome-induced changes in elongation factor Tu conformation control GTP hydrolysis. Proc Natl Acad Sci USA 106: 1063-1068

Voorhees RM, Schmeing TM, Kelley AC, Ramakrishnan V (2010) The mechanism for activation of GTP hydrolysis on the ribosome. Science 330: 835-838 
Vorstenbosch E, Pape T, Rodnina MV, Kraal B, Wintermeyer W (1996) The G222D mutation in elongation factor $\mathrm{Tu}$ inhibits the codon-induced conformational changes leading to GTPase activation on the ribosome. EMBO J 15: 6766-6774

Voss NR, Gerstein M, Steitz TA, Moore PB (2006) The geometry of the ribosomal polypeptide exit tunnel. J Mol Biol 360: 893-906

Walter P, Blobel G (1981) Translocation of proteins across the endoplasmic reticulum III. Signal recognition protein (SRP) causes signal sequence-dependent and site-specific arrest of chain elongation that is released by microsomal membranes. J Cell Biol 91: 557-561

Wang K, Neumann H, Peak-Chew SY, Chin JW (2007) Evolved orthogonal ribosomes enhance the efficiency of synthetic genetic code expansion. Nat Biotechnol 25: 770-777

Wang L, Brock A, Herberich B, Schultz PG (2001) Expanding the genetic code of Escherichia coli. Science 292: 498-500

Weinreis SA, Ellis JP, Cavagnero S (2010) Dynamic fluorescence depolarization: a powerful tool to explore protein folding on the ribosome. Methods 52: 57-73

Weixlbaumer A, Jin H, Neubauer C, Voorhees RM, Petry S, Kelley AC, Ramakrishnan V (2008) Insights into translational termination from the structure of RF2 bound to the ribosome. Science 322: $953-956$

Wen JD, Lancaster L, Hodges C, Zeri AC, Yoshimura SH, Noller HF, Bustamante C, Tinoco I (2008) Following translation by single ribosomes one codon at a time. Nature 452: 598-603

Whitford PC, Geggier P, Altman RB, Blanchard SC, Onuchic JN, Sanbonmatsu KY (2010) Accommodation of aminoacyl-tRNA into the ribosome involves reversible excursions along multiple pathways. RNA 16: 1196-1204

Wieden HJ, Gromadski K, Rodnin D, Rodnina MV (2002) Mechanism of elongation factor (EF)Ts-catalyzed nucleotide exchange in EF-Tu. Contribution of contacts at the guanine base. J Biol Chem 277: 6032-6036

Wintermeyer W, Zachau HG (1979) Fluorescent derivatives of yeast tRNA ${ }^{\text {Phe }}$. Eur J Biochem 98: 465-475

Wohlgemuth I, Pohl C, Mittelstaet J, Konevega AL, Rodnina MV (2011) Evolutionary optimization of speed and accuracy of decoding on the ribosome. Philos Trans R Soc Lond B Biol Sci 366: 2979-2986

Wohlgemuth I, Pohl C, Rodnina MV (2010) Optimization of speed and accuracy of decoding in translation. EMBO J 29: 3701-3709

Wolin SL, Walter P (1988) Ribosome pausing and stacking during translation of a eukaryotic mRNA. EMBO J 7: 3559-3569

Woolhead CA, McCormick PJ, Johnson AE (2004) Nascent membrane and secretory proteins differ in FRET-detected folding far inside the ribosome and in their exposure to ribosomal proteins. Cell 116: 725-736

Wu N, Deiters A, Cropp TA, King D, Schultz PG (2004) A genetically encoded photocaged amino acid. J Am Chem Soc 126: 14306-14307

Xie J, Schultz PG (2005) An expanding genetic code. Methods 36: 227-238 
Yang Z, Shipman L, Zhang M, Anton BP, Roberts RJ, Cheng X (2004) Structural characterization and comparative phylogenetic analysis of Escherichia coli HemK, a protein (N5)-glutamine methyltransferase. J Mol Biol 340: 695-706

Yanofsky C (2004) The different roles of tryptophan transfer RNA in regulating trp operon expression in E. coli versus B. subtilis. Trends Genet : TIG 20: 367-374

Ye S, Zaitseva E, Caltabiano G, Schertler GF, Sakmar TP, Deupi X, Vogel R (2010) Tracking Gprotein-coupled receptor activation using genetically encoded infrared probes. Nature 464: 13861389

Yusupova G, Jenner L, Rees B, Moras D, Yusupov M (2006) Structural basis for messenger RNA movement on the ribosome. Nature 444: 391-394

Yusupova GZ, Yusupov MM, Cate JH, Noller HF (2001) The path of messenger RNA through the ribosome. Cell 106: 233-241

Zaher HS, Green R (2009a) Fidelity at the molecular level: Lessons from protein synthesis. Cell 136: $746-762$

Zaher HS, Green R (2009b) Quality control by the ribosome following peptide bond formation. Nature 457: 161-166

Zaher HS, Green R (2010) Kinetic basis for global loss of fidelity arising from mismatches in the P-site codon:anticodon helix. RNA 16: 1980-1989

Zaher HS, Green R (2011) A primary role for release factor 3 in quality control during translation elongation in Escherichia coli. Cell 147: 396-408

Zhang G, Hubalewska M, Ignatova Z (2009) Transient ribosomal attenuation coordinates protein synthesis and co-translational folding. Nat Struct Mol Biol 16: 274-280

Zhang G, Ignatova Z (2009) Generic algorithm to predict the speed of translational elongation: implications for protein biogenesis. PLoS One 4: e5036

Zouridis H, Hatzimanikatis V (2008) Effects of codon distributions and tRNA competition on protein translation. Biophys J 95: 1018-1033 


\section{ACKNOWLEDGEMENTS}

I would like to express my sincere thanks to Marina Rodnina for being a great supervisor of my $\mathrm{PhD}$ thesis. Her encouragement and enthusiasm for science helped me in all the phases of my work.

I am grateful to Wolfgang Wintermeyer for his helpful advices and constructive criticism.

I would also like to thank my thesis committee members Ralf Ficner and Holger Stark for their time and helpful suggestions during our meetings.

I would like to thank Andrey Konevega for introducing me to the world of tRNAs, his help, and for many advices during all the phases of my $\mathrm{PhD}$ thesis.

Furthermore, I would like to thank Anton Komar and Sujata Jha (Cleveland State University) as well as Harald Schwalbe and Florian Buhr (Goethe University, Frankfurt) for many stimulating discussions within the framework of our collaboration on co-translational protein folding.

I would like to thank Corinna Pohl, Ingo Wohlgemuth, and Wolf Holtkamp for sharing the time in the lab, many helpful discussions, and encouragement during times of frustration. Moreover, I would like to thank Wolf for our collaboration in joint projects.

Many thanks to Frank Peske for helpful suggestions and support with molecular cloning, Pohl Milon for organizing joint methods courses, Michael Thommen for help with the analysis of translation intermediates, Dmitry Burakovskiy for being a good bench neighbor, and Ekaterina Samatova for helpful discussions on protein folding.

Special thanks to Sandra Kappler, Christina Kothe, Olaf Geintzer, Tanja Wiles, and Theresia Uhlendorf for expert technical assistance and many preparations of ribosomes, tRNAs, and translation factors, and to Dimitra Papastavrou for help in organizational affairs.

Furthermore, I would like to thank all the other members of the Department of Physical Biochemistry and the Ribosome Dynamics Group for creating a nice and motivating atmosphere.

Most of all I am thankful to my parents and family for all their understanding and enduring support during my whole life. Especially, I would like to thank Kathrin for all her support, patience, and for just being there. 


\section{CURRICULUM VITAE}

\section{Personal data}

Name

Jörg Mittelstät

Date of Birth

$\operatorname{March} 23^{\text {rd }}, 1982$

Place of Birth

Oberhausen, Germany

\section{Scientific Education}

$04 / 2008-06 / 2012$

Ph.D. studies at the Department of Physical Biochemistry Max Planck Institute for Biophysical Chemistry, Göttingen

$10 / 2005-03 / 2008$

M.Sc. studies in Biochemistry and Molecular Cell Biology

University of Witten/Herdecke

$10 / 2002-09 / 2005$

B.Sc. studies in Water: Chemistry-Analytics-Microbiology

University of Duisburg-Essen

\section{School Education}

$1992-2001$

High school

Vestisches Gymnasium Kirchhellen, Germany

Degree: University Entrance Diploma (Abitur)

1988-1992

Elementary school

Grundschule Grafenwald, Bottrop, Germany 